\title{
Princípio dos grandes desvios para estados de Gibbs-equilíbrio sobre shifts enumeráveis à temperatura zero
}

\author{
Edgardo Enrique Pérez Reyes
}

TESE APRESENTADA

$\mathrm{AO}$

Instituto de Matemática e Estatística

DA

UNIVERSIDADE DE SÃO PAULO

PARA

OBTENÇÃO DO TÍTULO

$\mathrm{DE}$

DOUTOR EM CIÊNCIAS

\author{
Programa: Matemática Aplicada \\ Orientador: Prof. Dr. Rodrigo Bissacot \\ Co-orientador: Prof. Dr. Renaud Leplaideur
}

Durante o desenvolvimento deste trabalho o autor recebeu auxílio financeiro da CAPES/FAPESP

São Paulo, março de 2015 


\section{Princípio dos grandes desvios para estados de Gibbs-equilíbrio sobre shifts enumeráveis à temperatura zero}

Esta é a versão original da tese elaborada pelo candidato Edgardo Enrique Pérez Reyes, tal como submetida à Comissão Julgadora. 


\section{Princípio dos grandes desvios para estados de Gibbs-equilíbrio sobre shifts enumeráveis à temperatura zero}

Esta versão da tese contém as correções e alterações sugeridas pela Comissão Julgadora durante a defesa da versão original do trabalho, realizada em 13/03/2015. Uma cópia da versão original está disponível no Instituto de Matemática e Estatística da Universidade de São Paulo.

Comissão Julgadora:

- Prof. Dr. Renaud Leplaideur (co-orientador) - Université de Brest

- Prof. Dr. Artur Oscar Lopes - UFRGS

- Prof. Dr. Albert Fisher - IME-USP

- Prof. Dr. Ali Messaoudi -UNESP

- Prof. Dr. Ricardo dos Santos Freire Jr - IME-USP 


\section{Dedicatória}

Dedico este trabalho aos meus pais, Edgardo e Nicolasa, as minhas irmãs Maura e Odila e à minha noiva Diana. 


\section{Agradecimentos}

Primeiramente, agradeço aos meus orientadores Rodrigo Bissacot e Renaud Leplaideur, por suas orientações, por seus ensinamentos e amizade. Ao Rodrigo, por sua dedicação e disponibilidade durante estes três anos, e a paciência para com a finalização desta tese. Ao Renaud, por me convidar à França e sua hospitalidade lá, onde parte desta tese foi desenvolvida.

Aos meus pais, Edgardo e Nicolasa, por seu apoio incondicional sempre e por motivar a seus filhos a realizar sonhos e conquistas, as minhas irmãs, Maura e Odila pelo carinho e incentivo ao longo de todos estes anos.

À minha noiva Diana, pelo seu amor, paciência, compreensão e encorajamento, durante todo este período.

Ao Professor Jairo Mengue, por importantes discussões e sugestões ao longo deste trabalho.

Um agradecimento especial é devido também aos membros da banca examinadora pelas sugestões e correção do texto.

Aos amigos de casa e o instituto, em especial gostaria de agradecer a Hugo, Roger, Rodrigo e Deissy, muito obrigado pelo apoio e amizade.

Ao Professor Dumar Villa, por me incentivar a continuar os estudos de pós-graduação em matemática.

Ao programa de intercâmbio europeu BREUDS, pelo suporte financiero durante minha estadia na França.

A CAPES e a FAPESP pelo apoio financiero. 


\section{Resumo}

Seja $\Sigma_{A}(\mathbb{N})$ um shift enumerável topologicamente mixing com a propriedade BIP sobre o alfabeto $\mathbb{N}, f: \Sigma_{A}(\mathbb{N}) \rightarrow \mathbb{R}$ um potencial com variação somável e pressão topológica finita. Sob hipóteses adequadas provamos a existência de um princípio dos grandes desvios para a familia de estados de Gibbs $\left(\mu_{\beta}\right)_{\beta>0}$, onde cada $\mu_{\beta}$ é a medida de Gibbs associada ao potencial $\beta f$. Para fazer isso generalizamos alguns teoremas de Otimização Ergódica para shifts de Markov enumeráveis. Esse resultado generaliza o mesmo princípio no caso de um subshift topologicamente mixing sobre um alfabeto finito, previamente provado por A. Baraviera, A. Lopes e P. Thieullen.

Palavras-chave: Medida de Equilíbrio, medida de Gibbs, sub-ação, medida maximizante, Formalismo Termodinâmico, grandes desvios. 


\section{Abstract}

Let $\Sigma_{A}(\mathbb{N})$ be a topologically mixing countable Markov shift with the BIP property over the alphabet $\mathbb{N}$ and a potential $f: \Sigma_{A}(\mathbb{N}) \rightarrow \mathbb{R}$ with summable variation and finite pressure. Under suitable hypotheses, we prove the existence of a large deviation principle for the family of Gibbs states $\left(\mu_{\beta}\right)_{\beta>0}$ where each $\mu_{\beta}$ is the Gibbs measure associated to the potential $\beta f$. For do this we generalize some theorems from finite to countable Markov shifts in Ergodic Optimization. This result generalizes the same principle in the case of topologically mixing subshifts over a finite alphabet previously proved by A. Baraviera, A. Lopes and P. Thieullen.

Keywords: equilibrium measure, Gibbs measure, sub-action, maximizing measure, Thermodynamic Formalism, large deviations. 


\section{Sumário}

Lista de Símbolos

Lista de Figuras $\quad$ xiii

1 Introdução $\quad 1$

2 Preliminares $\quad 5$

2.1 Shifts de Markov sobre alfabetos enumeráveis . . . . . . . . . . . . . . . 5

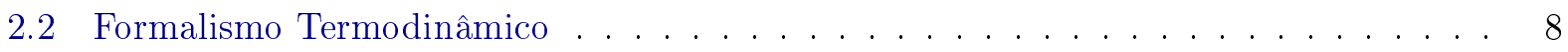

2.3 Temperatura Zero . . . . . . . . . . . . . . . . . . . . . . . 19

3 Otimização Ergódica $\quad 23$

3.1 Existência de maximizantes e sub-ações . . . . . . . . . . . . . . . . . . . 24

3.2 Barreira de Peierls e Potencial de Mañé . . . . . . . . . . . . . . . . . . . . 32

4 Princípio dos Grandes Desvios à Temperatura Zero 49

4.1 Princípio dos Grandes Desvios . . . . . . . . . . . . . . . . . . . . . . 49

4.2 Unicidade da medida maximizante . . . . . . . . . . . . . . 61

4.3 Pesquisas futuras . . . . . . . . . . . . . . . . . . . . 6 64

A Espaços de Potenciais:caso não-compacto $\quad 65$

B Grandes Desvios na Probabilidade $\quad 69$

$\begin{array}{ll}\text { Referências Bibliográficas } & 71\end{array}$ 


\section{Lista de Símbolos}

$\begin{array}{ll}\beta & \text { Inverso da temperatura } \\ \mu_{\beta} & \text { Medida de equilíbrio } \\ f & \text { Potencial com variação somável } \\ {\left[a_{0} \ldots a_{n-1}\right]} & \text { Cilindro de tamanho } n \\ \mathbb{A} & \text { Matriz de transição } \\ \Sigma_{A} & \text { Espaço de sequências } \\ \Sigma_{A}(\mathbb{N}) & \text { Espaço de seqüências permitidas } \\ H_{f} & \text { Constante de Hölder } \\ K_{0} & \text { Constante primitiva } \\ P(f) & \text { Pressão topológica } \\ h(\mu) & \text { Entropia métrica da medida } \mu \\ I & \text { Função taxa } \\ C^{0} & \text { Espaço das funções contínuas assumindo valores reais } \\ \mathcal{M}_{\sigma}\left(\Sigma_{A}(\mathbb{N})\right) & \text { Conjunto de medidas Borel invariantes para o mapa shift } \\ m(f) & \text { Valor maximal } \\ S_{n} f & \text { n-ésima soma de Birkhoff } \\ \sigma & \text { Dinâmica do shift } \\ S_{f}(x, y) & \text { Potencial de Mañé } \\ h_{f}(x, y) & \text { Barreira de Peierls } \\ \Omega_{(f, \sigma)} & \text { Conjunto de Aubry } \\ \operatorname{Var}_{(f)} & \text { Variação de } f \\ \operatorname{Var}_{n}(f) & n \text {-variação de } f \\ M_{f} \phi & \text { Operador de Lax-Oleinik } \\ L_{f}(g) & \text { Operador de Ruelle-Perron-Frobenius sobre o espaço das funções } \\ C_{b} & \text { Eontínuas limitadas } \\ & \text { Espaço das funções contínuas e limitadas de valor real } \\ & \end{array}$




\section{Lista de Figuras}

2.1 A sequência $x$ e $y$ coincidem do digito 0 até o digito $n-1$, e depois se dividem. . . 6

2.2 Exemplo de um shift com a propriedade BIP. . . . . . . . . . . . . . . . . . . . 7

2.3 Exemplo de um Renewal shift. . . . . . . . . . . . . . . . . . . . . . . . 8

3.1 Distancia de um ponto ao Aubry. . . . . . . . . . . . . . . . . . . . . 34 


\section{Capítulo 1}

\section{Introdução}

O trabalho nesta tese refere-se à Teoria Ergódica para sistemas dinâmicos simbólicos. Teoria Ergódica é a area da matemática que estuda sistemas dinâmicos do ponto de vista do comportamento estatístico de órbitas de uma transformação.

Teoria Ergódica tem suas origens na Mecânica Estatística e no estudo do comportamento a longo prazo de sistemas com grande número de partículas. Em tais sistemas, a descrição exata do comportamento de cada partícula pode ser inviável, mas através dos teoremas ergódicos podemos ser capazes de entender o comportamento a longo prazo de pontos genéricos e conectar o comportamento macroscópico do sistema com leis microscópicas governando partículas individuais.

Depois dos livros fundamentais de R. Bowen [Bow08] e R. Ruelle [Rue78] uma boa quantidade de literatura foi produzida por dinamicistas, probabilistas e físico-matemáticos interessados em resultados rigorosos inspirados em modelos da Mecânica Estatística. Esta teoria é conhecida atualmente como Formalismo Termodinâmico. Um dos problemas centrais na Mecânica Estatística e no Formalismo Termodinâmico é descrever a familia de estados de Gibbs para um potencial fixo. Muitos resultados desta área estão concentrados no caso unidimensional onde o espaço de estados é um conjunto finito $S$ e $\Omega=S^{\mathbb{Z}}$ (ou $S^{\mathbb{N}}$ ) é o espaço de configurações.

Nos últimos 15 anos houve intensa pesquisa na direção de estender a teoria unidimensional do caso compacto para o não compacto onde consideramos modelos com espaço de configurações $S^{\mathbb{Z}}$ (ou $S^{\mathbb{N}}$ ) e $S=\mathbb{N}$. Muitos dos resultados para modelos onde o espaço de estados é um conjunto finito já possuem uma formulação equivalente no caso de um espaço não compacto. Estes resultados foram obtidos principalmente por R. D. Mauldin e M. Urbański [MU03] e também por O. Sarig [Sar99, Sar03]. Os avanços de Sarig foram recentemente catalogados num survey escrito por Y. Pesin [Pes14].

Diferentemente do caso multidimensional, o caso de shifts de Markov unidimensionais e transitivos tem no máximo um estado de equilíbrio [Sar09, BS03] quando o potencial tem variação somável (estendido ao caso de potenciais Walters recentemente [Dao12]). Isto significa que as únicas possíveis transições de fase em relação ao comportamento do conjunto de medidas de equilíbrio para esta classe de sistemas é a transição entre existência e ausência da medida de equilíbrio quando consideramos diferentes $\beta$ 's. O. Sarig provou que o conjunto de $\beta$ 's positivos em que temos transição de fase pode ter medida de Lebesgue positiva [Sar01, Sar00], mesmo no caso onde o potencial depende de um número finito de coordenadas.

Otimização Ergódica é o estudo de medidas invariantes que maximizam ou minimizam a integral de uma função particular. Usando o Teorema Ergódico de Birkhoff este problema é equivalente a encontrar os pontos que maximizam o crescimento das somas ergódicas de $f$ (veja [Jen06]). Usando resultados de O. Sarig [Sar01], G. Iommi encontrou uma conexão entre transição de fase e Otimi- 
zação Ergódica, provando que a ausência de transições de fase em Renewal shifts é equivalente a existência de medidas maximizantes [Iom07].

Além de transição de fase, temos outras conexões entre Otimização Ergódica e Formalismo Termodinâmico. Os pontos de acumulação na topologia fraca* à temperatura zero de estados de equilíbrios são medidas maximizantes [BLL13, CLT01, Jen06, JMU05], sub-ações (um objeto usado para descrever o suporte de medidas maximizantes) podem ser construídas através de pontos de acumulação de $\frac{1}{\beta} \log h_{\beta}$ quando $\beta$ vai para o infinito, aqui $h_{\beta}$ é autofunção do operador de Ruelle [CLT01].

O Jenkinson, R.D Mauldin e M. Urbański mostraram em [JMU06] que para um potencial $f$ com variação somável, sobre um shift finitamente primitivo, a familia de estados Gibbs $\left(\mu_{\beta}\right)_{\beta}$ tem pelo menos um ponto de acumulação na topologia fraca* quando $\beta \rightarrow \infty$ e qualquer ponto de acumulação $\mu$ é uma medida maximizante; mais do que isso, I. Morris provou em [Mor] que qualquer ponto de acumulação de $\left(\mu_{\beta}\right)_{\beta}$ tem entropia maximal entre as medidas maximizantes.

O principal resultado desta tese é mostrar que a familia de estados de equilíbrio-Gibbs $\left(\mu_{\beta f}\right)_{\beta}$ satisfaz um princípio dos grandes desvios. Este resultado generaliza o trabalho de A. Baraviera, A. Lopes e P. Thieullen [BLT06] de shifts finitos para shifts enumeráveis. Para atingir dito objetivo, vamos assumir algumas hipóteses sobre o potencial e sobre nosso espaço shift. De fato, a prova apresentada utiliza o fato da medida de equilíbrio ser Gibbs, o que nos obriga a assumir que o shift tem a propriedade BIP (ver [Sar03]). É consenso entre especialistas em Formalismo Termodinâmico que o comportamento de shifts BIP é similar ao de shifts compactos [IJ13]. Nosso trabalho é mais um resultado que confirma esta expectativa.

Além disso, assumiremos que existe uma única medida maximizante para o potencial $f$. Essa condição é genérica no caso compacto em diversos espaços de potenciais, [Bou01, CLT01, BLL13, Jen06, QS12] e densa no caso não compacto [DUZ07]. Dita suposição sobre a medida maximizante nos permitirá garantir, por exemplo, que o limite quando $\beta \rightarrow \infty$ de $\mu_{\beta f}$ existe e, ademais, garantimos também que duas sub-ações calibradas são iguais módulo uma constante. Esse fato é usado para definir a função taxa do princípio dos grandes desvios.

O principal resultado desta tese é o seguinte teorema:

Teorema 1. Sejam $\left(\Sigma_{A}(\mathbb{N}), \sigma\right)$ um shift finitamente primitivo e $f: \Sigma_{A}(\mathbb{N}) \rightarrow \mathbb{R}$ com variação somável e satisfazendo $P(f)<\infty$, além disso assuma que $f$ admite uma única medida maximizante $\mu$. Para cada $\beta$, denotamos $\mu_{\beta}$, a única medida de equilíbrio associada a $\beta f$. Então $\left(\mu_{\beta}\right)_{\beta}$ satisfaz um Princípio dos Grandes Desvios em cilindros, isto é, para qualquer cilindro $C=\left[x_{0} \ldots x_{n-1}\right]$,

$$
\lim _{\beta \rightarrow \infty} \frac{1}{\beta} \log \mu_{\beta}(C)=-\inf _{x \in C} I(x)
$$

onde

$$
I(x)=\sum_{j=0}^{\infty}(V \circ \sigma-V-f+m(f)) \circ \sigma^{j}(x), \quad m(f)=\int f d \mu
$$

onde $V$ é uma sub-ação calibrada para $f$.

Nosso trabalho está organizado da seguinte maneira:

O capítulo 1 introduz conceitos básicos de Dinâmica Simbólica, Otimização Ergódica e Formalismo Termodinâmico, conceitos usados ao longo de nosso estudo.

No capítulo 2 estendemos para o caso de shifts enumeráveis as principais propriedades do Potencial de Mañé e da Barreira de Peierls já conhecidas para o caso de um shift compacto. Mostramos a 
existência de uma sub-ação calibrada limitada para o caso de um shift enumerável. Apresentamos uma nova prova da existência de medidas maximizantes para shifts finitamente primitivos

O capítulo 3 mostramos que a família de estados Gibbs $\left(\mu_{\beta}\right)_{\beta}$ satisfaz um princípio dos grandes desvios. Além disso, expõe-se no apêndice um compêndio de teoremas com alguns resultados sobre espaços de potenciais para shifts não compactos. 


\section{Capítulo 2}

\section{Preliminares}

Nesse capítulo revisitamos definições e resultados do Formalismo Termodinâmico em shifts com um número enumerável de símbolos que serão usados em toda tese.

\subsection{Shifts de Markov sobre alfabetos enumeráveis}

Seja $\mathbb{N}$ o conjunto de inteiros não negativos, o qual consideraremos como nosso alfabeto no decorrer deste trabalho, $\Sigma(\mathbb{N})$ o conjunto de sequências de elementos em $\mathbb{N}$. Dada uma matriz infinita $A: \mathbb{N} \times \mathbb{N} \rightarrow\{0,1\}$, denotamos por $\Sigma_{A}(\mathbb{N})$ o conjunto $\Sigma(\mathbb{N})$ de sequências permitidas, isto é:

$$
\Sigma_{A}(\mathbb{N}):=\left\{x \in \Sigma(\mathbb{N}): A\left(x_{i}, x_{i+1}\right)=1, \forall i \geq 0\right\} .
$$

Dado um inteiro não negativo $I$, denote por

$$
\Sigma_{A}(I)=\left\{x=\left(x_{0}, x_{1}, \ldots\right) \in\{0, \ldots, I\}^{\mathbb{N}}: A\left(x_{j}, x_{j+1}\right)=1\right\} .
$$

Se $w=\left(w_{0}, \ldots, w_{k}\right), \operatorname{com} w_{i} \in \mathbb{N}$ para $1 \leq i \leq k$, definimos em $\Sigma_{A}(\mathbb{N})$ o cilindro de comprimento $k$ pelo conjunto

$$
[w]:=\left\{x \in \Sigma_{A}(\mathbb{N}): x_{i}=w_{i} \text { para } 0 \leq i \leq k\right\} .
$$

Uma palavra $x$ é uma concatenação de símbolos de $\mathbb{N}$. Uma palavra chama-se $A$-permitida ou simplesmente permitida se o cilindro $[x]$ é não vazio. A dinâmica está dada por o mapa shift $\sigma: \Sigma_{A}(\mathbb{N}) \rightarrow \Sigma_{A}(\mathbb{N})$, definido por $\sigma\left(x_{0}, x_{1}, x_{2}, \ldots\right)=\left(x_{1}, x_{2}, \ldots\right)$. Denotamos por $\mathcal{M}_{\sigma}\left(\Sigma_{A}(\mathbb{N})\right)$ o conjunto de medidas de probabilidade, invariantes para $\sigma$, definidas sobre os Borelianos de $\Sigma_{A}(\mathbb{N})$.

Colocamos $t(x, y)=\inf \left(\left\{k: x_{k} \neq y_{k}\right\} \cup\{\infty\}\right)$. Fixado $r \in(0,1)$ e colocando $d(x, y)=d_{r}(x, y)=$ $r^{t(x, y)}$. Esta é uma métrica sobre $\Sigma_{A}(\mathbb{N})$ e a topologia gerada por esta métrica tem os cilindros como base. O mapa $\sigma$ é contínuo com respeito a esta topologia. Podemos representar esta distancia graficamente como aparece na figura 2.1 .

Equipamos $\mathcal{M}_{\sigma}\left(\Sigma_{A}(\mathbb{N})\right)$ com a seguinte topologia: dizemos que $\left(\mu_{n}\right)_{n \geq 1}$ converge na topologia fraca* para alguma $\mu$ se, somente se, $\int f d \mu_{n} \rightarrow \int f d \mu$ para cada função contínua e limitada $f: \Sigma_{A}(\mathbb{N}) \rightarrow \mathbb{R}$. Em nosso caso, uma condição equivalente é que $\mu_{n}(C) \rightarrow \mu(C)$ para todos os cilindros $C . C^{0}\left(\Sigma_{A}(\mathbb{N})\right)$ indica o espaço de funções contínuas assumindo valores reais sobre $\Sigma_{A}(\mathbb{N})$, equipada com a topologia de convergência uniforme sobre conjuntos compactos. (veja o apêndice para mais detalhes sobre esta convergência) Dada $f \in C^{0}\left(\Sigma_{A}(\mathbb{N})\right)$, definimos $S_{n} f=\sum_{j=0}^{n-1} f \circ \sigma^{j}$ e $S_{0} f=0$.

Fixada uma função $f: \Sigma_{A}(\mathbb{N}) \rightarrow \mathbb{R}$, definimos a $n$-variação de $f$ como

$$
\operatorname{Var}_{n} f:=\sup \left\{|f(x)-f(y)| ; x_{0}=y_{0}, \ldots, x_{n-1}=y_{n-1}\right\} .
$$

Definição 1. O potencial $f: \Sigma_{A}(\mathbb{N}) \rightarrow \mathbb{R}$ chama-se localmente Hölder contínuo quando existe uma 


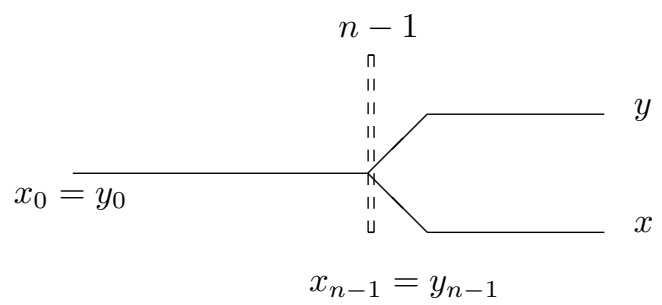

Figura 2.1: A sequência $x$ e y coincidem do digito 0 até o digito $n-1$, e depois se dividem.

constate $H_{f}>0$ tal que, para todo inteiro $k \geq 1$, temos que

$$
\operatorname{Var}_{k}(f):=\sup _{x, y \in \Sigma_{A}(\mathbb{N}), d(x, y) \leq r^{k}}[f(x)-f(y)] \leq H_{f} r^{k} .
$$

Dita condição de regularidade significa que a $k$-ésima variação $\operatorname{Var}_{k}(f)$ decai exponencialmente a zero quando $k \rightarrow \infty$. Dizemos que $f: \Sigma_{A}(\mathbb{N}) \rightarrow \mathbb{R}$ tem variação somável se

$$
\operatorname{Var}(f):=\sum_{k=1}^{\infty} \operatorname{Var}_{k}(f)<\infty
$$

Claramente a condição de $f$ ser localmente Hölder contínua é mais forte que variação somável. Cada uma destas condições implicam que $f$ é uniformemente contínua. No entanto elas não implicam que $f$ seja limitada, pois $\operatorname{Var}_{0}(f)=\sup _{x, y \in \Sigma_{A}(\mathbb{N})}|f(x)-f(y)|$ não está incluída em (2.1) ou em (2.2).

Definição 2. A função $f: \Sigma_{A}(\mathbb{N}) \rightarrow \mathbb{R}$ satisfaz a condição de Walters se $\lim _{k \rightarrow \infty} \sup _{n \geq 1} \operatorname{Var}_{n+k} S_{n} f=0$ e para cada $k \geq 1, \sup _{n \geq 1} \operatorname{Var}_{n+k} S_{n}(f)<\infty$.

A condição acima de regularidade foi introduzida por P. Walters em 1978 [Wal78] no contexto do Formalismo Termodinâmico. A definição original foi feita para shifts compactos, e não incluia a condição $\operatorname{Var}_{n+k} S_{n} f<\infty \forall k \geq 1, n \geq 1$. No caso compacto a finitude de $\operatorname{Var}_{n+k} S_{n} f \forall k \geq 1, n \geq 1$ segue da condição $\lim _{k \rightarrow \infty} \sup _{n \geq 1} \operatorname{Var}_{n+k} S_{n} f=0$, pois esta condição implica que $f$ é contínua e portanto limitada.

O seguinte lema mostra que a condição de Walters é mais fraca que a condição de variação somável ou localmente Hölder contínuo.

Lema 1. Seja $f: \Sigma_{A}(\mathbb{N}) \rightarrow \mathbb{R}$ com variação somável. Para todo $n \geq 1$ e qualquer palavra admissível $a=\left(a_{0}, \ldots, a_{n-1}\right)$ de comprimento $n$, se $x, y \in \sigma\left[a_{n-1}\right]$ e $x_{0}=y_{0}, \ldots, x_{m-1}=y_{m-1}$, então $\left|S_{n} f(a x)-S_{n} f(a y)\right| \leq \sum_{k=m+1}^{\infty} \operatorname{Var}_{k}(f)$.

Demonstração.

$$
\begin{aligned}
\left|S_{n} f(a x)-S_{n} f(a y)\right| & \leq \operatorname{Var}_{n+m}(f)+\operatorname{Var}_{n+m-1}(f)+\cdots+\operatorname{Var}_{m+1}(f) \\
& \leq \sum_{k=m+1}^{\infty} \operatorname{Var}_{k}(f) .
\end{aligned}
$$

Definição 3. Dizemos que $\left(\Sigma_{A}(\mathbb{N}), \sigma\right)$ é primitivo quando, existe $K_{0} \in \mathbb{N}$ e um sub-alfabeto $\mathbb{F} \subseteq \mathbb{N}$ tal que qualquer par de símbolos permitidos do alfabeto $\mathbb{N}$ podem ser conectados por uma palavra com exatamente $K_{0}$ símbolos em $\mathbb{F}$. Diremos que $\left(\Sigma_{A}(\mathbb{N}), \sigma\right)$ é finitamente primitivo quando $\mathbb{F}$ é finito.

Quando $\left(\Sigma_{A}(\mathbb{N}), \sigma\right)$ é finitamente primitivo, definimos

$$
I_{\mathbb{F}}:=\max \{i: i \in \mathbb{F}\} .
$$




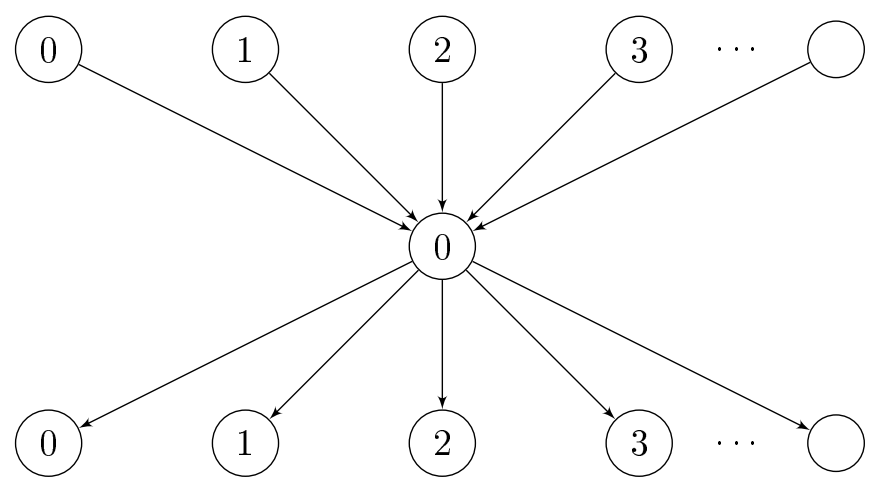

Figura 2.2: Exemplo de um shift com a propriedade BIP.

Definição 4. $\left(\Sigma_{A}(\mathbb{N}), \sigma\right)$ é dito topologicamente mixing quando

$$
\forall a, b \in \mathbb{N} \exists N_{a b} \forall n>N_{a b},[a] \cap \sigma^{-n}[b] \neq \emptyset .
$$

Definição 5. Dizemos que $\left(\Sigma_{A}(\mathbb{N}), \sigma\right)$ satisfaz a propriedade de "big image" e "preimage" $(B I P)$ se, e somente se, existe um conjunto finito $B=\left\{b_{1}, \ldots, b_{m}\right\}$ de elementos de $\mathbb{N}$ tal que para qualquer $a \in \mathbb{N}$, permitido, existem $i, j \in\{1, \ldots, m\}$ com $A\left(b_{i}, a\right)=A\left(a, b_{j}\right)=1$.

Os seguintes dois lemas mostram que as condições finitamente primitivo e BIP são equivalentes em shifts topologicamente mixing:

Lema 2. Seja $\left(\Sigma_{A}(\mathbb{N}), \sigma\right)$ um shift topologicamente mixing com a propriedade BIP, então ele é finitamente primitivo.

Demonstração. Como $\left(\Sigma_{A}(\mathbb{N}), \sigma\right)$ tem a propriedade BIP, então existe um conjunto finito, digamos $B=\left\{b_{1}, b_{2}, \ldots, b_{l}\right\}$ tal que para qualquer $a \in \mathbb{N}$ existem $i, j \in\{1,2, \ldots, l\}$ tal que $b_{i} a b_{j}$ é uma palavra permitida. Como $\left(\Sigma_{A}(\mathbb{N}), \sigma\right)$ é topologicamente mixing, então existem palavras de comprimento finito, $w_{b_{i} b_{j}}$ conectando $b_{i}$ e $b_{j}$, para todo $i, j \in\{1, \ldots, l\}$, isto é $b_{i} w_{b_{i} b_{j}} b_{j}$ são palavras permitidas para todo $i, j \in\{1, \ldots, l\}$.

Seja $N_{0}:=\max \left\{\left|w_{b_{i} b_{j}}\right|: 1 \leq i, j \leq l\right\}$, então por hipóteses sabemos que existem palavras $w_{b_{i} b_{j}}^{\prime}$ com comprimento igual a $N_{0}$ conectando $b_{i}$ e $b_{j}$, para todo $i, j \in\{1, \ldots, l\}$, isto é $b_{i} w_{b_{i} b_{j}}^{\prime} b_{j}$ são palavras permitidas para todo $i, j \in\{1, \ldots, l\}$.

Concluímos a prova definindo para $i, j \in\{1, \ldots, l\}$ o alfabeto finito $\mathbb{F}:=\left\{b_{1}, b_{2}, \ldots, b_{l}, w_{b_{i} b_{j}}^{\prime}\right\}$, onde $w_{b_{i} b_{j}}^{\prime}$ são todas as palavras de comprimento $N_{0}$ conectando $b_{i}$ e $b_{j}$, e seja $K_{0}:=N_{0}+2$. Desta forma temos que para qualquer par $x, y \in \mathbb{N}$, encontramos $l_{1}, l_{2}, \ldots, l_{K_{0}} \in \mathbb{F}$ cumprindo

$$
A\left(x, l_{1}\right) A\left(l_{1}, l_{2}\right) \cdots A\left(l_{K_{0}}, y\right)=1 .
$$

Lema 3. Seja $\left(\Sigma_{A}(\mathbb{N}), \sigma\right)$ um shift finitamente primitivo, então ele tem a propriedade BIP.

Demonstração. Seja $\mathbb{F} \subset \mathbb{N}$ finito e seja $K_{0} \geq 0$ um inteiro, tal que para qualquer par de símbolos $x, y$, podemos achar $l_{1}, \ldots, l_{K_{0}} \in \mathbb{F}$ tal que

$$
A\left(x, l_{1}\right) \cdots A\left(l_{K_{0}}, y\right)=1 .
$$

Agora definimos $B:=\left\{l_{1}, \ldots, l_{K_{0}}\right\}$, então para qualquer $a \in \mathbb{N}$ existem $l_{1}$ e $l_{K_{0}}$ tais que

$$
A\left(l_{k_{0}}, a\right) A\left(a, l_{1}\right)=1 .
$$




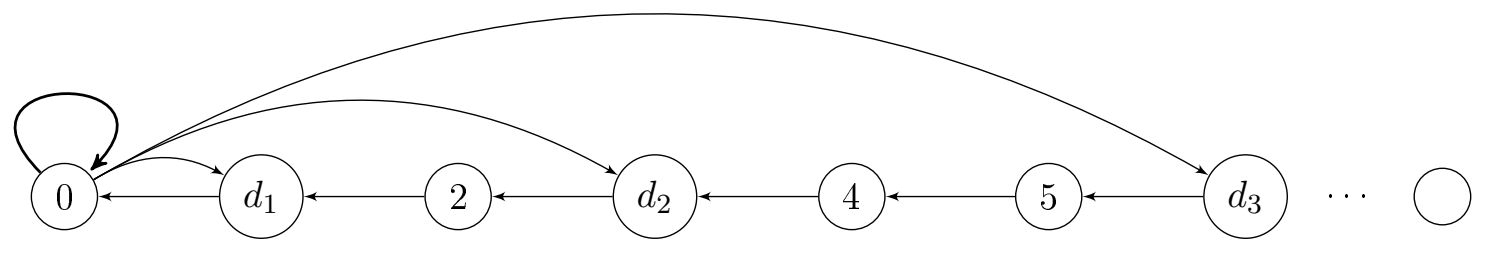

Figura 2.3: Exemplo de um Renewal shift.

Um exemplo importante de um shift que não tem a propriedade BIP e que é topologicamente mixing é o Renewal shift (veja [Iom07]).

Definição 6 (Renewal Shift). Dizemos que $\left(\Sigma_{A}(\mathbb{N}), \sigma\right)$ é um shift Renewal se para cada $n$ existe no máximo uma palavra permitida $x_{0} \ldots x_{n}$ de comprimento $n+1$ tais que $A\left(x_{n}, 0\right)=1$ e $x_{i}=0$ se e somente se $i=0$ (neste caso 0 é o renewal vértice).

O seguinte shift é um exemplo de um Renewal shift:

Exemplo 1. Seja $\left(d_{i}\right)_{i \geq 1}$ uma sequência crescente de inteiros positivos. Considere a matriz de transição $A=\left(a_{i j}\right)_{\mathbb{N} \times \mathbb{N}}$ com entradas $a_{00}, a_{i+1, i}, a_{0, d_{i}}$ equal a 1 e o resto de entradas igual a zero.

Usando os resultados de Sarig [Sar01], G. Iommi [Iom07] mostrou que no Renewal shift a existência de medidas maximizantes para um potencial localmente Hölder contínuo equivale à analiticidade da pressão para todo $\beta>0$. Voltaremos neste exemplo no futuro.

\subsection{Formalismo Termodinâmico}

Fixado um potencial $f: \Sigma_{A} \rightarrow \mathbb{R}$ com alguma regularidade (localmente Hölder contínuo, variação somável, Walters), alguns dos objetos de interesse quando fazemos Formalismo Termodinâmico são: existencia e número de medidas de Gibbs/equilíbrio para cada função $\beta f$ da família a um parâmetro $(\beta f)_{\beta>0}$, diferenciabilidade ou não da pressão $P(\beta f)$ e, a descrição das medidas de equilíbrio $\left(\mu_{\beta}\right)_{\beta}$ (quando existem) e eventualmente estudar o comportamento destes objetos quando a temperatura vai à zero, ou seja, $\beta \rightarrow \infty$. Uma das grandes diferenças entre o Formalismo Termodinâmico para shifts topologicamente mixing com um número finito de símbolos e o nosso contexto, é que a dinâmica do mapa shift definida pela matriz $A$ tem um papel fundamental no comportamento destes objetos. Atualmente sabemos que os shifts finitamente primitivos possuem muitas características em comum com os shifts compactos. Isso será fundamental em nossos principais resultados. A título de exemplo: se $f$ tem variação somável então a pressão $P(\beta f)$ é analítica em $\beta$ e, para cada $\beta>0$, a única medida de equilíbrio $\mu_{\beta}$ é Gibbsiana. Como nosso enfoque é a ligação entre o Formalismo Termodinâmico e Otimização Ergódica, vamos recordar rapidamente definições e alguns resultados de maneira que possamos tomar o limite $\beta \rightarrow \infty$.

Listaremos agora alguns dos principais resultados obtidos por O. Sarig e R. D. Mauldin e M. Urbański para o desenvolvimento do Formalismo Termodinâmico em shifts enumeráveis. Vários são generalizações de trabalhos de D. Vere-Jones [VJ67].

Definição 7. Seja $\left(\Sigma_{A}(\mathbb{N}), \sigma\right)$ um shift topologicamente mixing. Seja $g: \Sigma_{A}(\mathbb{N}) \rightarrow \mathbb{R}$ Walters. A pressão de Gurevich de gé definida por:

$$
P(g):=\lim _{n \rightarrow \infty} \frac{1}{n} \log \left(\sum_{\sigma^{n}(x)=x} \exp \left(S_{n} g(x)\right) 1_{[a]}(x)\right)
$$

donde a é um elemento permitido de $\mathbb{N}$. 
Observe que $P(g)$ pode ser infinita. Não é difícil mostrar que a definição independe do símbolo $a$ pois para qualquer dois símbolos $a$ e $b$ existem constantes $C_{1}, C_{2}, k_{1}, k_{2}$ (veja proposição $3.2 \mathrm{em}$ [Sar09]) tais que

$$
\left.C_{1} Z_{n-k_{1}}(f, a) \leq Z_{n}(f, b) \leq C_{2} Z_{n+k_{2}}(f, a) \forall n \text { (veja definição }(11)\right) .
$$

Assim como no caso compacto temos um princípio variacional para a pressão:

Teorema 2. Sejam $\left(\Sigma_{A}(\mathbb{N}), \sigma\right)$ um shift topologicamente mixing e g uma função Walters tais que $\sup g<\infty$, então

$$
P(g)=\sup \left\{h(\nu)+\int g d \nu \mid \nu \in \mathcal{M}_{\sigma}\left(\Sigma_{A}(\mathbb{N}), \int g d \nu>-\infty\right\} .\right.
$$

Onde $h(\nu)$ é a entropia de Kolmogorov-Sinai de $\nu$.

Este teorema foi provado inicialmente por O. Sarig (veja [Sar99]) para funções com variação somável sobre um shift de Markov topologicamente mixing, a prova para o caso de funções Walters é exatamente a mesma, os detalhes para o caso Walters foram escritos por Y. Daon em [Dao12].

Teorema 3 (O. Sarig, [Sar99]). Sejam $\Sigma_{A}(\mathbb{N})$ um shift topologicamente mixing e $f$ Walters, então

$$
P(\phi)=\sup \left\{P\left(\left.\phi\right|_{Y}\right): Y \text { é um shift compacto topologicamente mixing sub-sistema de } \Sigma_{A}(\mathbb{N})\right\} \text {. }
$$

Observação 1. Note que como corolário do Teorema 3 , temos que $P(\beta f) \leq \beta P(f), \forall \beta \geq 1$, isso é consequência direta do caso compacto, veja [Wal82].

Definição 8. Dado o shift $\left(\Sigma_{A}(\mathbb{N}), \sigma\right)$ e uma função $f: \Sigma_{A}(\mathbb{N}) \rightarrow \mathbb{R}$, a medida $\mu \in \mathcal{M}_{\sigma}\left(\Sigma_{A}(\mathbb{N})\right)$ é chamada de estado de equilíbrio se

$$
h(\mu)+\int_{\Sigma_{A}(\mathbb{N})} f d \mu=\sup \left\{h(\nu)+\int_{\Sigma_{A}(\mathbb{N})} f d \nu \mid \nu \in \mathcal{M}_{\sigma}\left(\Sigma_{A}(\mathbb{N})\right), \int_{\Sigma_{A}(\mathbb{N})} f d \nu>-\infty\right\} .
$$

Sobre um shift $\left(\Sigma_{A}, \sigma\right)$ com um número finito de símbolos e topologicamente mixing existe uma única medida de equilíbrio associada a cada função Hölder contínua $f: \Sigma_{A} \rightarrow \mathbb{R}$. Além disso, cada uma destas medidas de equilíbrio são também estados de Gibbs para $f$ ([Bow08, Rue78]).

Definição 9. Dizemos que uma medida de probabilidade invariante $\mu$ é uma medida de Gibbs (no sentido de Bowen) para a função $g: \Sigma_{A}(\mathbb{N}) \rightarrow \mathbb{R}$ se existem constantes $C_{1}, C_{2}>0$ tal que

$$
C_{1} \leq \frac{\mu\left[x_{0} \ldots x_{n-1}\right]}{\exp \left(S_{n} g(x)-n P(g)\right)} \leq C_{2}
$$

para cada $x \in\left[x_{0} \ldots x_{n-1}\right]$ e $n>0$.

Sobre um alfabeto infinito, medidas de Gibbs e estados de equilíbrio poderiam não existir. É sabido que para escolhas particulares de $\Sigma_{A}$ e $f$ existem (únicos) estados de equilíbrio $\mu_{\beta f}$ associados à função $\beta f$.

Teorema 4 ([BS03, Dao12]). Seja $\left(\Sigma_{A}(\mathbb{N}), \sigma\right)$ um shift topologicamente transitivo e suponha $f$ : $\Sigma_{A}(\mathbb{N}) \rightarrow \mathbb{R}$ com $\sup f<\infty, P(f)<\infty$ e Walters. Então existe no máximo uma medida de probabilidade invariante $\mu$ que é de equilíbrio. Além disso, a medida de equilíbrio quando existe pode ser obtida através do operador de Ruelle.

Definição 10. (Operador de Ruelle) Seja $f: \Sigma_{A}(\mathbb{N}) \rightarrow \mathbb{R}$ uma função. Definimos o Operador de Ruelle-Perron-Frobenius $L_{f}: C_{b}\left(\Sigma_{A}(\mathbb{N})\right) \rightarrow C_{b}\left(\Sigma_{A}(\mathbb{N})\right)$ por $\left(L_{f} g\right)(x)=\sum_{i \in \mathbb{N}} e^{f(i x)} g(i x)$. Onde $C_{b}\left(\Sigma_{A}(\mathbb{N})\right)$ é o espaço das funçôes contínuas e limitadas de valor real em $\Sigma_{A}(\mathbb{N})$. 
É claro que devemos impor restrições sobre a $f$ para que o operador esteja bem definido, uma condição usual é pedir que $\left\|L_{f} 1\right\|_{\infty}<\infty$. De fato essa hipótese nos fornece a finitude da pressão:

Teorema 5 (O. Sarig, [Sar09]). Seja $\Sigma_{A}(\mathbb{N})$ topologicamente mixing, e suponha $f: \Sigma_{A}(\mathbb{N}) \rightarrow \mathbb{R}$ Walters entâo se $\left\|L_{f} 1\right\|_{\infty}<\infty$ temos que $P(f)<\infty$.

Daremos agora uma caracterização para a existência de medidas de equilíbrio via uma generalização do Teorema de Ruelle-Perron-Frobenius.

Definição 11. Fixe $a \in \mathbb{N}$ permitido. Seja $f: \Sigma_{A}(\mathbb{N}) \rightarrow \mathbb{R}$ tal que $P(f)<\infty$.

Defina $f_{a}(x):=1_{[a]}(x) \inf \left\{n \geq 1: \sigma^{n}(x) \in[a]\right\}($ aqui $\inf \emptyset:=\infty$ e $0 \cdot \infty=0)$,

$Z_{n}(f, a):=\sum_{\substack{\sigma^{n} x=x \\ x_{0}=a}} e^{S_{n} f(x)}$ e $\quad Z_{n}^{*}(f, a):=\sum_{\sigma^{n} x=x} e^{S_{n} f(x)} 1_{\left[f_{a}=n\right]}(x)$.

Seja $\lambda=\exp P(f)$.

- $f$ é recorrente se $\sum_{n \geq 1} \lambda^{-n} Z_{n}(f, a)=\infty$.

- $f$ é positivamente recorrente se é recorrente e $\sum_{n \geq 1} n \lambda^{-n} Z_{n}^{*}(f, a)<\infty$;

- $f$ é recorrentemente nula se é recorrente e $\sum_{n \geq 1} n \lambda^{-n} Z_{n}^{*}(f, a)=\infty$.

Sempre assumiremos que $\Sigma_{A}(\mathbb{N})$ é topologicamente mixing, neste caso, a definição independe do símbolo $a$.

Teorema 6 (O. Sarig, [Sar99]). Seja $\left(\Sigma_{A}(\mathbb{N}), \sigma\right)$ um shift topologicamente mixing e $f: \Sigma_{A}(\mathbb{N}) \rightarrow \mathbb{R}$ Walters tais que $P(f)<\infty$. Então $f$ é positivamente recorrente se, e somente se, existem $\lambda>0$, uma função contínua e positiva $h$, e uma medida $\nu$ finita e positiva sobre os cilindros, tais que $L_{f} h=\lambda h$, $L_{f}^{*} \nu=\lambda \nu$ e $\int h d \nu=1$. Neste caso $\lambda=e^{P(f)}$ e $m:=h d \nu$ é uma medida de probabilidade invariante. Ademais, $m$ é a medida de equilíbrio para $f$. Além disso, para cada função uniformemente contínua $g$ tal que $\left\|g h^{-1}\right\|_{\infty}<\infty, \lambda^{-n}\left(L_{f}^{n} g\right)(x) \rightarrow h(x) \int g d \nu$ uniformemente sobre compactos.

Aqui o formalismo se distancia do caso compacto, note que a automedida a principio é finita somente em cilindros, podendo dar massa infinita ao espaço todo. De fato isso ocorrerá quando o shift não for finitamente primitivo. E ainda, sequer definimos o operador dual. A partir de agora, a menos que se diga o contrário, nos concentraremos no caso BIP. Quando $\Sigma_{A}(\mathbb{N})$ é topologicamente mixing e tem a propriedade BIP, qualquer potencial de variação somável e pressão finita é positivamente recorrente. Neste caso o operador dual é definido de maneira usual:

$$
\begin{gathered}
L_{f}^{*}: C_{b}^{*}\left(\Sigma_{A}(\mathbb{N})\right) \rightarrow C_{b}^{*}\left(\Sigma_{A}(\mathbb{N})\right) \\
\mu \rightarrow L_{f}^{*}(\mu) \\
L_{f}^{*}(\mu)(g):=\mu\left(L_{f}(g)\right)=\int L_{f}(g) d \mu \forall g \in C_{b}\left(\Sigma_{A}(\mathbb{N})\right) .
\end{gathered}
$$

Além da propriedade BIP em $\Sigma_{A}(\mathbb{N})$, assumiremos na maioria das vezes que o potencial $f: \Sigma_{A}(\mathbb{N}) \rightarrow \mathbb{R}$ tem variação somável e $P(f)<\infty$. Neste contexto temos o seguinte lema:

Lema 4. Seja $\left(\Sigma_{A}(\mathbb{N}), \sigma\right)$ um shift finitamente primitivo.

Seja $f: \Sigma_{A}(\mathbb{N}) \rightarrow \mathbb{R}$ com $\operatorname{Var}(f)<\infty$ e $P(f)<\infty$, então $\sum_{i \in \mathbb{N}} \exp \left(\left.\sup f\right|_{[i]}\right)<\infty$. 
Demonstração. Para cada $a \in \mathbb{N}$ e $n \geq K_{0}$ existem $w_{1}, w_{2}, \ldots, w_{n} \in \mathbb{F}$ tais que a sequência periódica $\overline{a w_{1} \cdots w_{n}}$ é permitida. Para cada $w_{1}, \ldots, w_{n} \in \mathbb{F}$ fixos, denotamos por $C\left(w_{1}, \ldots, w_{n}\right)$ o conjunto de símbolos $a \in \mathbb{N}$ tal que $\overline{a w_{1} \cdots w_{n}}$ é permitida. Usando que $P(f)$ é finita, temos

$$
\sum_{\sigma^{n+1}(x)=x} \exp \left(S_{n+1} f(x)\right) 1_{\left[w_{1}\right]}(x)<\infty .
$$

Isto implica que

$$
\sum_{a \in C\left(w_{1}, \ldots, w_{n}\right)} \exp \left(S_{n+1} f\left(\overline{a w_{1} \cdots w_{n}}\right)\right)<\infty
$$

Assim

$$
\sum_{a \in C\left(w_{1}, \ldots, w_{n}\right)} \exp \left[f\left(\overline{a w_{1} \cdots w_{n}}\right)+f\left(\overline{w_{1} \cdots w_{n} a}\right)+\cdots+f\left(\overline{w_{n} a w_{1} \cdots w_{n-1}}\right)\right]<\infty .
$$

Note que $w_{1}, \ldots, w_{n} \in \mathbb{F}$ (finito) e $\operatorname{Var}(f)<\infty$, então

$$
f\left(\overline{w_{1} \ldots w_{n} a}\right)+\cdots+f\left(\overline{w_{n} a w_{1} \cdots w_{n-1}}\right)
$$

é limitado por uma constante que não depende de $a, w_{1}, \ldots, w_{n}$.

Portanto da equação (2.4) segue que

$$
\sum_{a \in C\left(w_{1}, \ldots, w_{n}\right)} \exp \left(f\left(\overline{a w_{1} \cdots w_{n}}\right)\right)<\infty
$$

e multiplicando por $e^{\operatorname{Var}_{1}(f)}$ obtemos

$$
\sum_{a \in C\left(w_{1}, \ldots, w_{n}\right)} \exp \left(\left.\sup f\right|_{[a]}\right)<\infty
$$

Cada $a \in \mathbb{N}$ pertence ao pelo menos um dos conjuntos $C\left(w_{1}, \ldots, w_{n}\right)$, portanto somando sobre o conjunto finito palavras $w_{1} \ldots w_{n} \in \mathbb{F}^{n}$ resulta

$$
\sum_{i \in \mathbb{N}} \exp \left(\left.\sup f\right|_{[i]}\right)<\infty
$$

Note que no caso BIP e topologicamente mixing vale a reciproca do lema acima (veja o Teorema 5). Portanto vale a equivalência, ou seja:

$$
P(f)<\infty \Leftrightarrow \sum_{i \in \mathbb{N}} \exp \left(\left.\sup f\right|_{[i]}\right)<\infty .
$$

Observe que coercividade segue da condição (2.5). Mais precisamente:

Definição 12. Um potencial continuo $f: \Sigma_{A}(\mathbb{N}) \rightarrow \mathbb{R}$ é chamado coercivo quando

$$
\left.\lim _{i \rightarrow+\infty} \sup f\right|_{[i]}=-\infty .
$$

Além disso, para potenciais Markov (que dependem de um número finito de coordenadas) com variação somável e pressão finita, a condição (2.5) implica a existência de medidas de equilíbrio em shifts topologicamente mixing (veja [VJ15]).

Com as mesmas hipóteses do lema anterior temos o seguinte teorema:

Teorema 7 (R.D. Mauldin e M. Urbański, [MU03, MU01]). Seja $\left(\Sigma_{A}(\mathbb{N}), \sigma\right)$ um shift finitamente primitivo, $f: \Sigma_{A}(\mathbb{N}) \rightarrow \mathbb{R}$ com variação somável e $P(f)<\infty$. Então $f$ tem uma única medida de 
equilíbrio $\mu_{f}$. Esta medida é Gibbs e satisfaz

$$
e^{-4 \operatorname{Var}(f)} \leq \frac{\mu_{f}\left(\left[x_{0} \ldots x_{n-1}\right]\right)}{e^{S_{n} f(x)-n P(f)}} \leq e^{4 \operatorname{Var}(f)}
$$

para cada $x \in\left[x_{0} \ldots x_{n-1}\right]$ e $n>0$.

Talvez seja o momento de uma breve discussão sobre a hipótese do shift ser finitamente primitivo. Esta pode parecer restritiva, no entanto, se nos fixamos na classe de potenciais de variação somável como pressão finita e shifts topologicamente mixing a existência de uma medida de Gibbs é equivalente a propriedade BIP. Ou seja vale o seguinte teorema provado por Sarig:

Teorema 8 (O. Sarig, [Sar03]). Seja $\left(\Sigma_{A}(\mathbb{N}), \sigma\right)$ topologicamente mixing e $f$ com variação somável, então $f$ tem uma medida de Gibbs invariante se e somente se $A$ tem a propriedade BIP e $P(f)<\infty$.

Corolário 1. Seja $\left(\Sigma_{A}(\mathbb{N}), \sigma\right)$ topologicamente mixing, $f$ com variação somável e $P(f)<\infty$. Suponha que $\Sigma_{A}$ não tenha a propriedade BIP. Então se $\mu$ é uma medida de equilíbrio para $f, \mu$ não é Gibbs.

Shifts Renewal com potencial localmente Hölder contínuos satisfazem as hipóteses do corolário acima.

A teoria das medidas de Gibbs recebe diferentes enfoques das comunidades de Teoria Ergódica e Mecânica Estatística, enquanto na Teoria Ergódica é comum encontrarmos a definição de medida de Gibbs no sentido de Bowen, na Mecânica Estatística é usual serem usadas equações de DLR e probabilidades condicionais para se definir o que é uma medida de Gibbs. Neste sentido, o Formalismo Termodinâmico para shifts não compactos é descrito de uma maneira unificada por Sarig em [Sar09]. Recentemente, A. Lopes e L. Cioletti [LC14] mostraram a equivalência das várias diferentes definições usadas pelos autores das duas comunidades no contexto de shifts compactos e potenciais Walters.

Potenciais de variação somável e pressão finita definidos em shifts topologicamente mixing com a propriedade BIP possuem medidas de equilíbrio que são Gibbs, tais medidas são da forma $h d \nu$ onde $\nu$ é uma medida de probabilidade e $h$ é a autofunção do operador de Ruelle associada ao autovalor $\lambda=e^{P(f)}, h$ é positiva e afastada uniformemente de zero e do infinito. Denotamos por $h_{\beta}$ a autofunção associada ao potencial $\beta f$.

Agora vamos explicitar cotas superiores e inferiores para a autofunção $h_{\beta}$, associada ao potencial $\beta f$. Estas cotas serão usadas posteriormente quando fizermos a temperatura ir para zero.

Proposição 1. Seja $\left(\Sigma_{A}(\mathbb{N}), \sigma\right)$ um shift finitamente primitivo, $f: \Sigma_{A}(\mathbb{N}) \rightarrow \mathbb{R}$ com variação somável tal que $P(f)<\infty$. Então para qualquer $x, y \in \Sigma_{A}(\mathbb{N})$ tal que $d(x, y)<1$ temos

$$
h_{\beta}(x) \leq h_{\beta}(y) e^{\beta \operatorname{Var}(f)},
$$

onde $h_{\beta}$ é a autofunção do operador de Ruelle associada a $\beta f$.

Demonstração. Provar que $h_{\beta}(x) \leq h_{\beta}(y) e^{\beta \operatorname{Var}(f)}$ é equivalente a provar que

$$
h_{\beta}(x)-h_{\beta}(y) e^{\beta \operatorname{Var}(f)} \leq 0 .
$$

Sabemos que $\lim _{n \rightarrow \infty} \frac{1}{\lambda_{\beta}^{n}} L_{\beta f}^{n} 1(x)=h_{\beta}(x)$ uniformemente em compactos (veja [Sar99]), onde $\lambda_{\beta}$ é o autovalor do operador de Ruelle associado à autofunção $h_{\beta}$.

Para cada $x \in \Sigma_{A}(\mathbb{N})$ definimos

$$
P^{n}(x)=\left\{p=\left(p_{0}, \ldots, p_{n-1}\right): p x \in \Sigma_{A}(\mathbb{N})\right\} .
$$


Por hipótese $d(x, y)<1$ assim $P^{n}(x)=P^{n}(y)$. Então

$$
\begin{aligned}
& h_{\beta}(x)-h_{\beta}(y) e^{\beta \operatorname{Var}(f)}=\lim _{n \rightarrow \infty} \frac{1}{\lambda_{\beta}^{n}} L_{\beta f}^{n} 1(x)-\lim _{n \rightarrow \infty} \frac{1}{\lambda_{\beta}^{n}} L_{\beta f}^{n} 1(y) e^{\beta \operatorname{Var}(f)} \\
& =\lim _{n \rightarrow \infty} \frac{1}{\lambda_{\beta}^{n}}\left(L_{\beta f}^{n} 1(x)-L_{\beta f}^{n} 1(y) e^{\beta \operatorname{Var}(f)}\right) \\
& =\lim _{n \rightarrow \infty} \frac{1}{\lambda_{\beta}^{n}}\left(\sum_{p \in P^{n}(x)} e^{\beta S_{n} f(p x)}-e^{\beta S_{n} f(p y)+\beta \operatorname{Var}(f)}\right) \\
& =\lim _{n \rightarrow \infty} \frac{1}{\lambda_{\beta}^{n}} \sum_{p \in P^{n}(x)} e^{\beta S_{n} f(p y)}\left[e^{\beta S_{n} f(p x)-\beta S_{n} f(p y)}-e^{\beta \operatorname{Var}(f)}\right]
\end{aligned}
$$

Note que

$$
e^{\beta S_{n} f(p x)-\beta S_{n} f(p y)}-e^{\beta \operatorname{Var}(f)} \leq 0,
$$

pois

$$
\begin{aligned}
S_{n} f(p x)-S_{n} f(p y) & =(f(p x)-f(p y))+\cdots+\left(f\left(p_{n-1} x\right)-f\left(p_{n-1} y\right)\right) \\
& \leq \operatorname{Var}_{n+t(x, y)}(f)+\cdots+\operatorname{Var}_{1+t(x, y)}(f) \\
& \leq \operatorname{Var}(f) .
\end{aligned}
$$

Assim concluímos que $h_{\beta}(x) \leq h_{\beta}(y) e^{\beta \operatorname{Var}(f)}$.

Usando um argumento similar à prova da proposição acima temos que, o resultado da proposição anterior também é verdadeiro para potenciais Walters com $\operatorname{Var}_{1}(f)<\infty$, a saber:

Proposição 2. Seja $\left(\Sigma_{A}(\mathbb{N}), \sigma\right)$ um shift finitamente primitivo, $f: \Sigma_{A}(\mathbb{N}) \rightarrow \mathbb{R}$ Walters com $\operatorname{Var}_{1}(f)<\infty$ tal que $P(f)<\infty$. Então para qualquer $x, y \in \Sigma_{A}(\mathbb{N})$ tal que $d(x, y)<1$ temos

$$
h_{\beta}(x) \leq h_{\beta}(y) e^{\beta M_{0}},
$$

onde $M_{0}:=\sup _{n \geq 1} \operatorname{Var}_{n+1} S_{n} f$ e $h_{\beta}$ é a autofunção do operador de Ruelle associada a $\beta f$.

A seguinte proposição mostra que $h_{\beta}$ está afastada uniformemente de zero e do infinito:

Proposição 3. Seja $\left(\Sigma_{A}(\mathbb{N}), \sigma\right)$ finitamente primitivo e $f: \Sigma_{A}(\mathbb{N}) \rightarrow \mathbb{R}$ com variação somável e $P(f)<\infty$. Então existem constantes $C_{1}, C_{2} \in \mathbb{R}$ tais que

$$
e^{C_{1} \beta} \leq h_{\beta} \leq e^{C_{2} \beta}, \quad \forall \beta \geq 1 .
$$

Demonstração. Fixe $\epsilon>0$. Seja $x \in \Sigma_{A}(\mathbb{N})$, como $\int h_{\beta} d \nu_{\beta}=1$, e $\nu_{\beta}$ é uma medida de probabilidade existe $y=\left(y_{0}, y_{1}, \ldots\right) \in \Sigma_{A}(\mathbb{N})$ tal que $h_{\beta}(y)>1-\epsilon$. Usando que $\Sigma_{A}(\mathbb{N})$ é finitamente primitivo sabemos que existe uma palavra $w:=y_{0} w_{0} \ldots w_{K_{0}-1}$, com $w_{0}, \ldots, w_{K_{0}-1} \in \mathbb{F}$. Definimos $z:=w x$, assim $d(y, z)<1$, portanto pela Proposição 1 temos que

$$
\frac{h_{\beta}(y)}{h_{\beta}(z)} \leq e^{\beta \operatorname{Var}(f)}
$$

Assim usando (2.10) e $P(\beta f) \leq \beta P(f), \forall \beta \geq 1$ (veja teorema 3 e [Wal82]) segue que

$$
\begin{aligned}
h_{\beta}(x)=\frac{L_{\beta f}^{K_{0}+1} h_{\beta}(x)}{\lambda_{\beta}^{K_{0}+1}} & \geq e^{\beta S_{K_{0}+1}(f)(z)-\left(K_{0}+1\right) P(\beta f)} h_{\beta}(z) \\
& \geq e^{\beta S_{K_{0}+1}(f)(z)-\left(K_{0}+1\right) \beta P(f)} h_{\beta}(y) e^{-\beta \operatorname{Var}(f)} .
\end{aligned}
$$


Observe que como $f$ tem variação somável podemos encontrar um $x^{\prime}$ pertencendo ao compacto $\Sigma_{A}(\mathbb{F})$, tais que

$$
\begin{aligned}
S_{K_{0}+1}(f)(z) & =f\left(y_{0} w_{0} \ldots w_{K_{0}-1} x\right)+f\left(w_{0} \ldots w_{K_{0}-1} x\right)+\cdots+f\left(w_{K_{0}-1} x\right) \\
& >f\left(y_{0} w_{0} \ldots w_{K_{0}-1} x^{\prime}\right)+\cdots+f\left(w_{K_{0}-1} x^{\prime}\right)-\operatorname{Var}(f) \\
& >\left(K_{0}+1\right) m-\operatorname{Var}(f),
\end{aligned}
$$

onde

$$
m:=\min \left\{f\left(y_{0} w_{0} \ldots w_{K_{0}-1} x^{\prime}\right), f\left(w_{0} \ldots w_{K_{0}-1} x^{\prime}\right), f\left(w_{K_{0}-1} x^{\prime}\right) ; 1 \leq w_{j} \leq N\right\},
$$

aqui $N:=I_{\mathbb{F}}$ (veja equação $(2.3)$ ). Deste modo obtemos que

$$
h_{\beta}(x) \geq e^{\beta\left(\left(K_{0}+1\right) m-2 \operatorname{Var}(f)-\left(K_{0}+1\right) P(f)\right)} h_{\beta}(y) .
$$

Usando que $h_{\beta}(y)>1-\epsilon$ concluímos que

$$
(1-\epsilon) e^{\beta C_{1}} \leq h_{\beta}(x)
$$

onde $C_{1}:=\left(K_{0}+1\right) m-2 \operatorname{Var}(f)-\left(K_{0}+1\right) P(f)$. Fazendo $\epsilon \rightarrow 0$ concluímos que $e^{C_{1} \beta} \leq h_{\beta}(x), \forall x$.

Agora provaremos a outra desigualdade. Fixemos $a \in \mathbb{N}$. Provaremos primeiro que $h_{\beta}(x)$ está afastada de infinito para todo $x \in[a]$. Com efeito: como $\int h_{\beta} d \nu_{\beta}=1$, existe $y \in \Sigma_{A}(\mathbb{N})$ tal que $h_{\beta}(y)<1+\epsilon$. Usando a hipóteses de finitamente primitivo sabemos que existe uma palavra $w:=a w_{0} \ldots w_{K_{0}-1}, \operatorname{com} w_{0}, \ldots, w_{K_{0}-1} \in \mathbb{F}$. Seja $z^{\prime}:=w y$, assim $d\left(z^{\prime}, x\right)<1$ portanto pela Proposição 1 temos que

$$
\frac{h_{\beta}(x)}{h_{\beta}\left(z^{\prime}\right)} \leq e^{\beta \operatorname{Var}(f)} .
$$

Logo usando a equação (2.12) e $P(\beta f) \leq \beta P(f), \forall \beta \geq 1$ (veja teorema 3 e [Wal82]) segue que

$$
\begin{aligned}
h_{\beta}(y)=\frac{L_{\beta f}^{K_{0}+1} h_{\beta}(y)}{\lambda_{\beta}^{K_{0}+1}} & \geq e^{\beta S_{K_{0}+1}(f)\left(z^{\prime}\right)-\left(K_{0}+1\right) P(\beta f)} h_{\beta}\left(z^{\prime}\right) \\
& \geq e^{\beta S_{K_{0}+1}(f)\left(z^{\prime}\right)-\left(K_{0}+1\right) \beta P(f)} h_{\beta}(x) e^{-\beta \operatorname{Var}(f)} .
\end{aligned}
$$

Definimos $f_{n}\left(d_{0} \ldots d_{n-1}\right):=\inf \left\{S_{n} f(x): x \in\left[d_{0} \ldots d_{n-1}\right]\right\}$. Afirmação: $f_{n}\left(d_{0} \ldots d_{n-1}\right)>-\infty$. Com efeito

$$
\begin{aligned}
f_{n}\left(d_{0} \ldots d_{n-1}\right) & \geq \inf _{x \in\left[d_{0} \ldots d_{n-1}\right]} f(x)+\cdots+\inf _{x \in\left[d_{n-1}\right]} f(x) \\
& \geq n \inf _{x \in\left[d_{n-1}\right]} f(x) \\
& \geq n\left(f\left(d_{n-1} \ldots\right)-\operatorname{Var}_{1}(f)\right)>-\infty
\end{aligned}
$$

Seja $\bar{C}:=\min \left\{\inf _{x \in\left[a w_{0} \ldots w_{k_{0}-1}\right]} S_{K_{0}+1} f(x): 1 \leq w_{j} \leq N\right\}$. Note que $S_{K_{0}+1}(f)\left(z^{\prime}\right) \geq \bar{C}$. Deste modo obtemos que

$$
h_{\beta}(x) \leq(1+\epsilon) e^{\beta \bar{C}_{2}},
$$

onde $\bar{C}_{2}:=-\bar{C}+\left(K_{0}+1\right) P(f)+\operatorname{Var}(f)$, observe que $\bar{C}_{2}$ depende de $a$. Fazendo $\epsilon \rightarrow 0$ segue que

$$
h_{\beta}(x) \leq e^{\beta \bar{C}_{2}} \text {, para cada } x \in[a] .
$$

Agora vejamos que $h_{\beta}$ está longe de infinito para todo $x \in \Sigma_{A}(\mathbb{N})$. 
Fixar algum $z^{i} \in\left[w_{i}\right]\left(0 \leq i \leq K_{0}-1\right)$. Para cada ponto da forma $\left(\xi_{0}, \ldots, \xi_{n-1}, x\right)$ podemos encontrar $0 \leq i \leq K_{0}-1$ tais que $\left(\xi_{0}, \ldots, \xi_{n-1}, z^{i}\right)$ é uma sequência permitida. Já que $f$ tem variação somável temos que

$$
S_{n} f\left(\xi_{0}, \ldots, \xi_{n-1}, x\right)-S_{n} f\left(\xi_{0}, \ldots, \xi_{n-1}, z^{i}\right) \leq \operatorname{Var}(f) .
$$

Logo

$$
S_{n} f\left(\xi_{0}, \ldots, \xi_{n-1}, x\right) \leq \operatorname{Var}(f)+S_{n} f\left(\xi_{0}, \ldots, \xi_{n-1}, z^{i}\right),
$$

assim

$$
e^{\beta S_{n} f\left(\xi_{0}, \ldots, \xi_{n-1}, x\right)} \leq e^{\beta \operatorname{Var}(f)} e^{\beta S_{n} f\left(\xi_{0}, \ldots, \xi_{n-1}, z^{i}\right)},
$$

deste modo

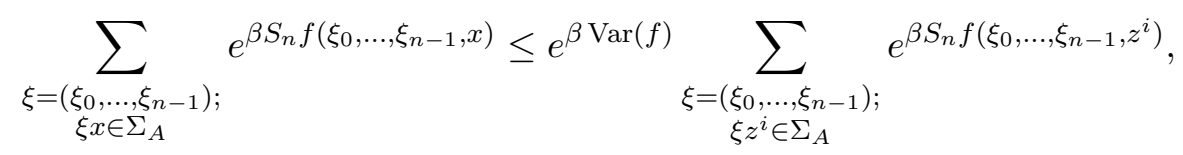

assim temos que

$$
\lambda_{\beta}^{-n}\left(L_{\beta f}^{n} 1\right)(x) \leq e^{\beta \operatorname{Var}(f)} \lambda_{\beta}^{-n}\left(L_{\beta f}^{n} 1\right)\left(z^{i}\right) .
$$

Logo tomando o limite quando $n \rightarrow \infty$ obtemos

$$
h_{\beta}(x) \leq e^{\beta \operatorname{Var}(f)} h_{\beta}\left(z^{i}\right) .
$$

Agora usando que $h_{\beta}\left(z^{i}\right) \leq e^{\beta \bar{C}_{2}}$, para cada $z^{i} \in\left[w_{i}\right]$ com $\bar{C}_{2}=\bar{C}_{2}\left(z^{i}\right)$. Concluímos que

$$
h_{\beta}(x) \leq e^{\beta C_{2}} .
$$

Onde $C_{2}:=\max \left\{\bar{C}_{2}\left(z^{i}\right) ; 0 \leq i \leq K_{0}-1\right\}+\operatorname{Var}(f)$.

Além disso, neste caso onde $f$ tem variação somável e $P(f)<\infty$ com $\Sigma_{A}(\mathbb{N})$ finitamente primitivo, sempre podemos normalizar o operador para cada $\beta>0$, ou seja:

Definição 13. Dizemos que o operador de Ruelle está normalizado quando existe uma função $g$ tais que $L_{g} 1=1$. Observe que a função $g_{\beta}:=\beta f+\log h_{\beta}-\log \left(h_{\beta} \circ \sigma\right)-P(\beta f)$ satisfaz $L_{g_{\beta}} 1=1$. Com efeito

$$
\begin{aligned}
L_{g_{\beta}} 1 & =\sum_{i=0}^{\infty} e^{\beta f(i x)} e^{\log h_{\beta}(i x)} e^{-\log h_{\beta}(\sigma(i x))} e^{-P(\beta f)} \\
& =\frac{1}{h_{\beta}(x)} \sum_{i=0}^{\infty} e^{\beta f(i x)} \frac{h_{\beta}(i x)}{\lambda_{\beta}} \\
& =\frac{L_{\beta f} h_{\beta}}{\lambda_{\beta} h_{\beta}} \\
& =1 .
\end{aligned}
$$

Lema 5. Sob as hipóteses da Proposição 1 a função $\varphi_{\beta}:=\frac{1}{\beta} \log h_{\beta}$ tem variação somável.

Demonstração. Sejam $x, y \in \Sigma_{A}(\mathbb{N}) \operatorname{com} d(x, y) \leq r^{m}, m \geq 1$, usando um argumento similar à prova da Proposição 1 (veja equação (2.8)) obtemos que, para todo $n \geq 1$,

$$
h_{\beta}(x) \leq h_{\beta}(y) e^{\beta\left[\operatorname{Var}_{n+m}(f)+\cdots+\operatorname{Var}_{m+1}(f)\right]},
$$

assim

$$
\varphi_{\beta}(x)-\varphi_{\beta}(y) \leq \operatorname{Var}_{n+m}(f)+\cdots+\operatorname{Var}_{m+1}(f)
$$


deste modo

$$
\operatorname{Var}_{m}\left(\varphi_{\beta}\right) \leq \operatorname{Var}_{n+m}(f)+\cdots+\operatorname{Var}_{m+1}(f)
$$

Logo

$$
\operatorname{Var}\left(\varphi_{\beta}\right)=\sum_{m=1}^{\infty} \operatorname{Var}_{m}\left(\varphi_{\beta}\right) \leq \sum_{m=1}^{\infty}\left(\operatorname{Var}_{n+m}(f)+\cdots+\operatorname{Var}_{m+1}(f)\right)
$$

e já que $f$ tem variação somável temos que

$$
\sum_{m=1}^{\infty} \operatorname{Var}_{m+n}(f)<\infty, \ldots, \sum_{m=1}^{\infty} \operatorname{Var}_{m+1}(f)<\infty,
$$

portanto

$$
\operatorname{Var}\left(\varphi_{\beta}\right)<\infty
$$

Lema 6. Sob as hipóteses da Proposição 2 a função $\varphi_{\beta}:=\frac{1}{\beta} \log h_{\beta}$ tem a propriedade Walters.

Demonstração. Sejam $x, y \in \Sigma_{A}(\mathbb{N}) \operatorname{com} d(x, y) \leq r^{m+k}, m, k \geq 1$ afirmamos que

$$
h_{\beta}(x) \leq h_{\beta}(y) e^{\beta \sup _{l \geq 1} \operatorname{Var}_{m+k+l} S_{l} f} .
$$

Observe que, provar (2.16) é equivalente a provar que, para todo $n \geq 1$, a seguinte desigualdade: (veja Proposição 1)

$$
e^{\beta S_{n} f(p x)-\beta S_{n} f(p y)}-e^{\beta \sup _{l \geq 1} \operatorname{Var}_{m+k+l} S_{l} f} \leq 0 .
$$

e esta última desigualdade é verdadeira pois

$$
\begin{aligned}
S_{n} f(p x)-S_{n} f(p y) & =\left[f\left(p_{0} \ldots p_{n-1} x\right)-f\left(p_{0} \ldots p_{n-1} y\right)\right]+\cdots+\left[f\left(p_{n-1} x\right)-f\left(p_{n-1} y\right)\right] \\
& \leq \sup _{l \geq 1} \operatorname{Var}_{m+k+l} S_{l} f .
\end{aligned}
$$

Similarmente afirmamos que se $d(x, y) \leq r^{m+k}, m, k \geq 1$ então

$$
h_{\beta}\left(\sigma^{m-1}(x)\right) \leq h_{\beta}\left(\sigma^{m-1}(y)\right) e^{\beta \sup _{l \geq 1} \operatorname{Var}_{k+1+l} S_{l} f} .
$$

Observe que provar (2.17) é equivalente a provar a seguinte desigualdade: (veja Proposição 1)

$$
e^{\beta S_{n} f\left(p \sigma^{m-1}(x)\right)-\beta S_{n} f\left(p \sigma^{m-1}(y)\right)}-e^{\beta \sup _{l \geq 1} \operatorname{Var}_{k+1+l} S_{l} f} \leq 0 .
$$

e esta última desigualdade é verdadeira pois

$$
\begin{aligned}
& S_{n} f\left(p \sigma^{m-1}(x)\right)-S_{n} f\left(p \sigma^{m-1}(y)\right) \\
& =\left[f\left(p_{0} \ldots p_{n-1} \sigma^{m-1}(x)\right)-f\left(p_{0} \ldots p_{n-1} \sigma^{m-1}(y)\right)\right]+\cdots+\left[f\left(p_{n-1} \sigma^{m-1}(x)\right)-f\left(p_{n-1} \sigma^{m-1}(y)\right)\right] \\
& \leq \sup _{l \geq 1} \operatorname{Var}_{k+1+l} S_{l} f .
\end{aligned}
$$

Desta maneira usando (2.16) e (2.17) segue que

$$
\begin{aligned}
& S_{n} \varphi_{\beta}(x)-S_{n} \varphi_{\beta}(y) \\
& =\left[\frac{1}{\beta} \log h_{\beta}(x)-\frac{1}{\beta} \log h_{\beta}(y)\right]+\cdots+\left[\frac{1}{\beta} \log h_{\beta}\left(\sigma^{n-1}(x)\right)-\frac{1}{\beta} \log h_{\beta}\left(\sigma^{n-1}(y)\right)\right] \\
& \leq \sup _{l \geq 1} \operatorname{Var}_{k+1+l} S_{l} f .
\end{aligned}
$$


Portanto

$$
\operatorname{Var}_{n+k} S_{n} \varphi_{\beta} \leq \sup _{l \geq 1} \operatorname{Var}_{k+1+l} S_{l} f
$$

donde

$$
\sup _{n \geq 1} \operatorname{Var}_{n+k} S_{n} \varphi_{\beta} \leq \sup _{l \geq 1} \operatorname{Var}_{k+1+l} S_{l} f<\infty
$$

Ademais

$$
\lim _{k \rightarrow \infty} \sup _{n \geq 1} \operatorname{Var}_{n+k} S_{n} \varphi_{\beta} \leq \lim _{k \rightarrow \infty} \sup _{l \geq 1} \operatorname{Var}_{k+1+l} S_{l} f=0 \text { ( } f \text { é Walters). }
$$

Portanto concluímos que $\varphi_{\beta}$ tem a propriedade Walters.

Observação 2. Note que $\operatorname{Var}_{1}\left(\frac{1}{\beta} \log h_{\beta}\right)<\infty$. Com efeito Se $x_{0}=y_{0}$ segue que (veja equação $(2.8)$ )

$$
\frac{1}{\beta} \log h_{\beta}(x)-\frac{1}{\beta} \log h_{\beta}(y) \leq M_{0}<\infty .
$$

Lema 7. Sob as hipóteses da Proposição 1 a função $\phi_{\beta}:=\frac{g_{\beta}}{\beta}$ tem variação somável.

Demonstração. Sejam $x, y \in \Sigma_{A}(\mathbb{N}) \operatorname{com} d(x, y) \leq r^{m},(m \geq 1)$. Então

$$
\begin{aligned}
& \phi_{\beta}(x)-\phi_{\beta}(y) \\
& =(f(x)-f(y))+\frac{1}{\beta}\left(\log h_{\beta}(x)-\log h_{\beta}(y)\right)-\frac{1}{\beta}\left(\log h_{\beta}(\sigma(x))-\log h_{\beta}(\sigma(y))\right) \\
& \leq \operatorname{Var}_{m}(f)+\operatorname{Var}_{m}\left(\frac{1}{\beta} \log h_{\beta}\right)+\operatorname{Var}_{m}\left(\frac{1}{\beta} \log h_{\beta} \circ \sigma\right) .
\end{aligned}
$$

Assim

$$
\operatorname{Var}_{m}\left(\phi_{\beta}\right) \leq \operatorname{Var}_{m}(f)+\operatorname{Var}_{m}\left(\frac{1}{\beta} \log h_{\beta}\right)+\operatorname{Var}_{m}\left(\frac{1}{\beta} \log h_{\beta} \circ \sigma\right),
$$

e usando que $f$ e $\frac{1}{\beta} \log h_{\beta}$ tem variação somável obtemos

$$
\sum_{m=1}^{\infty} \operatorname{Var}_{m}\left(\phi_{\beta}\right) \leq \sum_{m=1}^{\infty} \operatorname{Var}_{m}(f)+\sum_{m=1}^{\infty} \operatorname{Var}_{m}\left(\frac{1}{\beta} \log h_{\beta}\right)+\sum_{m=1}^{\infty} \operatorname{Var}_{m}\left(\frac{1}{\beta} \log h_{\beta} \circ \sigma\right)<\infty .
$$

Em consequência $\phi_{\beta}$ tem variação somável.

Observe $g_{\beta}$ tem variação somável e pressão finita, de fato $P\left(g_{\beta}\right)=0$.

Desta forma, pelo exposto acima $\beta f$ é cohomóloga (veja definição 16) a $g_{\beta}$ e portanto possuem as mesmas medidas de equilíbrio. Note que neste ponto usamos de maneira fundamental a propriedade BIP de $\Sigma_{A}(\mathbb{N})$ pois na ausência desta a autofunção $h_{\beta}$ pode se aproximar de zero. Em particular temos o seguinte teorema:

Proposição 4. Seja $\left(\Sigma_{A}(\mathbb{N}), \sigma\right)$ um shift topologicamente mixing satisfazendo a propriedade BIP. Seja $f: \Sigma_{A}(\mathbb{N}) \rightarrow \mathbb{R}$ uma função com variação somável e com $P(f)<\infty$. Suponha ademais que o operador de Ruelle está normalizado. Então para $\mu=L_{f}^{*}(\mu)$ medida de equilíbrio, verificamos

$$
\mu\left(\left[x_{1} \ldots x_{n}\right]\right)=\int_{\left[x_{0} \ldots x_{n}\right]} e^{-f} d \mu .
$$

Ademais temos a seguinte desigualdade:

$$
e^{-\operatorname{Var}_{n+1}(f)} \leq \frac{\mu\left[x_{0} \ldots x_{n}\right]}{\mu\left[x_{1} \ldots x_{n}\right]} e^{-f(x)} \leq e^{\operatorname{Var}_{n+1}(f)}, x \in\left[x_{0} \ldots x_{n}\right] .
$$


Em particular

$$
\left(\inf _{z \in\left[x_{0} \ldots x_{n}\right]} e^{-f(z)}\right) \mu\left(\left[x_{0} \ldots x_{n}\right]\right) \leq \mu\left(\left[x_{1} \ldots x_{n}\right]\right) \leq\left(\sup _{z \in\left[x_{0} \ldots x_{n}\right]} e^{-f(z)}\right) \mu\left(\left[x_{0} \ldots x_{n}\right]\right) .
$$

Demonstração. Note que

$$
f(z)-f(x) \leq \operatorname{Var}_{n+1}(f), \text { sempre que } z, x \in\left[x_{0}, \ldots, x_{n}\right],
$$

então, fixado $z$, temos que para todo $x \in\left[x_{0}, \ldots, x_{n}\right]$ :

$$
-f(x) \leq \operatorname{Var}_{n+1}(f)-f(z),
$$

portanto

$$
\sup _{x \in\left[x_{0}, \ldots, x_{n}\right]} e^{-f(x)} \leq e^{\operatorname{Var}_{n+1}(f)-f(z)}<\infty .
$$

Observe que como $\mu$ é uma medida invariante temos

$$
\mu\left(\left[x_{1} \ldots x_{n}\right]\right)=\mu\left(\sigma^{-1}\left[x_{1} \ldots x_{n}\right]\right)=\sum_{i=0}^{\infty} \mu\left[i x_{1} \ldots x_{n}\right] .
$$

Assim

$$
\begin{aligned}
\mu\left(\left[x_{1} \ldots x_{n}\right]\right)=\int 1_{\left[x_{1} \ldots x_{n}\right]}(z) d \mu & =\int \sum_{i=0}^{\infty} 1_{\left[i x_{1} \ldots x_{n}\right]}(z) d \mu \\
& =\int \sum_{\substack{y \in \sigma^{-1}(z) \\
z \in\left[x_{1} \ldots x_{n}\right]}}^{\infty} 1_{\left[x_{0} \ldots x_{n}\right]}(y) d \mu \\
& =\int \sum_{\sigma y=z} e^{f(y)} 1_{\left[x_{0} \ldots x_{n}\right]}(y) e^{-f(y)} d \mu \\
& =\int L_{f}\left(1_{\left[x_{0} \ldots x_{n}\right]} e^{-f}\right)(z) d \mu \\
& \left.=\int 1_{\left[x_{0} \ldots x_{n}\right]}(z) e^{-f(z)} d \mu \quad \text { aqui } \mu=L_{f}^{*}(\mu)\right) \\
& =\int_{\left[x_{0} \ldots x_{n}\right]} e^{-f(z)} d \mu .
\end{aligned}
$$

Note que $|f(z)-f(w)| \leq \operatorname{Var}_{n+1}(f)$, sempre que $z, x \in\left[x_{0}, \ldots, x_{n}\right]$, portanto

$$
e^{-\operatorname{Var}_{n+1}(f)} \leq e^{f(z)-f(x)} \leq e^{\operatorname{Var}_{n+1}(f)},
$$

$\operatorname{logo}$ fixando $z$ e integrando em relação a $x$ resulta

$$
\begin{gathered}
\int_{\left[x_{0} \ldots x_{n}\right]} e^{-\operatorname{Var}_{n+1}(f)} d \mu \leq \int_{\left[x_{0} \ldots x_{n}\right]} e^{f(z)-f(x)} d \mu \leq \int_{\left[x_{0} \ldots x_{n}\right]} e^{\operatorname{Var}_{n+1}(f)} d \mu \\
\mu\left(\left[x_{0} \ldots x_{n}\right]\right) e^{-\operatorname{Var}_{n+1}(f)} \leq \mu\left(\left[x_{1} \ldots x_{n}\right]\right) e^{f(z)} \leq \mu\left(\left[x_{0} \ldots x_{n}\right]\right) e^{\operatorname{Var}_{n+1}(f)} .
\end{gathered}
$$




\subsection{Temperatura Zero}

Nesta seção discutimos rapidamente resultados sobre o comportamento dos objetos de interesse quando $\beta$ vai para infinito, ou seja vamos estar interessados em descrever o que acontece com pressão, medidas de equilíbrio e autofunção quando a temperatura vai para zero.

Uma pergunta natural é saber o que acontece com as medidas $\mu_{\beta f}$ quando $\beta$ tende a infinito. Observe que no contexto não compacto se um potencial $\beta f$ localmente Hölder contínuo definido sobre um shift topologicamente mixing é positivamente recorrente, nada podemos dizer sobre $\tilde{\beta} f$ se $\tilde{\beta}>\beta$. De fato é o caso do Renewal shift, há potencias para os quais existe $\beta_{c}$ tal que para $\beta<\beta_{c}$ $\beta f$ é positivamente recorrente e portanto possui uma medida de equilíbrio, ao mesmo tempo que não existe medida de equilíbrio para qualquer $\beta$ maior que $\beta_{c}$, veja [Sar01].

O que ocorre no caso não compacto é que não somente a regularidade do potencial influencia na termodinâmica, mas também seu comportamento no infinito, como coercividade, podem estar relacionados com o fenômeno de transição de fase.

Em nosso texto focamos no caso finitamente primitivo, assumindo que o potencial tem pressão finita e variação somável onde não ocorrem transições de fase e o problema de estudar a familia de medidas de equilíbrio $\mu_{\beta f}$ quando $\beta \rightarrow \infty$ faz sentido.

A interpretação termodinâmica do parâmetro $\beta$ é que este representa o inverso da temperatura de um sistema, enquanto as medidas $\mu_{\beta f}$ descrevem os equilíbrios do sistema à temperatura $1 / \beta$. O limite quando $\beta \rightarrow \infty$ é portanto o limite à temperatura zero.

Sabemos que no caso de uma única medida maximizante, $\mu_{\beta f}$ convergirá para essa medida. No entanto, na situação quando existem várias medidas maximizantes só sabemos que $\mu_{\beta f}$ vai se acumular em algum subconjunto não vazio de tais medidas.

Os resultados para temperatura zero concentram-se em entender a convergência ou não da sequência de medidas $\left(\mu_{\beta f}\right)_{\beta}$, propriedades das medidas limite e obtenção de princípios de grandes desvios para esta família. Na questão de convergência os trabalhos de Brémont [Bré03] e Chazottes, Gambaudo e Ugalde [CGU11] mostram que tem convergência quando o potencial depende de um número finito de coordenadas. R. Leplaideur em [Lep05] mostra que $\mu_{\beta f+g}$ converge, donde $f$ é localmente constante e $g$ é Hölder contínua. Todos esses resultados no contexto de shifts com um número finito de símbolos. Em [Kem11b] T. Kempton provou que para um potencial $f$ dependendo de finitas coordenadas sobre um shift enumerável com a propriedade BIP, $\mu_{\beta f}$ converge quando $\beta \rightarrow \infty$. Recentemente esse resultado foi estendido para shifts topologicamente mixing satisfazendo a condição $\sum_{i \in \mathbb{N}} e^{\left.\sup f\right|_{[i]}}<\infty$ (veja [VJ15]).

Por outro lado, são conhecidos exemplos de potencias Hölder contínuos(necessariamente dependendo de infinitas coordenadas) para os quais $\mu_{\beta f}$ não converge quando $\beta \rightarrow \infty$ ([CH10]). De fato, Ruszel e van Enter em [vEW07] mostraram que no caso de spin compacto mas contínuo ( $\mathbb{S}^{1}$ ) a sequência pode divergir mesmo o potencial dependendo de um número finito de símbolos (veja $\left[\mathrm{BCL}^{+} 11\right]$ para um review sobre este teorema). Usando estas ideias recentemente [CR14] construíram potenciais Lipschitz tais que $\left(\mu_{\beta f}\right)$ não converge quando $\beta \rightarrow \infty$, em shifts com um número finito de símbolos.

Em [JMU05] O. Jenkinson, R.D. Mauldin e M. Urbański provaram existência de pontos de acumulação da família de estados de equilíbrio $\left(\mu_{\beta f}\right)_{\beta}$ associados ao potential $f$ Hölder contínuo sobre um shift de Markov enumerável com a propriedades BIP. Além disso, mostraram que cada ponto de acumulação é uma medida maximizante para o potencial $f$. Para definirmos o que é uma medida maximizante precisamos do seguinte conceito:

Definição 14. Se o potencial $f \in C^{0}\left(\Sigma_{A}(\mathbb{N})\right)$ é limitado superiormente, definimos o valor maximizante por

$$
m(f)=\sup _{\mu \in \mathcal{M}_{\sigma}\left(\Sigma_{A}(\mathbb{N})\right)} \int f d \mu .
$$

Qualquer probabilidade $\sigma$-invariante atingindo este supremo é chamada maximizante (ou $f$ maximizante). Denota-se o conjunto de todas as medidas $f$-maximizantes por $\mathcal{M}_{\max }(f)$. 
No caso de um shift compacto e topologicamente mixing e um um potencial $f$ com variação somável, é sabido que qualquer ponto de acumulação (na topologia fraca*) da família de estados de equilíbrio $\left(\mu_{\beta f}\right)_{\beta}$ quando $\beta \rightarrow \infty$ é uma probabilidade $f$-maximizante.

Com efeito,

seja $\nu \in \mathcal{M}_{\sigma}\left(\Sigma_{A}(n)\right)$

$$
\beta \int f d \nu \leq h(\nu)+\beta \int f d \nu \leq h\left(\mu_{\beta f}\right)+\beta \int f d \mu_{\beta f} \leq h_{\mathrm{top}}(\sigma)+\beta \int f d \mu_{\beta f}
$$

se $\mu$ é ponto de acumulação, então $\int f d \nu \leq \int f d \mu \forall \nu \in \mathcal{M}_{\sigma}\left(\Sigma_{A}(n)\right)$.

Note que a existência de medidas maximizantes para o caso de um shift com um número finito de símbolos é consequência da compacidade de $\mathcal{M}_{\sigma}\left(\Sigma_{A}(n)\right)$ e a continuidade do mapa $\nu \rightarrow \int f d \nu$. Observe também, que na equação (2.20) foi fundamental o fato que $h_{\text {top }}(\sigma)$ é finita. No caso não compacto o argumento é mais elaborado, usa o Teorema de Prohorov, citamos aqui o resultado de O Jenkinson, R.D Mauldin e M. Urbański [JMU06]:

Teorema 9. Sejam $\left(\Sigma_{A}(\mathbb{N}), \sigma\right)$ um shift finitamente primitivo e $f$ um potencial com variação somável e satisfazendo $\sum_{i \in \mathbb{N}} \exp \left(\left.\sup f\right|_{[i]}\right)<\infty$. Então a familia de estados Gibbs $\left(\mu_{\beta f}\right)_{\beta}$ tem pelo menos um ponto de acumulação na topologia fraca* quando $\beta \rightarrow \infty$ e qualquer ponto de acumulação $\mu$ é uma medida maximizante.

Corolário 2. Seja $\Sigma_{A}(\mathbb{N})$ finitamente primitivo, $f$ com variação somável e $P(f)<\infty$. Suponha ademais que existe uma única medida maximizante $\mu$. Então $\mu_{\beta} \rightarrow \mu$ na topologia fraca*.

Demonstração. Suponha que $\mu_{\beta}$ não converge para $\mu$. Então existe $\epsilon_{0}>0$ tais que

$$
d\left(\mu_{\beta}, \mu\right)^{1} \geq \epsilon_{0} \quad \forall \beta>0 .
$$

Agora, usando que a sequência $\left(\mu_{\beta f}\right)_{\beta>0}$ é rígida (teorema acima) existe uma subsequência $\left(\mu_{\beta_{j}}\right)_{j}$ convergente, pelo teorema acima teríamos que $\mu_{\beta_{j}} \rightarrow \mu$ o qual contradiz (2.21).

I. Morris provou em [Mor] que qualquer ponto de acumulação de $\left(\mu_{\beta f}\right)_{\beta}$ tem entropia maximal entre as medidas maximizantes. Mais precisamente:

Teorema 10. Assuma $\left(\Sigma_{A}(\mathbb{N}), \sigma\right)$ finitamente primitivo e seja $f: \Sigma_{A}(\mathbb{N}) \rightarrow \mathbb{R}$ com variação somável e $P(f)<\infty$. Para cada $\beta>1$ seja $\mu_{\beta f}$ ó único estado Gibbs-equilíbrio associado a $\beta f$, $e$ seja $\mu$ qualquer ponto de acumulação de $\mu_{\beta f}$. Então

1. $h(\mu)=\lim _{\beta \rightarrow \infty} P(\beta f-\beta m(f))=\lim _{\beta \rightarrow \infty} h\left(\mu_{\beta f}\right)=\sup _{\nu \in \mathcal{M}_{\max }(f)} h(\nu)$.

2. $\lim _{\beta \rightarrow \infty} \frac{1}{\beta} P(\beta f)=m(f)$.

Além disso, Morris provou que existe um alfabeto $\mathcal{A} \subset \mathbb{N}$ finito tal que para qualquer ponto de acumulação $\mu$ de $\left(\mu_{\beta f}\right)_{\beta}$ temos que $\operatorname{supp}(\mu) \subset \Sigma_{A}(\mathcal{A})$. Ademais, esse mesmo resultado foi obtido para medidas maximizantes para potenciais coercivos para shifts BIP e transitivos respectivamente em [RG10, RJ14]. Ou seja, se o potencial é coercivo eles provaram a existência da medida maximizante e também que o suporte de qualquer maximizante está contido em um subshift compacto.

Sendo assim é natural investigar o comportamento de $\mu_{\beta}\left[x_{0} \ldots x_{n-1}\right]$ quando $\beta \rightarrow \infty$.

Pelos resultados acima, sabemos que se $x_{0}, \ldots, x_{n-1}$ foram grandes o suficiente e o potencial $f$ for coercivo então $\lim _{\beta \rightarrow \infty} \mu_{\beta}\left[x_{0} \ldots x_{n-1}\right]=0$.

\footnotetext{
${ }^{1}$ Como $\Sigma_{A}(\mathbb{N})$ é separável, definimos a seguinte métrica $d(\mu, \nu):=\inf \left\{\alpha>0: \mu(A) \leq \nu\left(A_{\alpha}\right)+\alpha\right.$ e $\nu(A) \leq$ $\left.\mu\left(A_{\alpha}\right)+\alpha \forall A \in \mathcal{B}\left(\Sigma_{A}(\mathbb{N})\right)\right\}$ (métrica de Levy-Prohorov). A topologia induzida por esta métrica coincide com a topologia fraca*.
} 
Queremos estudar: o possível limite

$$
\lim _{\beta \rightarrow \infty} \frac{1}{\beta} \log \mu_{\beta}\left[x_{0} \ldots x_{n-1}\right] .
$$

Ou seja, gostaríamos de descrever o comportamento da forma $\mu_{\beta}\left[x_{0} \ldots x_{n-1}\right] \cong e^{-\beta I}$, onde a função $I$ seria a velocidade na qual a sequência de medidas está convergindo. Uma das dificuldades em geral é achar a função taxa $I$. Neste trabalho usaremos argumentos de Otimização Ergódica para achar a função $I$, estendendo o resultado de A. Baraviera, A. Lopes e P. Thieullen [BLT06] que determinaram a função taxa no caso de um shift com um alfabeto finito . 


\section{Capítulo 3}

\section{Otimização Ergódica}

O problema principal em Otimização Ergódica é garantir a existência e descrever as medidas maximizantes do sistema. Depois das notas de Oliver Jenkinson (veja [Jen06]) o estudo de medidas maximizantes se tornou conhecido como Otimização Ergódica. Para uma referência mais recente veja [BLL13].

A principal conjectura da área até pouco tempo atrás era que para uma dinâmica espansora, genericamente no espaço de potenciais Lipschitz a medida maximizante é única e suportada numa órbita periódica. Depois de resultados preliminares [CLT01, QS12], a conjectura recentemente foi provada por G. Contreras em [Con14].

No contexto compacto, como já foi visto no capítulo anterior, a existência de medidas maximizantes é imediata. Sendo assim, o que se desenvolveu na área até então foram ferramentas para o estudo do suporte desta medidas e propriedades do conjunto das medidas maximizantes. Cabe destacar funções chamadas sub-ações, usadas para estudar o suporte de medidas maximizantes (ver [CLT01]). No contexto não compacto a existência de tais objetos mostrou-se ser equivalente a existência de medidas maximizantes [JMU06]. Além disso, o fato de existir uma sub-ação limitada implica que o suporte da medida maximizante, caso exista, está contido num subshift compacto (ver [RG10]).

Neste capítulo provaremos um resultado fundamental para o resto da tese, a existência de uma sub-ação calibrada limitada para o caso de um shift enumerável. Esta sub-ação calibrada é construída como ponto limite da sequência $\left(\frac{1}{\beta} \log h_{\beta}\right)$. Como corolário deste resultado obtemos uma nova prova para a existência de medidas maximizantes para o caso não compacto e BIP. Para shifts sem a propriedade BIP a única prova existente na atualidade é feita via aproximação por órbitas periódicas no caso de potencias de variação somável e coercivos [RJ14]. Nesse contexto de shifts transitivos sem a propriedade BIP, ainda não se sabe se existem sub-ações ou não mesmo na presença de medidas maximizantes. O resto do capítulo esta dedicado a estender para o caso de shifts enumeráveis as principais propriedades do Potencial de Mañé e da Barreira de Peierls. Estes objetos estão relacionados com o conjunto de Aubry. O conjunto de Aubry, como veremos a seguir, é o conjunto maximizante:

Definição 15. Dizemos que $\Omega \subseteq \Sigma_{A}(\mathbb{N})$ é um conjunto maximizante para $f$ quando: $\mu \in \mathcal{M}_{\sigma}\left(\Sigma_{A}(\mathbb{N})\right)$ é $f$-maximizante se, e somente se, $\operatorname{supp}(\mu) \subseteq \Omega$.

De fato, já existe uma construção do conjunto de Aubry para potencias localmente Hölder em shifts finitamente primitivos feita em [RG10]. No entanto, a construção neste artigo é feita via aproximação por compactos. Aqui apresentamos uma abordagem alternativa onde todos os objetos, sub-ações, Potencial de Mañé e a Barreira de Peierls estão definidas em todo o shift.

Antes de abordarmos provas da existência de medidas maximizantes, o seguinte exemplo mostra que $\mathcal{M}_{\max }(f)$ poder ser vazio no caso de um shift enumerável [JMU07] e potencial limitado. Esse exemplo em particular ilustra a importânça da coercividade do potencial se quisermos garantir a existência de maximizantes. 
Exemplo 2. Seja $\Sigma_{A}(\mathbb{N})=\mathbb{N}^{\mathbb{N}}$. Defina $f: \Sigma_{A}(\mathbb{N}) \rightarrow \mathbb{R}$ constante sobre 2-cilindros por:

$$
f[m, n]= \begin{cases}-\frac{1}{n(n+1)}, & \text { se } m=n+1 \\ -1, & \text { se } m \neq n+1\end{cases}
$$

Seja $\mu_{n}:=\frac{1}{n} \sum_{j=0}^{n-1} \delta_{\sigma^{j}\left(x^{(n)}\right)}$ a medida invariante suportada na órbita periódica gerada por

$$
\begin{gathered}
x^{(n)}=\overline{(n, n-1, \ldots, 1)} . \\
\int_{\Sigma_{A}(\mathbb{N})} f d \mu_{n}=\frac{1}{n} \sum_{j=0}^{n-1} f\left(\sigma^{j}\left(x^{(n)}\right)\right) \\
=-\frac{1}{n}\left[\sum_{j=0}^{n-2} \frac{1}{(n-j-1)(n-j)}\right]-\frac{1}{n} \\
=-\frac{1}{n}\left[\sum_{j=0}^{n-2} \frac{1}{n-j-1}-\frac{1}{n-j}\right]-\frac{1}{n} \\
=\frac{1-2 n}{n^{2}} . \\
m(f)=\sup _{\nu \in \mathcal{M}_{\sigma}} \int_{\Sigma_{A}(\mathbb{N})} f d \nu \geq \sup _{n \geq 1} \int_{\Sigma_{A}(\mathbb{N})} f d \mu_{n}=0 .
\end{gathered}
$$

Como $f<0$ então $\int_{\Sigma_{A}(\mathbb{N})} f d \nu<0$ para toda $\nu \in \mathcal{M}_{\sigma}\left(\Sigma_{A}(\mathbb{N})\right)$, implicando que $f$ não tem medida maximizante.

\subsection{Existência de maximizantes e sub-ações}

No caso de um shift compacto e uma função $f$ contínua a existência das medidas maximizantes é uma consequência imediata da compacidade de $\mathcal{M}_{\sigma}$ na topologia fraca*. No entanto, no caso não compacto ainda a existência de dita medida é um problema não trivial.

Nesta seção listaremos alguns trabalhos da área que garantem a existência de medidas maximizantes no caso de um shift enumerável. Destacamos também que nesta tese conseguimos (veja Teorema 15) garantir, de forma independente, que $\mathcal{M}_{\max }(f) \neq \emptyset$ no caso de potenciais de variação somável e pressão finita definidos em shifts finitamente primitivos. Nossa prova é consequência de termos construído uma sub-ação calibrada limitada.

O Jenkinson, R.D Mauldin e M. Urbański mostraram em [JMU06] que para um potencial $f$ com variação somável, definidos sobre um shift finitamente primitivo, existe uma medida maximizante. A prova segue os seguintes passos:

Definição 16. Para uma função contínua $T: \Sigma_{A}(\mathbb{N}) \rightarrow \Sigma_{A}(\mathbb{N})$, uma função da forma $\phi-\phi \circ T$, onde $\phi \in C_{b}\left(\Sigma_{A}(\mathbb{N})\right.$, é chamada função cobordo. Duas funções $f, g$ são chamadas cohomólogas se a diferença entre elas é dada por uma função cobordo.

Denotamos por $f \sim g$.

Note que se $f$ e $g$ são cohomólogas então para qualquer medida invariante $\mu$ temos que $\int f d \mu=\int g d \mu$.

Definição 17. Uma função contínua $\tilde{f} \sim f$ é chamada uma forma normal para $f$ se $\tilde{f}^{-1}(\sup \tilde{f}) \supset \operatorname{supp}(\nu)$, para alguma medida $\nu \sigma$-invariante. 
Teorema 11 (O Jenkinson, R.D Mauldin e M. Urbański, [JMU06]). Suponha $T: X \rightarrow X$ uma função contínua no espaço topológico $X$. Assuma que a função contínua $f: X \rightarrow \mathbb{R}$ tem uma forma normal $\tilde{f}$. Então

$$
\mathcal{M}_{\max }(f)=\left\{\nu \in \mathcal{M}_{\sigma}: \operatorname{supp}(\nu) \subset \tilde{f}^{-1}(\sup \tilde{f})\right\} \neq \emptyset .
$$

Teorema 12 (O Jenkinson, R.D Mauldin e M. Urbański, [JMU06]). Seja $\left(\Sigma_{A}(\mathbb{N}), \sigma\right)$ finitamente primitivo. Seja $f$ como variação somável, satisfazendo $\sum_{i \in \mathbb{N}} e^{\left.\sup f\right|_{[i]}}<\infty$. Então $f$ tem uma forma normal.

Ainda no contexto BIP R. Bissacot e E. Garibaldi provaram em [RG10] que, para um potencial $f$ localmente Hölder contínuo sobre um shift finitamente primitivo existe uma medida maximizante quando $f$ é coercivo, além disso, mostraram que o suporte de dita medida está contido em um subshift finito. No mesmo artigo introduziram pela primeira vez o conjunto de Aubry no contexto de shifts não compactos. Mais recentemente, R. Bissacot e R. Freire em [RJ14] provaram que para um potencial $f$ com variação somável e coercivo definido sobre um shift topologicamente transitivo (o qual é equivalente a dizer que $A$ é irredutível) existe uma medida maximizante suportada em um subshift finito. Mais precisamente:

Definição 18. Dizemos que A é irredutivel se, para quaisquer $i, j \in \mathbb{N}$ permitidos, existe uma palavra $w=\left(w_{1}, \ldots, w_{k}\right)$ tal que iwj é uma palavra permitida, ou seja, $A\left(i, w_{1}\right)=1, A\left(w_{i}, w_{i+1}\right)=1$ para $i=1, \ldots, k$ e $A\left(w_{k}, j\right)=1$.

Teorema 13 (R. Bissacot e R. Freire, [RJ14]). Sejam $\Sigma_{A}(\mathbb{N})$ um shift irredutivel e $f: \Sigma_{A}(\mathbb{N}) \rightarrow \mathbb{R}$ uma função com variação somável e coerciva. Então existe um subconjunto finito $\mathcal{A} \subset \mathbb{N}$ tais que $\left.A\right|_{\mathcal{A} \times \mathcal{A}}$ é irredutivel $e$

$$
\sup _{m \in \mathcal{M}_{\sigma}\left(\Sigma_{A}(\mathbb{N})\right)} \int f d m=\sup _{m \in \mathcal{M}_{\sigma}\left(\Sigma_{A}(\mathcal{A})\right)} \int f d m
$$

Além do mais

$$
\operatorname{supp}\left(\mu_{\max }\right) \subset \Sigma_{A}(\mathcal{A}) .
$$

Medidas maximizantes admitem objetos duais, conhecidos como sub-ações. Uma sub-ação, como veremos abaixo, fornece informação importante sobre o suporte das medidas de probabilidades maximizantes.

Existem varias formas de construir sub-ações, neste trabalho mostraremos, por exemplo, que qualquer ponto de acumulação de $\left(\frac{1}{\beta} \log h_{\beta}\right)$ na topologia de convergência em compactos, é uma subação calibrada quando o potencial $f$ for de variação somável, pressão finita e o shift for finitamente primitivo. Construída dita sub-ação nós provamos que existe uma medida $f$-maximizante para o caso de um shift enumerável, esta é uma nova prova para existência de medidas maximizantes, veja [RG10, RJ14, JMU07, JMU06, JMU05] onde aparecem outras provas conhecidas para o caso de um shift enumerável. É sabido também que para um potencial contínuo genérico (com respeito à topologia uniforme) sobre um shift finito, não há sub-ação contínua associada (veja [Mor07]). Para o leitor que deseje conhecer um pouco mais sobre sub-ações e como elas surgiram na Dinâmica Simbólica, aconselhamos ler a seção 1.4 de [Gar06].

Definição 19 (Sub-ação). Suponha $f: \Sigma_{A}(\mathbb{N}) \rightarrow \mathbb{R}$ é contínua e limitada superiormente. Uma sub-ação (para o potencial $f$ ) é uma função $V \in C^{0}\left(\Sigma_{A}(\mathbb{N})\right)$ que satisfaz

$$
V(x)+f(x)-m(f) \leq V(\sigma(x)), \quad \forall x \in \Sigma_{A}(\mathbb{N}) .
$$

Definição 20. Uma função contínua $V: \Sigma_{A}(\mathbb{N}) \rightarrow \mathbb{R}$ chama-se sub-ação calibrada para o potencial $f$ se satisfaz $(3.2)$ e se para qualquer $x \in \Sigma_{A}(\mathbb{N})$ existe $y \in \Sigma_{A}(\mathbb{N})$ tal que $\sigma(y)=x$ e

$$
V(x)=V(y)+f(y)-m(f) .
$$


Uma das características mais importantes das sub-ações consiste na localização dos suportes das probabilidades maximizantes. Isto é, seja $V: \Sigma_{A}(\mathbb{N}) \rightarrow \mathbb{R}$ uma sub-ação limitada, provaremos que

$$
\mathcal{M}_{\max }(f)=\left\{\mu \in \mathcal{M}_{\sigma}\left(\Sigma_{A}(\mathbb{N})\right): \operatorname{supp}(\mu) \subset(f+V-V \circ \sigma)^{-1}(m(f))\right\} .
$$

Com efeito, defina $g:=f+V-V \circ \sigma-m(f)$ e observe que

$$
g \leq 0 \text { e } \int g d \mu=0 \forall \mu \in \mathcal{M}_{\max }(f) .
$$

Assim, quando $\mu$ é uma medida $f$-maximizante, $g \equiv 0 \mu$ q.t.p, isto é

$$
\operatorname{supp}(\mu) \subset g^{-1}(0)=(f+V-V \circ \sigma)^{-1}(m(f)) .
$$

Reciprocamente, cada medida $\mu \sigma$-invariante tal que $\operatorname{supp}(\mu) \subset(f+V-V \circ \sigma)^{-1}(m(f))$ é uma medida $f$-maximizante.

O seguinte operador foi introduzido por Bousch [Bou01] para estudar medidas maximizantes no caso de um shift compacto, posteriormente adaptado para shifts enumeráveis por O. Jenkinson, R. D. Mauldin e M. Urbański e utilizado na construção de uma sub-ação calibrada:

Definição 21 (Operador de Lax-Oleinik). Seja $\sigma: \Sigma_{A}(\mathbb{N}) \rightarrow \Sigma_{A}(\mathbb{N})$ sobrejetora, e $f: \Sigma_{A}(\mathbb{N}) \rightarrow \mathbb{R}$ qualquer função. Se $\varphi: \Sigma_{A}(\mathbb{N}) \rightarrow \mathbb{R}$ então para cada $x \in \Sigma_{A}(\mathbb{N})$, define $M_{f} \varphi(x) \in(-\infty, \infty]$ por

$$
M_{f} \varphi(x):=\sup _{y \in \sigma^{-1} x}(f+\varphi)(y) .
$$

Em [RG10] R. Bissacot e E. Garibaldi constroem uma sub-ação (não calibrada) limitada. Em ambos casos a existência de uma sub-ação foi fundamental para provar a existência de medidas maximizantes no caso de um shift enumerável. Na proposição 6(f) e o Teorema 14 apresentamos novas construções de sub-ações calibradas para o caso de um shift enumerável.

O seguinte exemplo construído por O. Jenkinson, R. D. Mauldin e M. Urbański mostra que $M_{f, \phi}$ poder ser descontínuo mesmo quando $f$ e $\phi$ contínuos.

Exemplo 3. Seja $T$ o mapa contínuo linear por partes $T:[0,1] \rightarrow[0,1]$ determinado pelos pontos $T(0)=0, T(1 / 4)=1 / 4, T(3 / 4)=1 / 4, T(1)=1$.

$$
\begin{array}{r}
M_{0, \phi}(x)=\max _{T y=x} \phi(y) . \\
M_{0, \phi}(0)=0, M_{0, \phi}(1 / 4)=1, M_{0, \phi}(1 / 2)=1, M_{0, \phi}(1)=1 .
\end{array}
$$

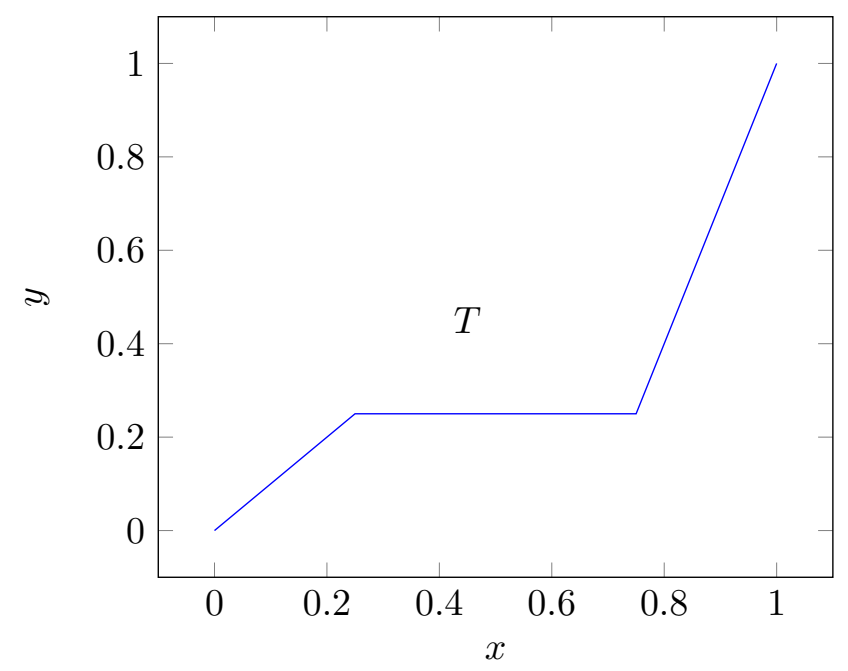



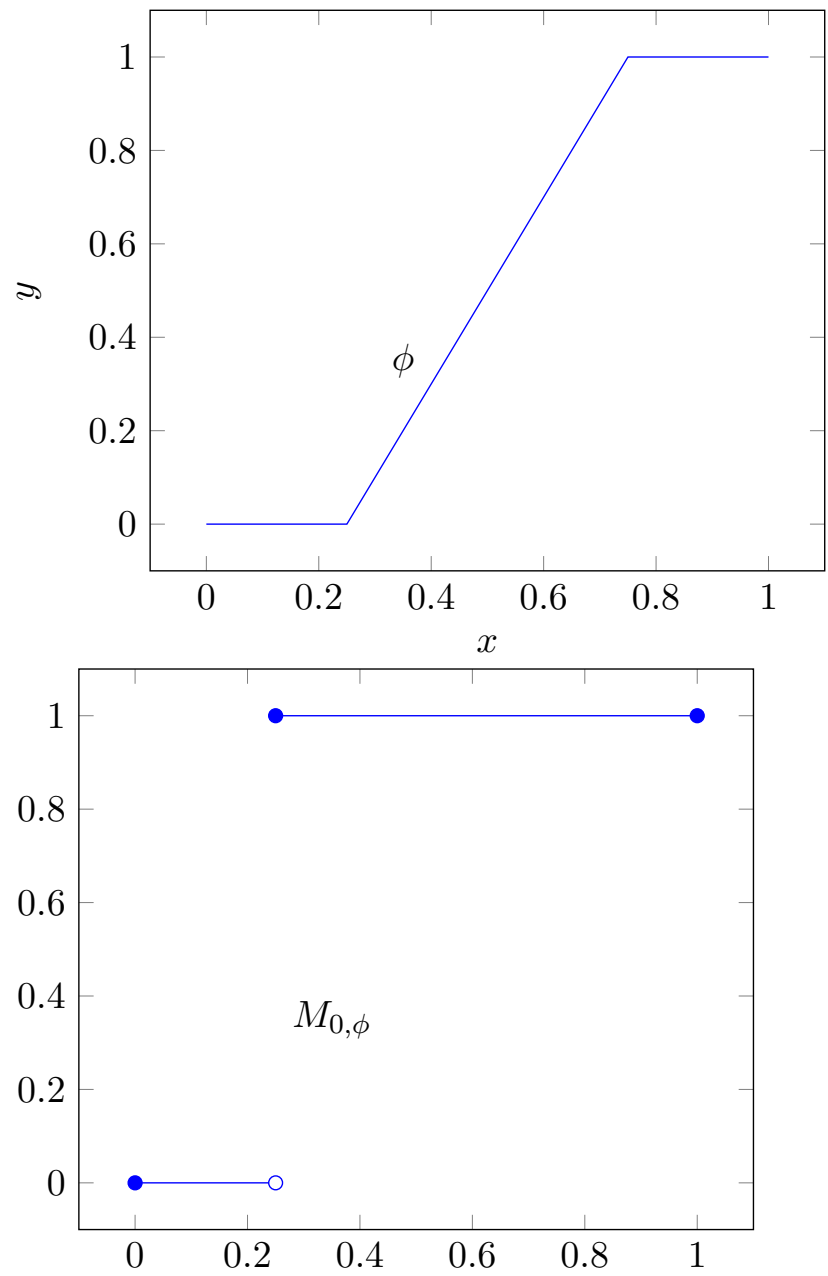

O objetivo principal deste capítulo é provar que qualquer ponto limite da sequência $\left(\frac{1}{\beta} \log h_{\beta}\right)$ é uma sub-ação calibrada limitada com variação somável. Para atingir dito objetivo precisamos de alguns lemas técnicos:

Lema 8. A sequência $\varphi_{\beta}=\frac{1}{\beta} \log h_{\beta}$ é equicontínua e uniformemente limitada.

Demonstração. Sejam $\epsilon>0$ e $x, y \in \Sigma_{A}(\mathbb{N})$ tais que $d(x, y) \leq r^{m}, m \geq 1$. Então usando um argumento similar à prova da Proposição 1 afirmamos que para todo $n \geq 1$

$$
\frac{h_{\beta}(x)}{h_{\beta}(y)} \leq e^{\beta\left(\operatorname{Var}_{n+m}(f)+\cdots+\operatorname{Var}_{m+1}(f)\right)},
$$

Observe que provar (3.6) é equivalente a provar a seguinte desigualdade (veja equação (2.7))

$$
e^{\beta S_{n} f(p x)-\beta S_{n} f(p y)}-e^{\beta\left(\operatorname{Var}_{n+m}(f)+\cdots+\operatorname{Var}_{m+1}(f)\right)} \leq 0 .
$$

e esta última desigualdade é verdadeira pois

$$
\begin{aligned}
S_{n} f(p x)-S_{n} f(p y) & =\left[f\left(p_{0} \ldots p_{n-1} x\right)-f\left(p_{0} \ldots p_{n-1} y\right)\right]+\cdots+\left[f\left(p_{n-1} x\right)-f\left(p_{n-1} y\right)\right] \\
& \left.\leq \operatorname{Var}_{n+m}(f)+\cdots+\operatorname{Var}_{m+1}(f)\right) .
\end{aligned}
$$

Portanto, da equação (3.6) segue que:

$$
\frac{1}{\beta} \log h_{\beta}(x)-\frac{1}{\beta} \log h_{\beta}(y) \leq \operatorname{Var}_{n+m}(f)+\cdots+\operatorname{Var}_{m+1}(f) .
$$

Assim

$$
\varphi_{\beta}(x)-\varphi_{\beta}(y) \leq \operatorname{Var}_{n+m}(f)+\cdots+\operatorname{Var}_{m+1}(f) \rightarrow 0(m \rightarrow \infty) .
$$


$\varphi_{\beta}$ é uniformemente limitada, pois usando a Proposição 3 sabemos que para todo $x \in \Sigma_{A}(\mathbb{N})$, $e^{\beta C_{1}} \leq h_{\beta}(x) \leq e^{\beta C_{2}}$.

Observação 3. Como $\Sigma_{A}(\mathbb{N})$ é um espaço separável, existe $X \subset \Sigma_{A}(\mathbb{N})$ subconjunto denso e enumerável. Digamos $X=\left\{x_{1}, x_{2}, \ldots\right\}$. A sequência $\left(V_{\beta_{n}}\left(x_{1}\right)\right)_{n \in \mathbb{N}}$ é equicontínua e uniformemente limitada, assim usando a Proposiçẫo 15, possui uma subsequência convergente. Desta forma obtemos um subconjunto infinito $\mathbb{N}_{1} \subset \mathbb{N}$ tal que o limite $\lim _{n \in \mathbb{N}_{1}} V_{\beta_{n}}\left(x_{1}\right)=a_{1}$ existe. Também a sequência $\left(V_{\beta_{n}}\left(x_{2}\right)\right)_{n \in \mathbb{N}_{1}}$ é eqüicontinua e uniformemente limitada, assim podemos achar um conjunto infinito $\mathbb{N}_{2} \subset \mathbb{N}_{1}$ tal que o limite $\lim _{n \in \mathbb{N}_{2}} V_{\beta_{n}}\left(x_{2}\right)=a_{2}$ existe. Prosseguindo analogamente, conseguimos, para cada $i \in \mathbb{N}$, um subconjunto infinito $\mathbb{N}_{i} \subset \mathbb{N}$, tal que $\mathbb{N}_{1} \supset \mathbb{N}_{2} \supset \cdots \supset \mathbb{N}_{i} \supset \cdots$ e para cada $i$, existe o limite $\lim _{n \in \mathbb{N}_{i}} V_{\beta_{n}}\left(x_{i}\right)=a_{i}$. Definimos então um subconjunto infinito $\mathbb{N}^{*} \subset \mathbb{N}$ tomando como $i$-ésimo elemento de $\mathbb{N}^{*}$ o $i$-ésimo elemento de $\mathbb{N}_{i}$. Desta maneira, para cada $i \in \mathbb{N}$ a sequência $\left(V_{\beta_{n}}\left(x_{i}\right)\right)_{n \in \mathbb{N}^{*}}$ é a partir do seu i-ésimo elemento, uma subsequência de $\left(V_{\beta_{n}}\left(x_{i}\right)\right)_{n \in \mathbb{N}_{i}}$ e, portanto, converge. Isto prova que a subsequência $\left(V_{\beta_{n}}\right)_{n \in \mathbb{N}^{*}}$ converge em cada ponto $x_{i} \in X$.

Usando a observação acima e a Proposição 15 concluímos que existe uma subsequência $\left(V_{\beta_{n}}\right)$ que converge uniformemente em qualquer subconjunto compacto $K$ de $\Sigma_{A}(\mathbb{N})$.

Lema 9. Seja $V: \Sigma_{A}(\mathbb{N}) \rightarrow \mathbb{R}$ definida por $V(x):=\lim _{\beta_{i} \rightarrow \infty} \frac{1}{\beta_{i}} \log h_{\beta_{i}}(x)$. Então $V$ tem variação somável.

Demonstração. Sejam $x, y \in \Sigma_{A}(\mathbb{N}) \operatorname{com} d(x, y) \leq r^{m}, m \geq 1$ afirmamos que para todo $n \geq 1$ temos

$$
h_{\beta_{i}}(x) \leq h_{\beta_{i}}(y) e^{\beta_{i}\left(\operatorname{Var}_{n+m}(f)+\cdots+\operatorname{Var}_{m+1}(f)\right)} .
$$

Observe que provar (3.7) é equivalente a provar a seguinte desigualdade (veja equação (2.7))

$$
e^{\beta_{i} S_{n} f(p x)-\beta_{i} S_{n} f(p y)}-e^{\beta_{i}\left(\operatorname{Var}_{n+m}(f)+\cdots+\operatorname{Var}_{m+1}(f)\right)} \leq 0 .
$$

e esta última desigualdade é verdadeira pois

$$
\begin{aligned}
S_{n} f(p x)-S_{n} f(p y) & =\left[f\left(p_{0} \ldots p_{n-1} x\right)-f\left(p_{0} \ldots p_{n-1} y\right)\right]+\cdots+\left[f\left(p_{n-1} x\right)-f\left(p_{n-1} y\right)\right] \\
& \left.\leq \operatorname{Var}_{n+m}(f)+\cdots+\operatorname{Var}_{m+1}(f)\right) .
\end{aligned}
$$

Deste modo, da equação (3.7) temos que:

$$
\frac{1}{\beta_{i}}\left(\log h_{\beta_{i}}(x)-\log h_{\beta_{i}}(y)\right) \leq \operatorname{Var}_{n+m}(f)+\cdots+\operatorname{Var}_{m+1}(f),
$$

fazendo $\beta_{i} \rightarrow \infty$ obtemos que

$$
V(x)-V(y) \leq \operatorname{Var}_{n+m}(f)+\cdots+\operatorname{Var}_{m+1}(f),
$$

assim

$$
\operatorname{Var}_{m}(V) \leq \operatorname{Var}_{n+m}(f)+\cdots+\operatorname{Var}_{m+1}(f)
$$

portanto

$$
\operatorname{Var}(V)=\sum_{m=1}^{\infty} \operatorname{Var}_{m}(V) \leq \sum_{m=1}^{\infty}\left(\operatorname{Var}_{n+m}(f)+\cdots+\operatorname{Var}_{m+1}(f)\right)<\infty .
$$

Assim concluímos que $V$ tem variação somável.

Neste ponto estamos prontos para provar o principal resultado deste capítulo, este é: 
Teorema 14. Seja $\left(\Sigma_{A}(\mathbb{N}), \sigma\right)$ um shift finitamente primitivo e $f$ uma função com variação somável e $P(f)<\infty$. Então a função $V(x):=\lim _{\beta_{i} \rightarrow \infty} \frac{1}{\beta_{i}} \log h_{\beta_{i}}(x)$ é uma sub-ação calibrada limitada para o potencial $f$.

Demonstração. Da Observação acima sabemos que, existe uma subsequência tal que $\frac{1}{\beta_{n}} \log h_{\beta_{n}}$ converge uniformemente em cada compacto $K \subset \Sigma_{A}(\mathbb{N})$, isto é

$$
\lim _{n \rightarrow \infty} \frac{1}{\beta_{n}} \log h_{\beta_{n}}(x):=V(x) .
$$

abusando da notação escreveremos

$$
\lim _{\beta \rightarrow \infty} \frac{1}{\beta} \log h_{\beta}(x):=V(x) .
$$

Note que pela proposição 3 temos que $V$ é limitada e $C_{1} \leq V(x) \leq C_{2}, \forall x \in \Sigma_{A}(\mathbb{N})$.

Seja $x \in \Sigma_{A}(\mathbb{N})$ fixo. Como $\left.\lim _{j \rightarrow \infty} \sup f\right|_{[j]}=-\infty$ (veja o Lema 4 ), existe $j_{1}:=j_{1}(x)$ suficientemente grande tal que para $\forall j \geq j_{1}$,

$$
f(j x)<f(0 x)+C_{1}-C_{2}-1 .
$$

Onde $C_{1}$ e $C_{2}$ são as constantes da Proposição 3.

Por outro lado como $\sum_{j=0}^{\infty} e^{f(j x)}<\infty$ (veja o Lema 4), então existe $j_{0}:=j_{0}(x)$ suficientemente grande, sem perda de generalidade suponha $j_{0}>j_{1}$, tal que

$$
\sum_{j=j_{0}+1}^{\infty} e^{f(i x)}<1 .
$$

Usando estas desigualdades vamos verificar que

$$
\sup _{j \in \mathbb{N}}(f(j x)+V(j x))=\max _{0 \leq j \leq j_{1}-1}(f(j x)+V(j x))=: \alpha(x)=\alpha .
$$

Com efeito, seja $j \geq j_{1}$, pela proposição 3 e a equação (3.10) segue que

$$
V(j x)-V(0 x) \leq C_{2}-C_{1} \leq f(0 x)-f(j x)-1,
$$

assim obtemos

$$
f(j x)+C_{2} \leq f(0 x)+C_{1}-1
$$

$\mathrm{e}$

$$
V(j x)+f(j x) \leq f(0 x)+V(0 x)-1<f(0 x)+V(0 x), \forall j \geq j_{1} .
$$

Logo (3.12) é satisfeita.

Note também que usando proposição 3 temos que

$$
f(0 x)+C_{1}-1 \leq f(0 x)+V(0 x)-1 \leq \alpha-1 .
$$

Note ainda que, sem perda de generalidade, usando o fato que $V$ é limitada podemos tomar $j_{0}$ grande o suficiente de modo que

$$
f(j x)+V(j x) \leq \alpha-1, \forall j \geq j_{0} .
$$


Agora escrevemos

$$
\sum_{j=0}^{\infty} e^{\beta f(j x)} h_{\beta}(j x)=\sum_{j=0}^{j_{1}-1} e^{\beta f(j x)} h_{\beta}(j x)+\sum_{j=j_{1}}^{j_{0}-1} e^{\beta f(j x)} h_{\beta}(j x)+\sum_{j=j_{0}}^{\infty} e^{\beta f(j x)} h_{\beta}(j x) .
$$

Da equação (3.9) podemos escrever $h_{\beta}(x)=e^{\beta\left(V(x)+\epsilon_{\beta}(x)\right)}$, aqui $\epsilon_{\beta}(x):=\frac{1}{\beta} \log h_{\beta}(x)-V(x)$ $\operatorname{com} \lim _{\beta \rightarrow \infty}\left|\epsilon_{\beta}\right|=0$. Assim resulta

$$
\sum_{j=0}^{j_{1}-1} e^{\beta f(j x)} h_{\beta}(j x)=\sum_{j=0}^{j_{1}-1} e^{\beta f(j x)} e^{\beta\left(V(j x)+\epsilon_{\beta}(j x)\right)} \leq j_{1} e^{\alpha \beta+\beta\left|\epsilon_{\beta}\right|} .
$$

Por outro lado, pela proposição 3, as equações (3.13) e (3.15) temos que

$$
\begin{aligned}
\sum_{j=j_{1}}^{j_{0}-1} e^{\beta f(j x)} h_{\beta}(j x) & \leq \sum_{j=j_{1}}^{j_{0}-1} e^{\beta\left(f(j x)+C_{2}\right)} \\
& \leq \sum_{j=j_{1}}^{j_{0}-1} e^{\beta\left(f(0 x)+C_{1}-1\right)} \\
& \leq\left(j_{0}-j_{1}\right) e^{\alpha \beta-\beta},
\end{aligned}
$$

usando a equação (3.16) resulta que

$$
\begin{aligned}
\sum_{j=j_{0}+1}^{\infty} e^{\beta f(j x)} h_{\beta}(j x) & =\sum_{j=j_{0}}^{\infty} e^{\beta(f(j x)+V(j x))+\beta \epsilon_{\beta}(j x)} \\
& =\sum_{j=j_{0}}^{\infty} e^{f(j x)+(\beta-1)[f(j x)+V(j x)]+V(j x)+\beta \epsilon_{\beta}(j x)} \\
& \leq\left(\sum_{j=j_{0}}^{\infty} e^{f(j x)}\right) e^{(\beta-1)(\alpha-1)+C_{2}+\beta \epsilon_{\beta}(j x)} \\
& <1 \cdot e^{(\beta-1)(\alpha-1)+C_{2}+\beta\left|\epsilon_{\beta}\right|} \text { (veja equação (3.11)). }
\end{aligned}
$$

Desta forma obtemos que

$$
\begin{aligned}
\sum_{j=0}^{\infty} e^{\beta f(j x)} h_{\beta}(j x) & \leq j_{1} e^{\alpha \beta+\beta\left|\epsilon_{\beta}\right|}+\left(j_{0}-j_{1}\right) e^{\alpha \beta-\beta}+e^{(\beta-1)(\alpha-1)+C_{2}+\beta\left|\epsilon_{\beta}\right|} \\
& =j_{1} e^{\alpha \beta+\beta\left|\epsilon_{\beta}\right|}\left[1+\left(\frac{j_{0}-j_{1}}{j_{1}}\right) e^{-\beta-\beta\left|\epsilon_{\beta}\right|}+\frac{1}{j_{1}} e^{-\beta-\alpha+C_{2}+1}\right]
\end{aligned}
$$

Colocando

$$
\bar{\varepsilon}(\beta):=\left(\frac{j_{0}-j_{1}}{j_{1}}\right) e^{-\beta\left(1+\left|\epsilon_{\beta}\right|\right)}+\frac{1}{j_{1}} e^{-\beta-\alpha+1+C_{2}},
$$

vemos que $\bar{\varepsilon}(\beta) \rightarrow 0$ quando $\beta \rightarrow \infty$. Portanto temos que

$$
\sum_{j=0}^{\infty} e^{\beta f(j x)} h_{\beta}(j x) \leq j_{1} e^{\alpha \beta+\beta\left|\epsilon_{\beta}\right|}(1+\bar{\varepsilon}(\beta)),
$$

assim

$$
\frac{1}{\beta} \log \sum_{j=0}^{\infty} e^{\beta f(j x)} h_{\beta}(j x) \leq \frac{1}{\beta} \log j_{1}+\frac{1}{\beta} \log (1+\bar{\varepsilon}(\beta))+\alpha+\left|\epsilon_{\beta}\right|
$$


logo usando o fato que $\lim _{\beta \rightarrow \infty} \frac{P(\beta f)}{\beta}=m(f)$ (veja teorema 10) obtemos que

$$
m(f)+V(x)=\lim _{\beta \rightarrow \infty} \frac{1}{\beta} \log \left(\lambda_{\beta} \cdot h_{\beta}(x)\right)=\lim _{\beta \rightarrow \infty} \frac{1}{\beta} \log \sum_{j=0}^{\infty} e^{\beta f(j x)} h_{\beta}(j x) \leq \alpha .
$$

Para provar a outra desigualdade, note que se $(f+V)(\bar{j} x)=\max _{0 \leq j \leq j_{1}-1}(f+V)(j x)$, para algum $0 \leq \bar{j} \leq j_{1}-1$ então

$$
\sum_{i=0}^{\infty} e^{\beta f(i x)} h_{\beta}(i x) \geq e^{\beta f(\bar{j} x)} h_{\beta}(\bar{j} x)
$$

assim

$$
\frac{1}{\beta} \log \sum_{i=0}^{\infty} e^{\beta f(i x)} h_{\beta}(i x) \geq f(\bar{j} x)+\frac{1}{\beta} \log h_{\beta}(\bar{j} x),
$$

deste modo

$$
\lim _{\beta \rightarrow \infty} \frac{1}{\beta} \log \sum_{i=0}^{\infty} e^{\beta f(i x)} h_{\beta}(i x) \geq f(\bar{j} x)+V(\bar{j} x)=\max _{0 \leq j \leq j_{1}-1}(f+V)(j x) .
$$

Assim concluímos que

$$
m(f)+V(x)=\max _{0 \leq j \leq j_{1}-1}(f+V)(j x)=\sup _{j \in \mathbb{N}}(f+V)(j x) .
$$

Em consequência $V$ é uma sub-ação calibrada.

Como corolário do teorema acima obtemos uma nova prova do seguinte resultado já provado por O. Jenkinson, R.D. Mauldin e M. Urbański em [JMU06, JMU05].

Teorema 15. Seja $\left(\Sigma_{A}(\mathbb{N}), \sigma\right)$ um shift finitamente primitivo. Seja $f: \Sigma_{A}(\mathbb{N}) \rightarrow \mathbb{R}$ com variação somável e $P(f)<\infty$ então $\mathcal{M}_{\max (f)} \neq \emptyset$.

Demonstração. Seja $R:=f+V-V \circ \sigma-m(f)$. Onde $V$ é a sub-ação calibrada que existe pelo teorema acima. Por definição de sub-ação calibrada temos que $R \leq 0$. Dado $x_{0} \in \Sigma_{A}(\mathbb{F})$, considere uma sequência de pontos $x_{n}$ tais que $\sigma\left(x_{n}\right)=x_{n-1}$ e $R\left(x_{n}\right)=0, n=1,2, \ldots$ (esta sequência existe pois $V$ é calibrada). Defina uma sequência de probabilidades $\nu_{n}:=\frac{1}{n}\left(\delta_{x_{1}}+\cdots+\delta_{x_{n}}\right)$. Usando o fato que $V$ é limitada e $f$ é coerciva, existe um conjunto finito $B \subset \mathbb{N}$ com $\mathbb{F} \subset B$, tais que qualquer $x_{n}$ é da forma $b_{n} \ldots b_{1} x_{0}, b_{i} \in B, n \geq 1$. Isto mostra que existe ao menos uma subsequência $\left(\nu_{n_{k}}^{*}\right)_{k}$ de $\left(\nu_{n}\right)_{n}$ convergindo para uma medida $\nu^{*} \in \mathcal{M}_{\sigma}\left(\Sigma_{A}(B)\right)$, pois $\mathcal{M}_{\sigma}\left(\Sigma_{A}(B)\right)$ é compacto. Assim, se $\left(\nu_{n_{k}}\right)_{k}$ é a extensão natural de $\left(\nu_{n_{k}}^{*}\right)_{k}$ para $\Sigma_{A}(\mathbb{N}), \log 0 \nu_{n_{k}} \rightarrow \nu \in \mathcal{M}_{\sigma}\left(\Sigma_{A}(\mathbb{N})\right)$ na topologia fraca*.

Afirmação 1. $\nu$ é uma medida $\sigma$-invariante e $\int R d \nu=0$.

Com efeito, seja $g \in C_{b}\left(\Sigma_{A}(\mathbb{N})\right)$, então

$$
\begin{aligned}
\left|\int g d \nu-\int(g \circ \sigma) d \nu\right| & =\lim _{n \rightarrow \infty}\left|\int g d \nu_{n}-\int g \circ \sigma d \nu_{n}\right| \\
& \left|\frac{1}{n} \sum_{j=0}^{n-1} g\left(\sigma^{j}(x)\right)-\frac{1}{n} \sum_{j=0}^{n-1} g\left(\sigma^{j+1}(x)\right)\right| \\
& =\frac{1}{n}\left|g(x)-g\left(\sigma^{n}(x)\right)\right| \leq \frac{2\|g\|_{\infty}}{n} .
\end{aligned}
$$

Portanto

$$
\int g d \nu=\int(g \circ \sigma) d \nu, \quad \forall g \in C_{b}\left(\Sigma_{A}(\mathbb{N})\right)
$$


Vejamos agora que $\int R d \nu=0$. Para $k \geq 1$ considere uma sequência de funções contínuas e limitadas dada por $R_{k}:=\max (R,-k)$, aqui $R_{k} \searrow R$ e $R_{k}\left(x_{n}\right)=0$ para todo $k \geq 1$ e $n \geq 1$. Assim

$$
\int R_{k} d \nu_{n}=\frac{1}{n}\left(R_{k}\left(x_{n}\right)+\cdots+R_{k}\left(x_{1}\right)\right)=0 .
$$

Logo

$$
0=\lim _{n \rightarrow \infty} \int R_{k} d \nu_{n}=\int R_{k} d \nu
$$

E usando o teorema da convergência monótona concluímos que

$$
\lim _{k \rightarrow \infty} \int R_{k} d \nu=\int R d \nu=0
$$

Assim provamos que existe uma medida invariante $\nu$ tais que $\int R d \nu=0$, desta forma $m(f)=\int f d \nu$. Concluindo assim que $\mathcal{M}_{\max (f)} \neq \emptyset$.

\subsection{Barreira de Peierls e Potencial de Mañé}

Nesta seção estendemos para o caso de um shift enumerável as principais propriedades do Potencial de Mañé e a Barreira de Peierls já conhecidas para o caso de um shift compacto, veja por exemplo [CLT01, GL08, GLT09].

O potencial de Mañé e a Barreira de Peierls são conceitos inspirados por noções similares em Teoria de Aubry-Mather para sistemas Lagrangianos (ver [CI99, Mañ96]). Foram A. Lopes, G. Contreras e P. Thieullen que trouxeram estes conceitos para à dinâmica simbólica (ver [CLT01]).

Nesta seção veremos também a importância do conjunto Aubry. Com ajuda do Potencial de Mañé podemos encontrar uma caracterização importante para as medidas maximizantes (veja proposição 6, (h)). Outro aspecto importante da Barreira de Peierls e do Potencial de Mañé é que com estes podemos provar que duas sub-ações calibradas diferentes são iguais modulo uma constante. Fato que terá muita importância no capítulo 3 , no momento de definir a função taxa $I$.

Definição 22. Dado um potencial $f \in C^{0}\left(\Sigma_{A}(\mathbb{N})\right)$, chamamos $x \in \Sigma_{A}(\mathbb{N})$ um ponto de Aubry se para todo $\epsilon>0$, existe $k \geq 1$ e $z \in \Sigma_{A}(\mathbb{N})$ tais que $d(x, z)<\epsilon, \sigma^{k}(z)=x$ e $\left|S_{k}(f-m(f))(z)\right|<\epsilon$. Denotamos por $\Omega(f, \sigma)$ o conjunto de Aubry, isto é, o conjunto de todos os pontos de Aubry.

Observação 4. $\Omega(f, \sigma) \neq \emptyset$ (veja afirmação 4).

Observação 5. Seja $f$ uma função com variação somável, na definição acima, veremos que a condiçãao $\sigma^{k}(z)=x$ é equivalente a $d\left(\sigma^{k}(z), x\right)<\epsilon$. Para verificar isso, chamamos de 1 à definição acima e definição 2 usando $d\left(\sigma^{k}(z), x\right)<\epsilon$, em vez de $\sigma^{k}(z)=x$, e provaremos que são equivalentes.

É claro que a definição 1 implica definição 2. Então resta provar que a definição 2 implica definição 1 .

Com efeito: Seja $x \in \Omega(f, \sigma)$ então dado $\epsilon>0 \exists n \geq 1 e$

$$
z=\left(x_{0}, \ldots, x_{l-1}, z_{l}, \ldots, z_{n-1}, x_{0}, x_{1}, \ldots, x_{k-1}, z_{n+k+1}, \ldots\right)
$$

tal que

$$
d(x, z)<\epsilon, d\left(x, \sigma^{n}(z)\right)<\epsilon e \quad\left|S_{n}(f-m(f))(z)\right|<\epsilon .
$$

Seja $w=\left(x_{0}, \ldots, x_{l-1}, z_{l}, \ldots, z_{n-1}, x_{0}, x_{1}, \ldots\right)$. A sequência $w$ satisfaz $d(x, w)<\epsilon, \sigma^{n}(w)=x$, 
e

$$
\begin{aligned}
\left|S_{n}(f-m(f))(w)\right| & =\left|\sum_{j=0}^{n-1}(f-m(f))\left(\sigma^{j}(w)\right)\right| \\
& =\left|(f-m(f))(w)+(f-m(f))(\sigma(w))+\cdots+(f-m(f))\left(\sigma^{n-1}(w)\right)\right| \\
& \leq\left|S_{n}(f-m(f))(z)\right|+|f(w)-f(z)|+\cdots+\left|f\left(\sigma^{n-1}(w)\right)-f\left(\sigma^{n-1}(z)\right)\right| \\
& \leq \epsilon+\operatorname{Var}_{n+k}(f)+\cdots+\operatorname{Var}_{1+k}(f)
\end{aligned}
$$

fazendo $k \rightarrow \infty$ obtemos o resultado requerido.

Afirmação 2. Dada $f$ com variação somável, $\Omega(f, \sigma)$ é $\sigma$-invariante, isto é, se $x \in \Omega(f, \sigma)$ então $\sigma(x) \in \Omega(f, \sigma)$.

Demonstração. Suponha que $x \in \Omega(f, \sigma)$ então dado $\epsilon>0$, existe um inteiro $k \geq 1$ e um ponto $z \in \Sigma_{A}(\mathbb{N})$ tal que $d(x, z)<\epsilon \leq r^{l}$ (para algum $\left.l>0\right), \sigma^{k}(z)=x$ e $\left|S_{k}(f-m(f))(z)\right|<\epsilon$. Defina $w:=\sigma(z) \operatorname{assim} w$ cumpre que $d(\sigma(x), w)<\epsilon, \sigma^{k}(w)=\sigma(x)$ e

$$
\begin{aligned}
\left|S_{k}(f-m(f))(w)\right| & =\left|f(\sigma(z))+\cdots+f\left(\sigma^{k}(z)\right)-k m(f)\right| \\
& \leq\left|S_{k}(f-m(f))(z)\right|+\left|f\left(\sigma^{k}(z)\right)-f(z)\right| \\
& =\left|S_{k}(f-m(f))(z)\right|+|f(x)-f(z)| \\
& <\epsilon+\operatorname{Var}_{l}(f),
\end{aligned}
$$

fazendo $l \rightarrow \infty$ concluímos que $\sigma(x) \in \Omega(f, \sigma)$.

Note que, da Proposição 6,(h) segue que o suporte de qualquer medida $f$-maximizante está contido no Aubry.

Agora, para verificar que qualquer medida invariante suportada no Aubry é $f$-maximizante, é suficiente provar o seguinte lema (ver equação 3.4):

Lema 10. $\Omega(f, \sigma) \subset(f+V-V \circ \sigma)^{-1}(m(f))$, aqui $V$ é uma sub-ação calibrada com variação somável.

Demonstração. Fixado $\delta>0$, tomamos $\epsilon \in(0, \delta)$. Seja $x \in \Omega(f, \delta)$, então existe $z \in \Sigma_{A}(\mathbb{N})$ e $k \in \mathbb{N}$ tal que

$$
d(x, z)<\epsilon, \quad \sigma^{k}(z)=x \quad \text { e }\left|S_{k}(f-m(f))(z)\right|<\epsilon .
$$

Ademais como $V$ é uniformemente contínua temos que

$$
|V(z)-V(x)|<\delta \text { sempre que } d(x, z)<\epsilon
$$

Observe agora que

$$
\begin{aligned}
0 \geq S_{k}(f+V-V \circ \sigma-m(f))(z) & =S_{k}(f-m(f))(z)+V(z)-V\left(\sigma^{k}(z)\right) \\
& >-\epsilon-\delta
\end{aligned}
$$

donde $\lim _{\delta \rightarrow 0} S_{k}(f+V-V \circ \sigma-m(f))(z)=0$. Em particular

$$
f(x)+V(x)-V(\sigma(x))-m(f)=\lim _{\delta \rightarrow 0}(f+V-V \circ \sigma-m(f))(z)=0 .
$$

Portanto

$$
x \in(f+V-V \circ \sigma)^{-1}(m(f)) .
$$

Logo

$$
\Omega(f, \sigma) \subset(f+V-V \circ \sigma)^{-1}(m(f)) .
$$




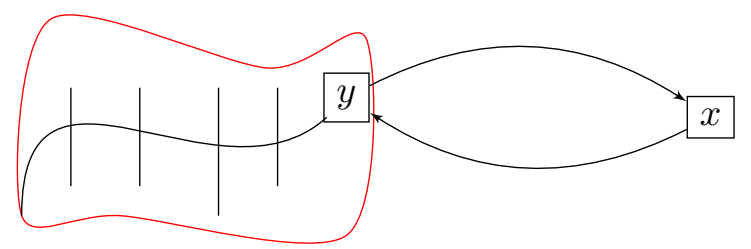

Figura 3.1: Distancia de um ponto ao Aubry.

Definição 23 (Potencial de Mañé). Dado o potencial $f$, chamamos o Potencial de Mañé à função dada por

$$
S_{f}(x, y):=\lim _{\epsilon \rightarrow 0} \sup _{n \geq 1} \sup _{\substack{z: d(x, z)<\epsilon \\ \sigma^{n}(z)=y}}\left\{S_{n}(f-m(f))(z)\right\} .
$$

O Potencial de Mañé $S_{f}(x, y)$ descreve em algum sentido "o caminho optimal " para ir de $x$ a y, seguindo a dinâmica, e também maximizando o "custo" dado por $f$.

Observação 6. Seja $\epsilon_{1}<\epsilon_{2}$ e $n \geq 1$ fixo, então claramente

$$
A:=\left\{z: d(x, z)<\epsilon_{1}, \sigma^{n}(z)=y\right\} \subseteq B:=\left\{z: d(x, z)<\epsilon_{2}, \sigma^{n}(z)=y\right\}
$$

dai

$$
\sup _{z \in A}\left\{S_{n}(f-m(f))(z)\right\} \leq \sup _{z \in B}\left\{S_{n}(f-m(f))(z)\right\},
$$

assim

$$
g\left(\epsilon_{1}\right):=\sup _{n \geq 1} \sup _{z \in A}\left\{S_{n}(f-m(f))(z)\right\} \leq \sup _{n \geq 1} \sup _{z \in B}\left\{S_{n}(f-m(f))(z)\right\}=: g\left(\epsilon_{2}\right),
$$

portanto a função

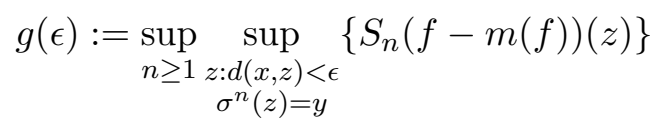

é monótona.

Proposição 5. Assuma $\left(\Sigma_{A}(\mathbb{N}), \sigma\right)$ é um shift primitivo. Seja $f: \Sigma_{A}(\mathbb{N}) \rightarrow \mathbb{R}$ com variação somável, tal que inf $\left.f\right|_{\bigcup_{i \in \mathbb{F}}}[i]>-\infty$, então $S_{f}(x, y)<\infty$.

Demonstração. Seja $\epsilon>0$ dado. Sejam $x$ e $y \in \Sigma_{A}(\mathbb{N})$, tomar um inteiro $k>K_{0}$ e um ponto

$$
z=\left(x_{0}, \ldots, x_{k-K_{0}-1}, w_{1}, \ldots, w_{K_{0}}, y_{0}, y_{1}, y_{2}, \ldots\right) \in \Sigma_{A}(\mathbb{N})
$$

verificando $d(x, z)<r^{k-K_{0}}$ e $\sigma^{k}(z)=y$; aqui $w_{1}, \ldots, w_{K_{0}} \in \mathbb{F}$.

Note que se $k<K_{0}$, neste caso temos que $S_{k}(f-m(f))(z) \leq k(\sup f-m(f)), \operatorname{logo}$

$$
S_{f}(x, y)<K_{0}(\sup f-m(f))<\infty .
$$

Note que podemos achar um ponto periódico

$$
z^{k}=\left(\overline{x_{0}, x_{1}, \ldots, x_{k-K_{0}-1}, l_{k-K_{0}}, l_{k-K_{0}+1}, \ldots, l_{k-1}}\right) \in \Sigma_{A}(\mathbb{N})
$$

de período $k$, com $l_{k-K_{0}}, l_{k-K_{0}+1}, \ldots, l_{k-1} \in \mathbb{F}$ tal que $d\left(z, z^{k}\right) \leq r^{k-K_{0}}$. 
Observe que

$$
\begin{aligned}
S_{k} f(z)-S_{k} f\left(z^{k}\right) & =\sum_{j=0}^{k-1} f\left(\sigma^{j}(z)\right)-\sum_{j=0}^{k-1} f\left(\sigma^{j}\left(z^{k}\right)\right) \\
& =\left[f(z)-f\left(z^{k}\right)\right]+\cdots+\left[f\left(\sigma^{k-1}(z)\right)-f\left(\sigma^{k-1}\left(z^{k}\right)\right)\right] \\
& =\left[f(z)-f\left(z^{k}\right)\right]+\cdots+\left[f\left(\sigma^{k-K_{0}-1}(z)\right)-f\left(\sigma^{k-K_{0}-1}\left(z^{k}\right)\right)\right] \\
& +\cdots+\left[f\left(\sigma^{k-1}(z)\right)-f\left(\sigma^{k-1}\left(z^{k}\right)\right)\right] \\
& \leq \operatorname{Var}_{k-K_{0}}(f)+\cdots+\operatorname{Var}_{1}(f)+K_{0}\left(\sup f-\left.\inf f\right|_{\cup_{i \in \mathbb{F}}[i]}\right) .
\end{aligned}
$$

Como $z^{k}$ é periódico temos que $\mu:=\frac{1}{k} \sum_{j=0}^{k-1} \delta_{\sigma^{j}}\left(z^{k}\right)$ é uma medida $\sigma$-invariante, assim obtemos

$$
S_{k} f\left(z^{k}\right) \leq k m(f),
$$

portanto

$$
\begin{aligned}
S_{k}(f-m(f))(z) & \leq \operatorname{Var}_{k-K_{0}}(f)+\cdots+\operatorname{Var}_{1}(f)+K_{0}\left(\sup f-\left.\inf f\right|_{\cup_{i \in \mathbb{E}}[i]}\right) \\
& \leq \operatorname{Var}(f)+K_{0}\left(\sup f-\left.\inf f\right|_{\cup_{i \in \mathbb{E}}[i]}\right) .
\end{aligned}
$$

Portanto obtemos

$$
\sup _{k \geq 1, \bar{z} \in \Sigma_{A}(\mathbb{N})}\left\{S_{k}(f-m(f))(\bar{z}) \mid k \geq 1, d(x, \bar{z})<\epsilon, \sigma^{k}(\bar{z})=y\right\} \leq L,
$$

donde $L$ é uma constante real dada por $L:=\operatorname{Var}(f)+K_{0}\left(\sup f-\left.\inf f\right|_{\cup_{i \in \mathbb{F}}[i]}\right)$.

Em consequência tomando o limite quando $\epsilon \rightarrow 0$ concluímos finalmente que $S_{f}(x, y)<\infty$.

Observação 7. Observe que a condição inf $\left.f\right|_{\bigcup_{i \in \mathbb{F}}}[i]>-\infty$ é verificada quando $\mathbb{F}$ é finita. Com efeito, escolha um ponto $x^{i} \in[i]$ para cada $i \in \mathbb{F}$, se $x \in[j], j \in \mathbb{F}$, então $f(x)>f\left(x^{j}\right)-\operatorname{Var}_{1}(f)$ claramente implica inf $\left.f\right|_{\bigcup_{i \in \mathbb{F}}}[i]>\min _{i \in \mathbb{F}} f\left(x^{i}\right)-\operatorname{Var}_{1}(f)>-\infty$.

Definição 24 (Barreira de Peierls). A Barreira de Peierls entre $x$ e y é definida por

$$
h_{f}(x, y):=\lim _{\epsilon \rightarrow 0} \limsup _{n \rightarrow \infty} \sup _{\substack{z: d(x, z)<\epsilon \\ \sigma^{n}(z)=y}}\left\{S_{n}(f-m(f))(z)\right\} .
$$

Observe que

$$
h_{f}(x, y) \leq S_{f}(x, y)
$$

Ambas definições, o Potencial de Mañé e a Barreira de Peierls são bastante similares, exceto que no primeiro caso consideramos o supremo, e no outro caso consideramos o lim sup.

Note que de (3.23) sabemos que

$$
S_{k}(f-m(f)) \leq\left(k-K_{0}\right) \operatorname{Var}_{1}(f)+K_{0}\left(\sup f-\left.\inf f\right|_{\cup_{i \in \mathbb{F}}[i]}\right)
$$

assim tomando o supremo sobre $z$ e limsup sobre $k$, obtemos

$$
\limsup _{k} \sup _{z \in \Sigma_{A}(\mathbb{N})}\left\{S_{k}(f-m(f))(z) \mid d(x, z)<\epsilon, \sigma^{k}(z)=y\right\} \leq L,
$$

logo tomando o limite quando $\epsilon \rightarrow 0$ concluímos que $h_{f}(x, y)<\infty$.

$\mathrm{Na}$ seguinte proposição mostramos algumas propriedades acerca da Barreira de Peierls e o Potencial de Mañé. Vamos usar as idéias de [BLL13, Gar09, CLT01, GL08, GLT09, Gar06, CLO13] adaptado ao caso de um shift enumerável e finitamente primitivo.

Proposição 6. Seja $\left(\Sigma_{A}(\mathbb{N}), \sigma\right)$ um shift finitamente primitivo. Seja $f: \Sigma_{A}(\mathbb{N}) \rightarrow \mathbb{R}$ com variação somável e $P(f)<\infty$, e $V$ uma sub-ação calibrada limitada com variação somável, então: 
(a) $S_{f}(x, x) \leq 0$, para cada $x \in \Sigma_{A}(\mathbb{N})$.

(b) Para quaisquer $x, y, z \in \Sigma_{A}(\mathbb{N})$,

$$
\begin{aligned}
& S_{f}(x, y)+S_{f}(y, z) \leq S_{f}(x, z) . \\
& h_{f}(x, y)+h_{f}(y, z) \leq h_{f}(x, z) .
\end{aligned}
$$

(c) Para qualquer $x \in \Omega(f, \sigma), S_{f}(x, \cdot)>-\infty$.

(d) Para qualquer $x, y \rightarrow S_{f}(x, y)$ tem variação somável.

(e) $h_{f}(x, \cdot)=S_{f}(x, \cdot)$ quando $x \in \Omega(f, \sigma)$.

(f) Assumindo que $\Omega(f, \sigma)=\operatorname{supp}(\mu)$ temos que, para $x \in \Omega(f, \sigma), S_{f}(x, \cdot)$ é uma sub-ação calibrada.

(g) $\Omega(f, \sigma)=\left\{x \in \Sigma_{A}(\mathbb{N}): S_{f}(x, x)=0\right\}$.

(h) $\operatorname{supp}(\mu) \subset \Omega(f, \sigma)=\left\{x \in \Sigma_{A}(\mathbb{N}): S_{f}(x, x)=0\right\}$, onde $\mu \in \mathcal{M}_{\max }(f)$.

(i) Se $\mathcal{M}_{\max }(f)=\{\mu\}$ e $a, b \in \Omega(f, \sigma)=\operatorname{supp}(\mu)$ então $S_{f}(a, b)+S_{f}(b, a)=0$.

(j) Se $S_{f}\left(x_{0}, \sigma^{n_{k}}\left(x_{0}\right)\right)=\sum_{j=0}^{n_{k}-1} f\left(\sigma^{j}\left(x_{0}\right)\right), \lim _{k \rightarrow \infty} \sigma^{n_{k}}\left(x_{0}\right)=b e$ $\lim _{k \rightarrow \infty} n_{k}=+\infty$ então

$$
\lim _{k \rightarrow \infty} S_{f}\left(x_{0}, \sigma^{n_{k}}\left(x_{0}\right)\right)=S_{f}\left(x_{0}, b\right) .
$$

Demonstração.

\section{Notação 1.}

$$
\begin{gathered}
S_{f}^{\epsilon}(x, y):=\sup _{n \geq 1, z \in \Sigma_{A}(\mathbb{N})}\left\{S_{n}(f-m(f))(z) \mid n \geq 1, d(x, z)<\epsilon, \sigma^{n}(z)=y\right\} . \\
S_{f}^{\epsilon, l}(x, y):=\sup _{n>l} \sup _{\substack{z: d(x, z)<\epsilon \\
\sigma^{n}(z)=y}}\left\{S_{n}(f-m(f))(z)\right\} .
\end{gathered}
$$

Afirmação 3. $S_{f}(x, y) \leq S_{f}^{\epsilon}(x, y)$

Demonstração. Fixado $\epsilon>0$, tomar $\epsilon^{\prime} \in(0, \epsilon)$, considere um ponto $z \in \Sigma_{A}(\mathbb{N})$ tal que $d(x, z)<\epsilon^{\prime}$, $\sigma^{k}(z)=y \mathrm{e}$

$$
S_{f}^{\epsilon^{\prime}}(x, y)-\epsilon^{\prime}<\sum_{j=0}^{k-1}(f-m(f))\left(\sigma^{j}(z)\right)
$$

assim

$$
S_{f}^{\epsilon}(x, y) \geq \sum_{j=0}^{k-1}(f-m(f))\left(\sigma^{j}(z)\right)>S_{f}^{\epsilon^{\prime}}(x, y)-\epsilon^{\prime},
$$

logo tomando o limite quando $\epsilon^{\prime} \rightarrow 0$ temos $S_{f}(x, y) \leq S_{f}^{\epsilon}(x, y)$.

(a). Seja $\rho>0$ e tome $\epsilon \in(0, \rho)$. Sejam $x, y \in \Sigma_{A}(\mathbb{N})$ dados. Seja $z \in \Sigma_{A}(\mathbb{N})$ e $n \in \mathbb{N}$ tais que $d(x, z)<\epsilon$ e $\sigma^{n}(z)=y$. Pela continuidade de $V$ temos que

$$
d(x, z)<\epsilon \Rightarrow|V(x)-V(z)|<\rho, \forall x, z \in \Sigma_{A}(\mathbb{N}) .
$$


Então

$$
V(x)-V(y)=V(x)-V\left(\sigma^{n}(z)\right)<V(z)+\rho-V\left(\sigma^{n}(z)\right)
$$

assim

$$
V(x)-V(y)-\rho<V(z)-V\left(\sigma^{n}(z)\right) \leq-S_{n}(f-m(f))(z),
$$

portanto

$$
S_{n}(f-m(f))(z) \leq V(y)-V(x)+\rho,
$$

logo, tomando o supremo sobre todos os $z$ tales que $d(x, z)<\epsilon$ e $\sigma^{n}(z)=y$ e o supremo em $n$ obtemos

$$
\sup _{n} \sup _{z} S_{n}(f-m(f))(z) \leq V(y)-V(x)+\rho,
$$

fazendo $\rho \rightarrow 0$ segue que

$$
S_{f}(x, y) \leq V(y)-V(x) .
$$

Em particular obtemos que $S_{f}(x, x) \leq 0$.

(b). Tomar $x, y, z \in \Sigma_{A}(\mathbb{N})$. Fixar uma constante $\eta>0$ e um inteiro positivo $k$. Considere os inteiros $l_{1}, l_{2} \geq 0$. Existe um ponto

$$
b=\left(b_{0}, b_{1}, \ldots\right) \in \Sigma_{A}(\mathbb{N})
$$

e um inteiro $n>l_{1}$ tal que

$$
d(x, b) \leq r^{k}, \quad \sigma^{n}(b)=y
$$

e

$$
S^{r^{k}, l_{1}}(x, y)-\eta<S_{n}(f-m(f))(b) .
$$

Existem também um ponto $\bar{b}=\left(\bar{b}_{0}, \bar{b}_{1}, \ldots\right) \in \Sigma_{A}(\mathbb{N})$ e um inteiro $m>l_{2}$ tal que

$$
d(y, \bar{b}) \leq r^{k}, \quad \sigma^{m}(\bar{b})=z
$$

e

$$
S^{r^{k}, l_{2}}(y, z)-\eta<S_{m}(f-m(f))(\bar{b}) .
$$

Defina

$$
\bar{c}=\left(b_{0}, b_{1}, \ldots, b_{n-1}, \bar{b}_{0}, \bar{b}_{1}, \ldots\right) \in \Sigma_{A}(\mathbb{N}) .
$$

Note que por construção

$$
\left(\bar{b}_{0}, \bar{b}_{1}, \ldots, \bar{b}_{k}\right)=\left(y_{0}, y_{1}, \ldots, y_{k}\right)=\left(b_{n}, \ldots, b_{n+k}\right) .
$$

Verificamos

$$
d(\bar{c}, x) \leq d(\bar{c}, b)+d(b, x) \leq r^{n+k}+r^{k}<2 r^{k}
$$

e

$$
\sigma^{n+m}(\bar{c})=z .
$$

Portanto temos

$$
\begin{aligned}
S_{f}^{2 r^{k}, l_{1}+l_{2}}(x, z) & \geq S_{n+m}(f-m(f))(\bar{c}) \\
& =S_{n}(f-m(f))(\bar{c})+(f-m(f))\left(\sigma^{n}(\bar{c})\right) \\
& +\cdots+(f-m(f))\left(\sigma^{n+m-1}(\bar{c})\right) \\
& =S_{n}(f-m(f))(\bar{c})+S_{m}(f-m(f))(\bar{b}) .
\end{aligned}
$$


Observe que

$$
\begin{aligned}
S_{n}(f-m(f))(\bar{c}) & =S_{n}(f-m(f))(b)-S_{n}(f)(b)+S_{n}(f)(\bar{c}) \\
& >S_{f}^{r^{k}, l_{1}}(x, y)-\eta-\left(\operatorname{Var}_{n+k}(f)+\cdots+\operatorname{Var}_{k+1}(f)\right)
\end{aligned}
$$

por conseguinte

$$
S_{f}^{2 r^{k}, l_{1}+l_{2}}(x, z)>S_{f}^{r^{k}, l_{2}}(y, z)+S_{f}^{r^{k}, l_{1}}(x, y)-n \operatorname{Var}_{k+l}(f)-2 \eta .
$$

Fazendo $l_{1}=l_{2}=0$ e tomando o limite quando $k$ vai para infinito e considerando $\eta>0$ arbitrariamente pequeno, obtemos (3.26). Observe que da equação (3.31) segue também que

$$
\sup _{l_{1}, l_{2} \geq 0} S_{f}^{2 r^{k}, l_{1}+l_{2}}(x, z)>\sup _{l_{2}} S_{f}^{r^{k}, l_{2}}(y, z)+\sup _{l_{1}} S_{f}^{r^{k}, l_{1}}(x, y)-n \operatorname{Var}_{k+1} S_{n}(f)-2 \eta
$$

e então repetindo o processo anterior tem-se (3.27).

(c). Fixados $x \in \Omega(f, \sigma), y \in \Sigma_{A}(\mathbb{N})$ e $2 \eta \in(0, \delta)$. Existe um ponto $z=\left(z_{0}, z_{1}, \ldots\right) \in \Sigma_{A}(\mathbb{N})$ e um inteiro $n>0$ tal que

$$
d(x, z) \leq \delta, \sigma^{n}(z)=y
$$

e

$$
S_{f}^{r}(x, y)-\eta<S_{n}(f-m(f))(z) .
$$

Como $x$ é um ponto do Aubry, podemos achar $\bar{z}=\left(\bar{z}_{0}, \bar{z}_{1}, \ldots\right) \in \Sigma_{A}(\mathbb{N})$ e $m>0$ tal que

$$
d(x, \bar{z})<\eta, \quad \sigma^{m}(\bar{z})=x
$$

e

$$
\left|S_{m}(f-m(f))(\bar{z})\right|<\eta .
$$

Aqui consideramos $m>0$ suficientemente grande tal que $r^{m}<\eta$. Considere o ponto $w:=$ $\left(\bar{z}_{0}, \bar{z}_{1}, \ldots, \bar{z}_{m-1}, z_{0}, \ldots, z_{n-1}, \ldots\right) \in \Sigma_{A}(\mathbb{N})$, assim

$$
d(w, x) \leq d(w, \bar{z})+d(\bar{z}, x) \leq r^{m}+\eta<2 \eta
$$

e $\sigma^{n+m}(w)=\sigma^{n}(z)=y$. Portanto, temos

$$
\begin{aligned}
S_{f}^{2 \eta, 0}(x, y) & \geq S_{n+m}(f-m(f))(w) \\
& =S_{m}(f-m(f))(w)+(f-m(f))\left(\sigma^{m}(w)\right)+\cdots+(f-m(f))\left(\sigma^{m+n-1}(w)\right) \\
& =S_{m}(f-m(f))(w)+S_{n}(f-m(f))(z) .
\end{aligned}
$$

E como

$$
\begin{aligned}
S_{m}(f-m(f))(w) & =S_{m}(f-m(f))(\bar{z})-S_{m} f(\bar{z})+S_{m} f(w) \\
& >-\eta-\left(\operatorname{Var}_{m}(f)+\cdots+\operatorname{Var}_{1}(f)\right),
\end{aligned}
$$

obtemos

$$
\begin{aligned}
S_{f}^{2 \eta, 0}(x, y) & >-\eta-\left(\operatorname{Var}_{m}(f)+\cdots+\operatorname{Var}_{1}(f)\right)+S_{n}(f-m(f))(z) \\
& >-2 \eta-\operatorname{Var}(f)+S_{f}^{r}(x, y) .
\end{aligned}
$$

Deste modo quando $\eta$ vai para 0 , concluímos que

$$
S_{f}(x, y)>S_{f}^{r}(x, y)-\operatorname{Var}(f)>-\infty
$$


(d). Fixados $\epsilon>0$ e $x, \bar{y} \in \Sigma_{A}(\mathbb{N})$. Tome $y \in \Sigma_{A}(\mathbb{N})$ tal que $d(y, \bar{y}) \leq r^{n+k}$, com $n, k \geq 1 \mathrm{e}$ $\rho \in(0, \epsilon)$. Considere $\bar{z}:=\left(z_{0}, z_{1}, \ldots, z_{\bar{k}-1}, \bar{y}_{0}, \bar{y}_{1}, \ldots\right)$ tal que $d(x, \bar{z})<\epsilon, \sigma^{\bar{k}}(\bar{z})=\bar{y}$ e

$$
S_{f}^{\epsilon}(x, \bar{y})-\rho<\sum_{j=0}^{\bar{k}-1}(f-m(f))\left(\sigma^{j}(\bar{z})\right) .
$$

E considere $z:=\left(z_{0}, z_{1}, \ldots, z_{\bar{k}-1}, y_{0}, y_{1}, \ldots\right)$ cumprindo $d(x, z)<\epsilon$ e $\sigma^{\bar{k}}(z)=y$.

Observe que

$$
\begin{aligned}
& \sum_{j=0}^{\bar{k}-1}(f-m(f))\left(\sigma^{j}(\bar{z})\right)-\sum_{j=0}^{\bar{k}-1}(f-m(f))\left(\sigma^{j}(z)\right) \\
& =[f(\bar{z})-f(z)]+\cdots+\left[f\left(\sigma^{\bar{k}-1}(\bar{z})\right)-f\left(\sigma^{\bar{k}-1}(z)\right)\right] \\
& \leq \operatorname{Var}_{k+\bar{k}}(f)+\operatorname{Var}_{k+\bar{k}-1}(f)+\cdots+\operatorname{Var}_{k+1}(f) \\
& \leq \bar{k} \operatorname{Var}_{k+1}(f) .
\end{aligned}
$$

Deste modo

$$
\begin{aligned}
S_{f}(x, \bar{y}) & \leq S_{f}^{\epsilon}(x, \bar{y}) \quad \text { (Observação 3) } \\
& <\sum_{j=0}^{\bar{k}-1}(f-m(f))\left(\sigma^{j}(\bar{z})\right)+\rho \\
& \leq \bar{k} \operatorname{Var}_{k+1}(f)+\sum_{j=0}^{\bar{k}-1}(f-m(f))\left(\sigma^{j}(z)\right)+\rho \\
& \leq S_{f}^{\epsilon}(x, y)+\bar{k} \operatorname{Var}_{k+1}(f)+\rho .
\end{aligned}
$$

Fazendo $\epsilon \rightarrow 0$ concluímos que

$$
S_{f}(x, \bar{y})-S_{f}(x, y) \leq \bar{k} \operatorname{Var}_{k+1}(f),
$$

portanto

$$
\operatorname{Var}\left(S_{f}(x, \cdot)\right)=\sum_{k=1}^{\infty} \operatorname{Var}_{k}\left(S_{f}(x, \cdot)\right) \leq \bar{k} \sum_{k=1}^{\infty} \operatorname{Var}_{k}(f)<\infty .
$$

Logo $S_{f}(x, \cdot)$ tem variação somável.

(e). Dado $\epsilon>0$ e considere uma sequência de inteiros positivos

$$
0<k_{1}<k_{2}<\ldots<k_{m}<\ldots \text { tal que } r^{k_{m}}<\frac{\epsilon}{2^{m}}
$$

Podemos achar um ponto $y^{1}=\left(y_{0}^{1}, y_{1}^{1}, \ldots\right) \in \Sigma_{A}(\mathbb{N})$ e um inteiro $n_{1}>0$ satisfazendo $d\left(x, y^{1}\right)<r^{k_{1}}$, $\sigma^{n_{1}}\left(y^{1}\right)=y \mathrm{e}$

$$
S_{f}^{r^{k_{1}}, 0}(x, y)-\epsilon / 2<S_{n_{1}}(f-m(f))\left(y^{1}\right) .
$$

Dado $x \in \Omega(f, \sigma)$, para cada índice $m>1$, podemos determinar $y^{m}=\left(y_{0}^{m}, y_{1}^{m}, \ldots\right) \in \Sigma_{A}(\mathbb{N})$ e um inteiro $n_{m}$ satisfazendo

$$
d\left(x, y^{m}\right) \leq r^{k_{m}}<\frac{\epsilon}{2^{m}}, \sigma^{n_{m}}\left(y^{m}\right)=x
$$

e

$$
S_{n_{m}}(f-m(f))\left(y^{m}\right)>-\frac{\epsilon}{2^{m}}
$$


Agora, fixando um inteiro não negativo $l$, definimos

$$
\bar{y}:=\left(y_{0}^{l+1}, y_{1}^{l+1}, \ldots, y_{n_{l}}^{l+1}, y_{0}^{l}, y_{1}^{l}, \ldots, y_{n_{l}-1}^{l}, \ldots, y_{0}^{2}, y_{1}^{2}, \ldots, y_{n_{2}-1}^{2}, y_{0}^{1}, y_{1}^{1}, \ldots\right) \in \Sigma_{A}(\mathbb{N})
$$

e

$$
n:=n_{l+1}+n_{l}+\ldots+n_{2}+n_{1}>l .
$$

Observe que, para todo $m>1$, temos

$$
\left(y_{n_{m}}^{m}, y_{n_{m}+1}^{m}, \ldots, y_{n_{m}+k_{m-1}}^{m}\right)=\left(x_{0}, x_{1}, \ldots, x_{k_{m-1}}\right)=\left(y_{0}^{m-1}, y_{1}^{m-1}, \ldots, y_{k_{m-1}}^{m-1}\right) .
$$

Em particular, segue que

$$
d(x, \bar{y}) \leq d\left(x, y^{l+1}\right)+d\left(y^{l+1}, \bar{y}\right) \leq r^{k_{l+1}}+r^{n_{l+1}+k_{l}}<2 r^{k_{l}}<\frac{\epsilon}{2^{l-1}} .
$$

além disso,

$$
\sigma^{n}(\bar{y})=\sigma^{n_{1}}\left(y^{1}\right)=y \text {. }
$$

Então para $l>1$, resulta

$$
\begin{aligned}
S_{f}^{\frac{\epsilon}{2}, l}(x, y) & \geq S_{n}(f-m(f))(\bar{y}) \\
& =S_{n_{l+1}}(f-m(f))(\bar{y})+S_{n_{l}}(f-m(f))\left(\sigma^{n_{l+1}}(\bar{y})\right)+\cdots \\
& +S_{n_{2}}(f-m(f))\left(\sigma^{n_{l+1}+\cdots+n_{3}}(\bar{y})\right)+S_{n_{1}}(f-m(f))\left(y^{1}\right) .
\end{aligned}
$$

Também temos

$$
\begin{aligned}
& S_{n_{2}}(f-m(f))\left(\sigma^{n_{l+1}+\cdots+n_{3}}(\bar{y})\right) \\
& =S_{n_{2}}(f-m(f))\left(y^{2}\right)-S_{n_{2}} f\left(y^{2}\right)+S_{n_{2}} f\left(\sigma^{n_{l+1}+\cdots+n_{3}}(\bar{y})\right) \\
& >-\frac{\epsilon}{4}-\left(\operatorname{Var}_{n_{2}+k_{1}+1}(f)+\cdots+\operatorname{Var}_{k_{1}+2}(f)\right) \\
& >-\frac{\epsilon}{4}-n_{2} \operatorname{Var}_{k_{1}+2}(f) .
\end{aligned}
$$

similarmente

$$
\begin{aligned}
& S_{n_{l+1}}(f-m(f))(\bar{y}) \\
& =S_{n_{l+1}}(f-m(f))\left(y^{l+1}\right)-S_{n_{l+1}} f\left(y^{l+1}\right)+S_{n_{l+1}} f(\bar{y}) \\
& >-\frac{\epsilon}{2^{l+1}}-\left(\operatorname{Var}_{n_{l}+k_{l}+1}(f)+\cdots+\operatorname{Var}_{k_{l}+2}(f)\right) \\
& >-\frac{\epsilon}{2^{l+1}}-n_{l+1} \operatorname{Var}_{k_{l}+2}(f) .
\end{aligned}
$$

Por conseguinte

$$
\begin{aligned}
S_{f}^{\frac{\epsilon}{2}, l}(x, y) & >S_{f}^{r^{k_{1}}, 0}(x, y)-\left(\frac{\epsilon}{2}+\frac{\epsilon}{4}+\cdots+\frac{\epsilon}{2^{l+1}}\right)-\left(n_{2}+\cdots+n_{l+1}\right) \operatorname{Var}_{k_{1}+2}(f) \\
& >S_{f}^{r^{k_{1}, 0}}(x, y)-\epsilon-\left(n_{2}+\cdots+n_{l+1}\right) \operatorname{Var}_{k_{1}+2}(f)
\end{aligned}
$$

Fazendo $k_{1} \rightarrow \infty$ obtemos

$$
S_{f}^{\frac{\epsilon}{2}, l}(x, y)>S_{f}(x, y)-\epsilon
$$

Tomando o ínfimo em $l>1$ e considerando $\epsilon>0$ arbitrariamente pequeno, segue que

$$
h_{f}(x, y) \geq S_{f}(x, y) .
$$


(f). Seja $x \in \Omega(f, \sigma)=\operatorname{supp}(\mu)$, e $\bar{x} \in \Sigma_{A}(\mathbb{N})$ tais que $\sigma(\bar{x})=\bar{x}$. Pelo item $(b)$ sabemos que

$$
S_{f}(x, \sigma(\bar{x}))-S_{f}(x, \bar{x}) \geq S_{f}(\bar{x}, \sigma(\bar{x})) \geq(f-m(f))(\bar{x}) .
$$

Por conseguinte $S_{f}(x, \cdot)$ é uma sub-ação. Assim resta provar que

$$
S_{f}(x, \bar{x}) \leq S_{f}(x, \bar{y})+f(\bar{y})-m(f)
$$

para algum $\bar{y} \in \Sigma_{A}(\mathbb{N})$ com $\sigma(\bar{y})=\bar{x}$. Com efeito, dado um inteiro $k \geq 1$, existe $z^{k} \in \Sigma_{A}(\mathbb{N})$ e $n_{k} \in \mathbb{N}$ tal que

$$
d\left(z^{k}, x\right) \leq r^{k}<\frac{\epsilon}{2}, \sigma^{n_{k}}\left(z^{k}\right)=\bar{x}
$$

$\mathrm{e}$

$$
S_{f}^{r^{k}, 0}(x, \bar{x})-\frac{1}{k}<S_{n_{k}}(f-m(f))\left(z^{k}\right) .
$$

Como $x \in \Omega(f, \sigma)=\operatorname{supp}(\mu), \lim _{k \rightarrow \infty} z^{k}=x$ e $\operatorname{supp}(\mu)$ está contido num shift compacto, (veja [JMU06, RJ14, RG10]) digamos $\Sigma_{A}(J)$, então $\left(z^{k}\right)$ é da forma:

$z^{k}:=\left(x_{0}, \ldots, x_{l}, z_{l+1}^{k}, z_{l+2}^{k}, \ldots\right)$; onde cada $x_{i} \in\{0, \ldots, J\}$. Em seguida construímos uma subsequência $\left(z^{k_{j}}\right)$ de $\left(z^{k}\right)$ da forma: $z^{k_{j}}:=\left(x_{0}, \ldots, x_{l}, b_{j_{1}}, b_{j_{2}}, \ldots\right)$, onde cada $b_{j_{i}} \in\{0, \ldots, J\}$. Desta forma existe $y^{\prime}$ ponto limite da subsequência $\left(z^{k_{j}}\right)_{j>0}$, isto é para $j$ suficientemente grande segue que

$$
d\left(z^{k_{j}}, y^{\prime}\right)<\frac{\epsilon}{2}
$$

em consequência

$$
d\left(x, y^{\prime}\right) \leq d\left(y^{\prime}, z^{k_{j}}\right)+d\left(z^{k_{j}}, x\right)<\frac{\epsilon}{2}+\frac{\epsilon}{2}=\epsilon .
$$

Observe também que

$$
\lim _{j \rightarrow \infty} \sigma^{n_{k_{j}}-1}\left(z^{k_{j}}\right):=\bar{y}
$$

e

$$
\bar{x}=\lim _{j \rightarrow \infty} \sigma^{n_{k_{j}}}\left(z^{k_{j}}\right)=\sigma(\bar{y}) .
$$

Agora para $\epsilon>0$, e $j$ suficientemente grande, obtemos

$$
\begin{aligned}
S_{f}^{\epsilon, 0}(x, \bar{y})+f\left(\sigma^{n_{k_{j}}-1}\left(z^{k_{j}}\right)\right)-m(f) & \geq S_{n_{k_{j}}-1}(f-m(f))\left(z^{k_{j}}\right)+f\left(\sigma^{n_{k_{j}}-1}\left(z^{k_{j}}\right)\right)-m(f) \\
& =S_{n_{k_{j}}}(f-m(f))\left(z^{k_{j}}\right)-f\left(\sigma^{n_{k_{j}}-1}\left(z^{k_{j}}\right)\right)+f\left(\sigma^{n_{k_{j}}-1}\left(z^{k_{j}}\right)\right) \\
& >S_{f}^{r^{k_{j}, 0}}(x, \bar{x})-\frac{1}{k_{j}}
\end{aligned}
$$

assim fazendo o limite quando $j$ vai para infinito e considerando $\epsilon>0$ arbitrariamente pequeno, temos

$$
S_{f}(x, \bar{y})+f(\bar{y})-m(f) \geq S_{f}(x, \bar{x}) .
$$

(g). Suponha $x \in \Omega(f, \sigma)$ então para $\epsilon>0$ existe $z \in \Sigma_{A}(\mathbb{N})$ e $k \in \mathbb{N}$ tal que $d(x, z)<\epsilon, \sigma^{k}(z)=x$ e $\left|S_{k}(f-m(f))(z)\right|<\epsilon$. Assim

$$
\sup _{n} \sup _{z}\left|\sum_{j=0}^{n-1} f\left(\sigma^{j}(z)-m(f)\right)\right|<\epsilon,
$$

fazendo $\epsilon \rightarrow 0$ segue que $S_{f}(x, x) \geq 0$ e como $S_{f}(x, x) \leq 0$ concluímos que $S_{f}(x, x)=0$.

Suponha agora que $x \in \Sigma_{A}(\mathbb{N}) \operatorname{com} S_{f}(x, x)=0$ e $x \notin \Omega(f, \sigma)$, então $\exists \epsilon_{0}>0$ tal que $\forall z \in \Sigma_{A}(\mathbb{N})$ 
e $k \in \mathbb{N}$, se $d(x, z)<\epsilon, \sigma^{k}(z)=x$

$$
\Rightarrow \sup _{n} \sup _{z} \sum_{j=0}^{k-1}\left(f\left(\sigma^{j}(z)-m(f)\right)\right) \geq \epsilon_{0} .
$$

isso contradiz que $S_{f}(x, x)=0$.

Lema 11. Seja $\mu$ uma medida invariante ergódica. Seja $x$ um ponto qualquer no $\operatorname{supp}(\mu)$. Então $x$ retorna infinitas vezes perto dele mesmo.

Demonstração. Seja $\epsilon>0$, e considere a bola $B(x, \epsilon)$. Como $B(x, \epsilon)$ é aberta e fechada então $1_{B(x, \epsilon)}$ é contínua. Assim

$$
\lim _{n \rightarrow \infty} \frac{1}{n} \sum_{j=0}^{n-1} 1_{B(x, \epsilon)}\left(\sigma^{j}(x)\right)=\mu(B(x, \epsilon))>0 .
$$

Portanto há infinitos $n$ tais que $1_{B(x, \epsilon)}\left(\sigma^{n}(x)\right)=1$.

(h). Seja $V$ uma sub-ação calibrada limitada com variação somável e $x \in \operatorname{supp}(\mu)$, assim

$$
V(\sigma(x)) \geq f(x)-m(f)+V(x),
$$

qual pode ser escrito como

$$
f(x)=m(f)+V \circ \sigma(x)-V(x)+g(x),
$$

donde $g$ é uma função com variação somável, limitada superiormente e não positiva. Logo

$$
S_{n}(f-m(f))(x)=S_{n}(g)(x)+V \circ \sigma^{n}(x)-V(x) .
$$

Como $\lim _{n \rightarrow \infty} \frac{1}{n} S_{n}(f-m(f))(x)=0$ ( $\mu$ é $f$-maximizante), então

$\lim _{n \rightarrow \infty} \frac{1}{n}\left(S_{n} g(x)+V \circ \sigma^{n}(x)-V(x)\right)=0$, portanto $\int g d \mu=0$, em consequência $\left.g\right|_{\operatorname{supp} \mu} \equiv 0$. Isto implica que

$$
f(x)=m(f)+V \circ \sigma(x)-V(x) .
$$

Note que $x$ regressa infinitas vezes arbitrariamente perto dela mesma (veja Lema 11). Deste modo, para $\epsilon=r^{N}$ dado, existem infinitos $n_{i}$ tal que

$$
x=x_{0} x_{1} \ldots x_{n_{i}-1} x_{0} x_{1} \ldots x_{N-1} \ldots
$$

isto significa que a palavra $x_{0} \ldots x_{N-1}$ aparece infinitas vezes em $x=x_{0} x_{1} \ldots$ Então para tal $n_{i}$, a palavra

$$
z=x_{0} x_{1} \ldots x_{n_{i}-1} x
$$

coincide com $x$ em pelo menos $n_{i}+N$ dígitos. Como $g$ tem variação somável temos que

$$
\begin{aligned}
\left|S_{n_{i}}(g)(z)-S_{n_{i}}(g)(x)\right| & \leq \operatorname{Var}_{N+n_{i}}(g)+\cdots+\operatorname{Var}_{N+1}(g) \\
& \leq n_{i} \operatorname{Var}_{N+1}(g) .
\end{aligned}
$$

Agora usando que $V$ tem variação somável e $g \circ \sigma^{k}(x)=0(x \in \operatorname{supp}(\mu))$ para cada $k=0,1,2, \ldots$, obtemos

$$
\sigma^{n_{i}}(z)=x, \quad d(z, x) \leq r^{n_{i}+N}
$$


e

$$
\begin{aligned}
\left|S_{n_{i}}(f-m(f))(z)\right| & \leq\left|S_{n_{i}}(g)(z)-S_{n_{i}}(g)(x)\right|+\left|V \circ \sigma^{n_{i}}(z)-V(z)\right| \\
& \leq n_{i} \operatorname{Var}_{N+1}(g)+\operatorname{Var}_{n_{i}+N}(V),
\end{aligned}
$$

fazendo $N \rightarrow \infty$ obtemos que $S_{n_{i}}(f-m(f))(z)=0, \operatorname{assim} S_{f}(x, x) \geq 0$. E como $S_{f}(x, x) \leq 0$ obtemos que $x$ pertence a $\Omega(f, \sigma)$.

(i). Sem perda de generalidade, assumimos $m(f)=0$.

Seja $a, b \in \operatorname{supp}(\mu)=\Omega(f, \sigma)$. Como $a \in \operatorname{supp}(\mu)$ então existe $\alpha_{k} \rightarrow a, m_{k} \uparrow \infty, \sigma^{m_{k}}\left(\alpha_{k}\right)=a$ tal que $\lim _{k \rightarrow \infty} \sum_{j=0}^{m_{k}-1} f\left(\sigma^{j}\left(\alpha_{k}\right)\right)=0$.

Como $b \in \operatorname{supp}(\mu)$ existe $n_{k} \leq m_{k}$ tal que $\lim _{k \rightarrow \infty} \sigma^{n_{k}}\left(\alpha_{k}\right)=b . \operatorname{Assim} d\left(\sigma^{n_{k}}\left(\alpha_{k}\right), b\right) \leq r^{l}$, para algum $l \geq 1$. Seja $\tau_{k}$ o ramo inverso de $\sigma^{n_{k}}$ tal que $\tau_{k}\left(\sigma^{n_{k}}\left(\alpha_{k}\right)\right)=\alpha_{k}$. Seja $b_{k}:=\tau_{k}(b)$. Então $\sigma^{n_{k}}\left(b_{k}\right)=b \mathrm{e}$

$$
\begin{aligned}
d\left(b_{k}, a\right) & \leq d\left(b_{k}, \alpha_{k}\right)+d\left(\alpha_{k}, a\right) \\
& \leq r^{n_{k}} d\left(b, \sigma^{n_{k}}\left(\alpha_{k}\right)\right)+d\left(\alpha_{k}, a\right) \leq d\left(b, \sigma^{n_{k}}\left(\alpha_{k}\right)\right)+d\left(\alpha_{k}, a\right) \rightarrow 0 \quad(k \rightarrow \infty) .
\end{aligned}
$$

Note que

$$
d\left(b_{k}, \alpha_{k}\right) \leq r^{n_{k}} d\left(b, \sigma^{n_{k}}\left(\alpha_{k}\right)\right) \leq r^{n_{k}} r^{l}=r^{n_{k}+l} .
$$

Desta maneira

$$
\begin{aligned}
\left|\sum_{j=0}^{n_{k}-1} f\left(\sigma^{j}\left(b_{k}\right)\right)-\sum_{j=0}^{n_{k}-1} f\left(\sigma^{j}\left(\alpha_{k}\right)\right)\right| & \leq \operatorname{Var}_{n_{k}+l}(f)+\cdots+\operatorname{Var}_{l+1}(f) \\
& \leq n_{k} \operatorname{Var}_{l+1}(f) .
\end{aligned}
$$

Logo

$$
\begin{aligned}
S_{f}(a, b) & \geq \limsup _{k \rightarrow \infty} \sum_{j=0}^{n_{k}-1} f\left(\sigma^{j}\left(b_{k}\right)\right) \\
& \geq \limsup _{k \rightarrow \infty} \sum_{j=0}^{n_{k}-1} f\left(\sigma^{j}\left(\alpha_{k}\right)\right)-n_{k} \operatorname{Var}_{l+1}(f) .
\end{aligned}
$$

Seja $\tau_{k}$ o ramo inverso de $\sigma^{m_{k}-n_{k}}$ tal que $\tau_{k}\left(\sigma^{m_{k}}\left(\alpha_{k}\right)\right)=\sigma^{n_{k}}\left(\alpha_{k}\right)$. Seja $a_{k}:=\tau_{k}(a)$. Assim $\sigma^{m_{k}-n_{k}}\left(a_{k}\right)=a$.

$$
\begin{aligned}
d\left(b, a_{k}\right) & \leq d\left(b, \sigma^{n_{k}}\left(\alpha_{k}\right)\right)+d\left(\sigma^{n_{k}}\left(\alpha_{k}\right), a_{k}\right) \\
& \leq d\left(b, \sigma^{n_{k}}\left(\alpha_{k}\right)\right)+r^{m_{k}-n_{k}} d\left(\sigma^{m_{k}}\left(\alpha_{k}\right), a\right) \\
& \leq d\left(b, \sigma^{n_{k}}\left(\alpha_{k}\right)\right)+d\left(\sigma^{m_{k}}\left(\alpha_{k}\right), a\right) \rightarrow 0 \quad(k \rightarrow \infty) .
\end{aligned}
$$

Como $\lim _{k \rightarrow \infty} \alpha_{k}=a$ e $\lim _{k \rightarrow \infty} d\left(\sigma^{m_{k}}\left(\alpha_{k}\right), \alpha_{k}\right)=0$ então, existem $s, p \geq 1$ (assumiremos que $s \leq p$ ) tal que para $k$ suficientemente grande

$$
d\left(\sigma^{m_{k}}\left(\alpha_{k}\right), \alpha_{k}\right) \leq \frac{r^{s}}{2} \text { e } d\left(\alpha_{k}, a\right) \leq \frac{r^{p}}{2} .
$$


Desta forma

$$
\begin{aligned}
d\left(\sigma^{n_{k}}\left(\alpha_{k}\right), a_{k}\right) & \leq r^{m_{k}-n_{k}} d\left(\sigma^{m_{k}}\left(\alpha_{k}\right), a\right) \\
& \leq r^{m_{k}-n_{k}}\left(d\left(\sigma^{m_{k}}\left(\alpha_{k}\right), \alpha_{k}\right)+d\left(\alpha_{k}, a\right)\right) \\
& \leq r^{m_{k}-n_{k}+s} .
\end{aligned}
$$

Portanto

$$
\begin{aligned}
\left|\sum_{j=0}^{m_{k}-n_{k}-1} f\left(\sigma^{j}\left(a_{k}\right)\right)-\sum_{j=n_{k}}^{m_{k}-1} f\left(\sigma^{j}\left(\alpha_{k}\right)\right)\right| & \leq \operatorname{Var}_{m_{k}-n_{k}+s}(f)+\cdots+\operatorname{Var}_{s+1}(f) \\
& \leq\left(m_{k}-n_{k}-1\right) \operatorname{Var}_{s+1}(f)
\end{aligned}
$$

Assim

$$
\begin{aligned}
S_{f}(b, a) & \geq \limsup _{k \rightarrow \infty} \sum_{j=0}^{m_{k}-n_{k}-1} f\left(\sigma^{j}\left(a_{k}\right)\right) \\
& \geq \limsup _{k \rightarrow \infty} \sum_{j=n_{k}}^{m_{k}-1} f\left(\sigma^{j}\left(\alpha_{k}\right)\right)-\left(m_{k}-n_{k}-1\right) \operatorname{Var}_{s+1}(f),
\end{aligned}
$$

$\log 0$

$$
\begin{aligned}
0 \geq S_{f}(a, a) & \geq S_{f}(a, b)+S_{f}(b, a) \quad(\operatorname{item}(b)) \\
& \geq \limsup _{k \rightarrow \infty} \sum_{j=0}^{n_{k}-1} f\left(\sigma^{j}\left(\alpha_{k}\right)\right)+\limsup _{k \rightarrow \infty} \sum_{j=n_{k}}^{m_{k}-1} f\left(\sigma^{j}\left(\alpha_{k}\right)\right)-\left(m_{k}-n_{k}-1\right) \operatorname{Var}_{s+1}(f)-n_{k} \operatorname{Var}_{l+1}(f) \\
& \geq \limsup _{k \rightarrow \infty}\left[\sum_{j=0}^{n_{k}-1} f\left(\sigma^{j}\left(\alpha_{k}\right)\right)+\sum_{j=n_{k}}^{m_{k}-1} f\left(\sigma^{j}\left(\alpha_{k}\right)\right)\right](s, l \rightarrow \infty) \\
& \geq \limsup _{k \rightarrow \infty}\left[\sum_{j=0}^{m_{k}-1} f\left(\sigma^{j}\left(\alpha_{k}\right)\right)\right]=0 .
\end{aligned}
$$

$(\mathbf{j})$. Seja $\tau_{k}$ o ramo inverso de $\sigma^{n_{k}}$ tal que $\tau_{k}\left(\sigma^{n_{k}}\left(x_{0}\right)\right)=x_{0}$. Seja $b_{k}=\tau_{k}(b)$ para $k$ suficientemente grande. Então

$$
d\left(x_{0}, b_{k}\right) \leq r^{n_{k}} d\left(\sigma^{n_{k}}\left(x_{0}\right), b\right) \rightarrow 0(k \rightarrow \infty) .
$$

Como $d\left(\sigma^{n_{k}}\left(x_{0}\right), b\right) \rightarrow 0$ temos que existe $p \geq 1$ tal que para $k$ suficientemente grande $d\left(\sigma^{n_{k}}\left(x_{0}\right), b\right) \leq r^{p}, \operatorname{assim} d\left(x_{0}, b_{k}\right) \leq r^{n_{k}+p}$.

Deste modo

$$
\begin{aligned}
\left|\sum_{i=0}^{n_{k}-1} f\left(\sigma^{i}\left(x_{0}\right)\right)-\sum_{i=0}^{n_{k}-1} f\left(\sigma^{i}\left(b_{k}\right)\right)\right| & \leq \operatorname{Var}_{n_{k}+p}(f)+\cdots+\operatorname{Var}_{p+1}(f) \\
& \leq n_{k} \operatorname{Var}_{p+1}(f)
\end{aligned}
$$


E como $\lim _{k \rightarrow \infty} b_{k}=x_{0}$ e $\sigma^{n_{k}}\left(b_{k}\right)=b$ então

$$
\begin{aligned}
S_{f}\left(x_{0}, b\right) & \geq \limsup _{k \rightarrow \infty} \sum_{i=0}^{n_{k}-1} f\left(\sigma^{i}\left(b_{k}\right)\right) \\
& \geq \limsup _{k \rightarrow \infty} \sum_{i=0}^{n_{k}-1} f\left(\sigma^{i}\left(x_{0}\right)\right)-n_{k} \operatorname{Var}_{p+1}(f)(p \rightarrow \infty) \\
& \geq \limsup _{k \rightarrow \infty} \sum_{i=0}^{n_{k}-1} f\left(\sigma^{i}\left(x_{0}\right)\right) \\
& =\limsup _{k \rightarrow \infty} S_{f}\left(x_{0}, \sigma^{n_{k}}\left(x_{0}\right)\right) \quad \text { (ver hipótese do teorema). }
\end{aligned}
$$

Agora para $l \in \mathbb{N}$, seja $b_{l} \in \Sigma_{A}(\mathbb{N})$ e $m_{l} \in \mathbb{N}$ tal que $\lim _{l \rightarrow \infty} b_{l}=x_{0}, \sigma^{m_{l}}\left(b_{l}\right)=b$ e

$$
\lim _{l \rightarrow \infty} \sum_{j=0}^{m_{l}-1} f\left(\sigma^{j}\left(b_{l}\right)\right)=S_{f}\left(x_{0}, b\right) .
$$

Seja $\hat{\tau}_{l}$ o ramo inverso de $\sigma^{m_{l}}$ tal que $\hat{\tau}_{l}(b)=b_{l}$. Seja $x_{l}:=\hat{\tau}_{l}\left(\sigma^{n_{k}}\left(x_{0}\right)\right)$. Então

$$
\begin{aligned}
d\left(x_{l}, x_{0}\right) & \leq d\left(x_{0}, b_{l}\right)+d\left(b_{l}, x_{l}\right) \\
& \leq d\left(x_{0}, b_{l}\right)+r^{m_{l}} d\left(\sigma^{n_{k}}\left(x_{0}\right), b\right) \rightarrow 0(l \rightarrow \infty) .
\end{aligned}
$$

Como $\sigma^{n_{k}}\left(x_{0}\right) \rightarrow b$ então existe $s \geq 1$ tal que para $k$ suficientemente grande $d\left(\sigma^{n_{k}}\left(x_{0}\right), b\right) \leq r^{s}$ e assim

$$
d\left(b_{l}, x_{l}\right) \leq r^{m_{l}} d\left(\sigma^{m_{l}}\left(x_{l}\right), \sigma^{m_{l}}\left(b_{l}\right)\right)=r^{m_{l}} d\left(\sigma^{n_{k}}\left(x_{0}\right), b\right) \leq r^{m_{l}+s} .
$$

Portanto

$$
\begin{aligned}
\left|\sum_{j=0}^{m_{l}-1} f\left(\sigma^{j}\left(x_{l}\right)\right)-\sum_{j=0}^{m_{l}-1} f\left(\sigma^{j}\left(b_{l}\right)\right)\right| & \leq \operatorname{Var}_{m_{l}+s}(f)+\cdots+\operatorname{Var}_{s+1}(f) \\
& \leq m_{l} \operatorname{Var}_{s+1}(f)
\end{aligned}
$$

Já que $x_{l} \rightarrow x_{0}$ e $\sigma^{m_{l}}\left(x_{l}\right)=\sigma^{n_{k}}\left(x_{0}\right)$, temos que

$$
\begin{aligned}
S_{f}\left(x_{0}, \sigma^{n_{k}}\left(x_{0}\right)\right) & \geq \limsup _{l \rightarrow \infty} \sum_{j=0}^{m_{l}-1} f\left(\sigma^{j}\left(x_{l}\right)\right) \\
& \geq \limsup _{l \rightarrow \infty} \sum_{j=0}^{m_{l}-1} f\left(\sigma^{j}\left(b_{l}\right)\right)-m_{l} \operatorname{Var}_{s+1}(f)(s \rightarrow \infty) \\
& \geq S_{f}\left(x_{0}, b\right) .
\end{aligned}
$$

Deste modo

$$
\liminf _{k \rightarrow \infty} S_{f}\left(x_{0}, \sigma^{n_{k}}\left(x_{0}\right)\right) \geq S_{f}\left(x_{0}, b\right) \geq \limsup _{k \rightarrow \infty} S_{f}\left(x_{0}, \sigma^{n_{k}}\left(x_{0}\right)\right) .
$$

Neste ponto o leitor já deve conhecer um pouco sobre sub-ações e a relevância delas na Otimização Ergódica. Também já vimos vários exemplos de sub-ações calibradas, agora, uma pergunta natural que surge é a seguinte: existe alguma relação entre as sub-ações construídas até agora? A seguinte proposição responde afirmativamente a dita questão. Esta proposição generaliza o mesmo resultado obtido para shifts compactos em [CLT01]: 
Proposição 7. Seja $\left(\Sigma_{A}(\mathbb{N}), \sigma\right)$ um shift finitamente primitivo. E seja $f: \Sigma_{A}(\mathbb{N}) \rightarrow \mathbb{R}$ com variação somável e $P(f)<\infty$. Assuma que existe uma única medida maximizante $\mu$. Então para qualquer $a \in \operatorname{supp}(\mu)$ e $V$ é sub-ação calibrada contínua e limitada, temos que

$$
V(x)=V(a)+S_{f}(a, x) \text { para todo } x \in \Sigma_{A}(\mathbb{N}) .
$$

Demonstração. Tome $a \in \operatorname{supp}(\mu)$ fixo. Defina $R=f+V-V \circ \sigma-m(f)$. Por definição de sub-ação calibrada temos que $R \leq 0$. Dado $x_{0} \in \Sigma_{A}(\mathbb{N})$, considere uma sequência de pontos $x_{n}$ tais que $\sigma\left(x_{n}\right)=x_{n-1}$ e $R\left(x_{n}\right)=0, n=1,2, \ldots$ (esta sequência existe pois $V$ é calibrada) e defina uma sequência de probabilidades $\nu_{n}$ por $\nu_{n}=\frac{\delta_{x_{1}}+\cdots+\delta_{x_{n}}}{n}$. Usando que $V$ é limitada e $f$ é coerciva, existe um conjunto finito $B \subset \mathbb{N}$ tais que qualquer $x_{n}$ é da forma $b_{n} \ldots b_{1} x_{0}, b_{i} \in B, n \geq 1$. Isto mostra que existe pelo menos uma subsequência de $\left(\nu_{n}\right)_{n}$ convergindo para uma medida $\nu$ na topologia fraca*. Também temos que $\nu$ é $\sigma$-invariante e $\int R d \nu=0$ (a prova é a mesma da Afirmação 1 da seção 2.1). Então $\nu$ é $f$-maximizante, em particular $\nu=\mu$ e concluímos que $\mu$ é uma medida de acumulação de $\nu_{n}$. Segue que, para $n$ suficientemente grande, $\nu_{n}(\{z: d(z, a) \leq \epsilon\})>0$, pois $a \in \operatorname{supp}(\mu)$. Então $a$ é um ponto de acumulação da sequência $\left(x_{n}\right)_{n}$. Escrevendo $x_{n_{k}} \rightarrow a$, obtemos

$$
\begin{aligned}
V\left(x_{0}\right)-V(a) \geq S_{f}\left(a, x_{0}\right) & \geq \lim _{n_{k} \rightarrow \infty} S_{n_{k}}(f)\left(x_{n_{k}}\right)-n_{k} m(f) \\
& =\lim _{n_{k} \rightarrow \infty} S_{n_{k}}(R)\left(x_{n_{k}}\right)-V\left(x_{n_{k}}\right)+V\left(x_{0}\right) \\
& =V\left(x_{0}\right)-V(a) .
\end{aligned}
$$

Corolário 3. Em particular da Proposição acima, segue que duas sub-ações calibradas diferem por uma constante.

Demonstração. Sejam $V_{1}$ and $V_{2}$ duas sub-ações calibradas diferentes. Pela Proposition 7, temos que para todo $x \in \Sigma_{A}(\mathbb{N})$ e $a \in \operatorname{supp}(\mu)$

$$
\begin{aligned}
& V_{1}(x)=V_{1}(a)+S_{f}(a, x), \\
& V_{2}(x)=V_{2}(a)+S_{f}(a, x)
\end{aligned}
$$

$\operatorname{assim} V_{1}(x)-V_{2}(x)=V_{1}(a)-V_{2}(a)$ para todo $x \in \Sigma_{A}(\mathbb{N})$.

Como veremos no capítulo 3, a definição da função taxa ou função desvio $I$, vem dada em termos de uma sub-ação calibrada $V$. Assim provado que, duas duas sub-ações calibradas diferem por uma constante, garantimos que esta definição de $I$ é independe da escolha da sub-ação calibrada $V$.

A seguinte proposição mostra que qualquer sub-ação calibrada $V$, associada a um potencial $f$ com variação somável, é caracterizada por seus valores no Aubry e a Barreira de Peierls.

Proposição 8. Seja $\left(\Sigma_{A}(\mathbb{N}), \sigma\right)$ um shift finitamente primitivo e $f: \Sigma_{A}(\mathbb{N}) \rightarrow \mathbb{R}$ com variação somável e pressão topológica finita. Seja $V$ uma sub-ação calibrada uniformemente contínua e limitada, então para todo $y \in \Sigma_{A}(\mathbb{N})$ temos que

$$
V(y)=\sup _{x \in \Omega(f, \sigma)}\left[h_{f}(x, y)+V(x)\right] .
$$

Demonstração. Seja $\rho>0, x \in \Omega(f, \sigma)$ e $y, z \in \Sigma_{A}(\mathbb{N})$ tais que $\sigma^{n}(z)=y$. Tomar $\epsilon \in(0, \rho)$ tal que $d(x, z)<\epsilon \Rightarrow|V(x)-V(z)|<\rho$, para todo $x, z \in \Sigma_{A}(\mathbb{N})$. Então

$$
V(x)-V(y)=V(x)-V\left(\sigma^{n}(z)\right)<V(z)+\rho-V\left(\sigma^{n}(z)\right),
$$


assim

$$
V(x)-V(y)-\rho<V(z)-V\left(\sigma^{n}(z)\right) \leq-S_{n}(f-m(f))(z),
$$

portanto

$$
S_{n}(f-m(f))(z) \leq V(y)-V(x)+\rho,
$$

logo, tomando o supremo sobre todos os $z$ tales que $d(x, z)<\epsilon$ e $\sigma^{n}(z)=y$ e o limsup quando $n \rightarrow \infty$ obtemos

$$
\limsup _{n \rightarrow \infty} \sup _{z} S_{n}(f-m(f))(z) \leq V(y)-V(x)+\rho,
$$

fazendo $\rho \rightarrow 0$ segue que

$$
h_{f}(x, y) \leq V(y)-V(x)
$$

por conseguinte

$$
\sup _{x \in \Omega(f, \sigma)}\left\{V(x)+h_{f}(x, y)\right\} \leq V(y) .
$$

Agora provaremos a outra implicação. Com efeito, seja $\epsilon>0$ dado, e $x_{0} \in \Sigma_{A}(\mathbb{N})$.

Defina $R:=f+V-V \circ \sigma-m(f)$. como $V$ é uma sub-ação calibrada, existe uma sequência de pontos $\left(x_{n}\right)_{n}$ tais que $\sigma\left(x_{n}\right)=x_{n-1}$ e $R\left(x_{n}\right)=0, n=1,2, \ldots$

Usando que $V$ é limitada e $f$ é coerciva, existe um subconjunto finito $B \subset \mathbb{N}$ tal que todos os $x_{n}$ 's são da forma $b_{n} \ldots b_{1} x_{0}, b_{i} \in B, n \geq 1$. Seja $a$ um ponto de acumulação da sequência $\left(x_{n}\right)_{n}$.

Provaremos que $a \in \Omega(f, \sigma)$.

Afirmação 4. $S_{f}(a, a)=0$.

Com efeito:

Seja $k_{l}$ uma sequência crescente qualquer. Seja $m_{l}=k_{l+1}-k_{l}$. Então $\sigma^{m_{l}}\left(x_{k_{l}+1}\right)=x_{k_{l}}$. Seja $\tau_{k_{l}}$ um ramo inverso de $\sigma^{m_{l}}$ tal que $\tau_{k_{l}}\left(x_{k_{l}}\right)=x_{k_{l}+1}$ e $a_{k_{l}+1}:=\tau_{k_{l}}(a)$. Já que $x_{k_{l}} \rightarrow a$, segue que

$$
\begin{aligned}
d\left(a_{k_{l}+1}, a\right) & \leq d\left(a_{k_{l}+1}, x_{k_{l}+1}\right)+d\left(x_{k_{l}+1}, a\right) \\
& \leq r^{m_{l}} d\left(a, x_{k_{l}}\right)+d\left(x_{k_{l}+1}, a\right) \\
& \leq d\left(a, x_{k_{l}}\right)+d\left(x_{k_{l}+1}, a\right) \rightarrow 0(l \rightarrow \infty) .
\end{aligned}
$$

Além disso, já que $\lim _{l \rightarrow \infty} x_{k_{l}}=a$, então existe $s \geq 1$ tal que $d\left(x_{k_{l}}, a\right) \leq r^{s}$ para todo $l$, assim obtemos

$$
\begin{aligned}
d\left(a_{k_{l}+1}, x_{k_{l}+1}\right) & \leq r^{m_{l}} d\left(a, x_{k_{l}}\right) \\
& \leq r^{m_{l}} r^{s}=r^{m_{l}+s} .
\end{aligned}
$$

Então

$$
\begin{aligned}
\left|\sum_{j=0}^{m_{l}-1} f\left(\sigma^{j}\left(a_{k_{l}+1}\right)\right)-\sum_{j=0}^{m_{l}-1} f\left(\sigma^{j}\left(x_{k_{l}+1}\right)\right)\right| & \leq \operatorname{Var}_{m_{l}+s}(f)+\cdots+\operatorname{Var}_{s+1}(f) \\
& \leq m_{l} \operatorname{Var}_{s+1}(f) .
\end{aligned}
$$

Portanto

$$
\begin{aligned}
0 \geq S_{f}(a, a) & \geq \limsup _{l \rightarrow \infty} \sum_{j=0}^{m_{l}-1} f\left(\sigma^{j}\left(a_{k_{l}+1}\right)\right) \\
& \geq \limsup _{l \rightarrow \infty} \sum_{j=0}^{m_{l}-1} f\left(\sigma^{j}\left(x_{k_{l}+1}\right)\right)-m_{l} \operatorname{Var}_{s+1}(f) \quad(s \rightarrow \infty) \\
& \geq \limsup _{l \rightarrow \infty}\left(V\left(x_{k_{l}}\right)-V\left(x_{k_{l}+1}\right)\right)=0 .
\end{aligned}
$$


Portanto $a \in \Omega(f, \sigma)$. Por outro lado note que

$$
S_{n_{k}}(f-m(f))\left(x_{n_{k}}\right)=V\left(x_{0}\right)-V\left(x_{n_{k}}\right) .
$$

Assim da equação acima, a continuidade de $V$ e que $x_{n_{k}} \rightarrow a$ obtemos

$$
h_{f}\left(a, x_{0}\right) \geq V\left(x_{0}\right)-V(a),
$$

$\log 0$

$$
V\left(x_{0}\right) \leq \sup _{a \in \Omega(f, \sigma)}\left\{h_{f}\left(a, x_{0}\right)+V(a)\right\} .
$$

Corolário 4. Seja $V, V^{\prime} \in C^{0}\left(\Sigma_{A}(\mathbb{N})\right)$ sub-ações para o potencial $f$. Se $V \leq V^{\prime}$ in $\Omega(f, \sigma)$, então $V \leq V^{\prime}$ em $\Sigma_{A}(\mathbb{N})$. Em particular, igualdade em $\Omega(f, \sigma)$ implica igualdade em $\Sigma_{A}(\mathbb{N})$. 


\section{Capítulo 4}

\section{Princípio dos Grandes Desvios à Temperatura Zero}

Nesse capítulo apresentamos a demonstração do teorema principal. Isto é, mostramos que a familia de estados Gibbs $\left(\mu_{\beta}\right)_{\beta}$ satisfaz um princípio dos grandes desvios:

Teorema 16. Sejam $\left(\Sigma_{A}(\mathbb{N}), \sigma\right)$ um shift finitamente primitivo e $f: \Sigma_{A}(\mathbb{N}) \rightarrow \mathbb{R}$ com variação somável e satisfazendo $P(f)<\infty$, além disso assuma que $f$ admite uma única medida maximizante $\mu$. Para cada $\beta$, denotamos $\mu_{\beta}$, a única medida de equilíbrio associada a $\beta f$. Então $\left(\mu_{\beta}\right)_{\beta}$ satisfaz um Principio dos Grandes Desvios em cilindros, isto é, para qualquer cilindro $C=\left[x_{0} \ldots x_{n-1}\right]$ de $\Sigma_{A}(\mathbb{N})$,

$$
\lim _{\beta \rightarrow \infty} \frac{1}{\beta} \log \mu_{\beta}(C)=-\inf _{x \in C} I(x)
$$

onde

$$
I(x)=\sum_{j=0}^{\infty}(V \circ \sigma-V-f+m(f)) \circ \sigma^{j}(x), \quad m(f)=\int f d \mu
$$

onde $V$ é uma sub-ação calibrada para $f$.

\subsection{Princípio dos Grandes Desvios}

Nesta seção provaremos um princípio dos grandes desvios para $\left(\mu_{\beta}\right)_{\beta}$ nos cilindros. Uma boa referência para aprofundar mais na área de grandes desvios e aplicações é [DZ98, El106, OV04].

Ao longo deste seção, assumiremos que $\left(\Sigma_{A}(\mathbb{N}), \sigma\right)$ é um shift finitamente primitivo e $f: \Sigma_{A}(\mathbb{N}) \rightarrow \mathbb{R}$ tem variação somável e $P(f)<\infty$.

Definição 25. Dizemos que a família de probabilidades $\left(\mu_{\beta}\right)_{\beta}$ satisfaz um princípio dos grandes desvios com função de desvio $I$, se para todo cilindro $C \subseteq \Sigma_{A}(\mathbb{N})$

$$
\lim _{\beta \rightarrow \infty} \frac{1}{\beta} \log \mu_{\beta}(C)=-\inf _{x \in C} I(x)
$$

para alguma função $I \geq 0$ semicontínua inferiormente.

Defina

$$
g_{\beta}:=\beta f+\log h_{\beta}-\log \left(h_{\beta} \circ \sigma\right)-P(\beta f) .
$$

Note que $g_{\beta}$ é limitada superiormente e $L_{g_{\beta}} 1=1$. Como, $\frac{1}{\beta} \log h_{\beta} \rightarrow V$ (uniformemente em compactos ), onde $V$ é uma sub-ação calibrada (veja teorema 14), assim

$$
\frac{g_{\beta}}{\beta} \rightarrow f+V-V \circ \sigma-m(f) .
$$


Definimos $R:=V \circ \sigma-V-f+m(f)$. Note que $R \geq 0$ pois $V$ é uma sub-ação calibrada. Assim $g_{\beta} / \beta \rightarrow-R$ uniformemente em compactos.

Agora, estamos interessados na existência do seguinte limite

$$
\lim _{\beta \rightarrow \infty} \frac{1}{\beta} \log \mu_{\beta}(C), \text { para todo cilindro } C=\left[x_{0}, \ldots, x_{n-1}\right] .
$$

Lema 12. Para todo cilindro $C$ a familia $\frac{1}{\beta} \log \mu_{\beta}(C)$ é limitada.

Demonstração. Seja $C=\left[x_{0} \ldots x_{m}\right]$ para algum $m \in \mathbb{N}$. Da equação $(2.6)$ e o fato que

$$
P(\beta f) \leq \beta P(f), \forall \beta \geq 1,
$$

segue que

$$
e^{\eta_{1} \beta} \leq \mu_{\beta}\left[x_{0} \ldots x_{m}\right] \leq e^{\eta_{2} \beta} e^{-(m+1) P(\beta f)} .
$$

Onde

$$
\begin{gathered}
\eta_{1}:=-4 \operatorname{Var}(f)+\left.\inf f\right|_{\left[x_{0}\right]}+\cdots+\left.\inf f\right|_{\left[x_{m}\right]}-(m+1) P(f) . \\
\eta_{2}:=4 \operatorname{Var}(f)+\left.\sup f\right|_{\left[x_{0}\right]}+\cdots+\sup f_{\left[x_{m}\right]} .
\end{gathered}
$$

Considere $\nu \in \mathcal{M}_{\sigma}\left(\Sigma_{A}(\mathbb{N})\right)$ qualquer medida tais que $L:=\int f d \nu$ é finita (poderia tomar por exemplo $\nu$ suportada em uma órbita periódica). Assim, usando que $P(f+\alpha)=P(f)+\alpha$, com $\alpha \in \mathbb{R}$ (ver [Wal82]), obtemos que:

$$
P(\beta f)-\beta L=P(\beta(f-L)) \geq \int \beta(f-L) d \nu+h(\nu) \geq 0 .
$$

(Lembre que $h(\nu)$ é a entropia da medida $\nu$ ) Logo

$$
\begin{aligned}
e^{\eta_{2} \beta} e^{-(m+1) P(\beta f)} & =e^{\eta_{2} \beta} e^{-\beta(m+1) L} e^{-(m+1) P(\beta(f-L))} \\
& \leq e^{\eta_{2} \beta} e^{-\beta(m+1) L} .
\end{aligned}
$$

Por conseguinte a equação (4.6) se transforma em:

$$
e^{\eta_{1} \beta} \leq \mu_{\beta}\left[x_{0} \ldots x_{m}\right] \leq e^{\eta_{2} \beta} e^{-\beta(m+1) L} .
$$

desta forma temos que

$$
\eta_{1} \leq \frac{1}{\beta} \log \mu_{\beta}\left[x_{0} \ldots x_{m}\right] \leq \eta_{2}-(m+1) L .
$$

Procedendo por indução concluímos que $\frac{1}{\beta} \log \mu_{\beta}(C)$ está uniformemente limitada para todo cilindro $C$.

Usando o fato que os cilindros são enumeráveis, tomar $C_{1}, C_{2}, \ldots$ uma enumeração dos cilindros. Tomar uma subsequência $\beta_{11}, \beta_{12}, \beta_{13}, \ldots$ tal que o limite (4.5) existe para $C_{1}$, logo tomar uma subsequência desta, denotada por $\beta_{21}, \beta_{22}, \ldots$ tal que o limite (4.5) existe para $C_{2}$, logo tomar uma subsequência desta última, denotada por $\beta_{31}, \beta_{32}, \ldots$ tal que o limite (4.5) existe para $C_{3}$, etc. Depois tomar uma sequência $\beta_{11}, \beta_{22}, \beta_{33}, \ldots$ (diagonal) assim o limite existe para cada cilindro $C$. Denotando esta sequência (diagonal) por $\left(\beta_{i}\right)_{i}$, obtemos que

$$
\lim _{\beta_{i} \rightarrow \infty} \frac{1}{\beta_{i}} \log \mu_{\beta_{i}}(C), \text { existe para todo cilindro } C \text {. }
$$

Tomamos esta subsequência da sequência que já sabemos que vale $\lim _{\beta_{n} \rightarrow \infty} \frac{1}{\beta_{n}} \log h_{\beta_{n}}$ e as demais construções feitas até aqui. 
No caso específico de potenciais que depende de 2 coordenadas o fato de sabermos que existe o limite $\lim _{\beta \rightarrow \infty} \frac{1}{\beta} \log \mu_{\beta}\left[x_{0}\right]$ implica a existência do limite $\lim _{\beta \rightarrow \infty} \frac{1}{\beta} \log \mu_{\beta}[\gamma]$ para qualquer ciclo $\gamma=x_{0} \cdots x_{k} \operatorname{com} x_{k}=x_{0}$. A proposição à seguir mostra isso

Proposição 9. Seja $\Sigma_{A}(\mathbb{N})$ um shift topologicamente mixing com a propriedade BIP e seja $f: \Sigma_{A}(\mathbb{N}) \rightarrow \mathbb{R}$ dependo de finitas coordenadas com variação somável e pressão finita, então

$$
\lim _{\beta \rightarrow \infty} \frac{\log \mu_{\beta f}[\gamma]}{\beta} \text { existe se e somente se } \lim _{\beta \rightarrow \infty} \frac{\log \mu_{\beta f}\left[x_{1}\right]}{\beta} \text { existe }
$$

(aqui $\gamma$ é um ciclo $\gamma=x_{1} \cdots x_{n}$ com $x_{n}=x_{1}$ )

Demonstração. Pela propriedade de Markov de medidas de Gibbs associada ao potencial $f$ com $f(x)=f\left(x_{0} x_{1}\right)$ garante que para um ciclo $\gamma=x_{1} \cdots x_{n} \operatorname{com} x_{n}=x_{1}$ temos que

$$
\mu_{\beta f}[\gamma]=\mu_{\beta f}\left[x_{1}\right] \exp \left(\beta S_{n} f(\bar{x})-n P(\beta f)\right)
$$

donde $\bar{x}$ é um elemento do cilindro $\left[x_{1} \ldots x_{n}\right]$, assim

$$
\begin{aligned}
\lim _{\beta \rightarrow \infty} \frac{\log \mu_{\beta f}[\gamma]}{\beta} & =\lim _{\beta \rightarrow \infty} \frac{\log \mu_{\beta f}\left[x_{1}\right]}{\beta}+S_{n} f(\bar{x})-n \lim _{\beta \rightarrow \infty} \frac{P(\beta f)}{\beta} \\
& =\lim _{\beta \rightarrow \infty} \frac{\log \mu_{\beta f}\left[x_{1}\right]}{\beta}+S_{n} f(\bar{x})-n m(f) .
\end{aligned}
$$

No seguinte teorema provaremos um princípio dos grandes desvios, com uma função taxa diferente à função taxa do Teorema 16, depois provaremos que estas duas funções são iguais (veja proposição 12).

Teorema 17. Seja $\left(\Sigma_{A}(\mathbb{N}), \sigma\right)$ um shift finitamente primitivo. Seja $f: \Sigma_{A}(\mathbb{N}) \rightarrow \mathbb{R}$ uma função com variação somável e $P(f)<\infty$. Suponha também que $\lim _{\beta_{i} \rightarrow \infty} \frac{1}{\beta_{i}} \log \mu_{\beta_{i}}$ existe para qualquer cilindro $C$, então

1. A função

$$
\tilde{I}(x):=-\lim _{n \rightarrow \infty} \lim _{\beta_{i} \rightarrow \infty} \frac{1}{\beta_{i}} \log \mu_{\beta_{i}}\left(\left[x_{0} \ldots x_{n-1}\right]\right) \geq 0
$$

está bem definida e é semi-continua inferiormente.

2.

$$
\lim _{\beta_{i} \rightarrow \infty} \frac{1}{\beta_{i}} \log \mu_{\beta_{i}}(C)=-\inf _{x \in C} \tilde{I}(x) \text { para todo cilindro } C \text {. }
$$

3. $\tilde{I}(x) \geq \tilde{I}(\sigma(x))$. Em particular, existe $\tilde{I}_{0}(x):=\lim _{n \rightarrow \infty} \tilde{I}\left(\sigma^{n}(x)\right), \forall x \in \Sigma_{A}(\mathbb{N})$.

\section{Demonstração.}

\section{Prova de 1}

Fixado $x=x_{0} x_{1} \ldots$ temos que

$$
\lim _{\beta_{i} \rightarrow \infty} \frac{1}{\beta_{i}} \log \left(\mu_{\beta_{i}}\left[x_{0} \ldots x_{n}\right]\right)
$$

é decrescente em $n$, assim existe

$$
\tilde{I}(x):=-\lim _{n \rightarrow \infty} \lim _{\beta_{i} \rightarrow \infty} \frac{1}{\beta_{i}} \log \left(\mu_{\beta_{i}}\left[x_{0} \ldots x_{n}\right]\right) .
$$

e $\tilde{I}: \Sigma_{A}(\mathbb{N}) \rightarrow[0, \infty]$.

$\tilde{I}$ é semi-contínua inferiormente 
Com efeito, fixado $z=x_{0} x_{1} \ldots$ só precisamos provar que

$$
\liminf _{m \rightarrow \infty} \inf _{z_{m} \in\left[x_{0} \ldots x_{m}\right]} \tilde{I}\left(z_{m}\right) \geq \tilde{I}(z)
$$

pois $\tilde{I}\left[x_{0} \ldots x_{m}\right] \geq \inf _{z_{m} \in\left[x_{0} \ldots x_{m}\right]} \tilde{I}\left(z_{m}\right)$. Se denotamos $z_{m}=x_{0} \ldots x_{m} y_{0}^{m} y_{1}^{m} \ldots$ a equação acima é equivalente a

$$
\liminf _{m \rightarrow \infty} \inf _{z_{m} \in\left[x_{0} \ldots x_{m}\right]}-\lim _{n \rightarrow \infty} \lim _{\beta_{i} \rightarrow \infty} \frac{1}{\beta_{i}} \log \left(\mu_{\beta_{i}}\left[x_{0} \ldots x_{m} y_{0}^{m} y_{1}^{m} \ldots y_{n}^{m}\right]\right) \geq \tilde{I}(z) .
$$

Isto é

$$
\liminf _{m \rightarrow \infty}-\sup _{z_{m} \in\left[x_{0} \ldots x_{m}\right]} \lim _{n \rightarrow \infty} \lim _{\beta_{i} \rightarrow \infty} \frac{1}{\beta_{i}} \log \left(\mu_{\beta_{i}}\left[x_{0} \ldots x_{m} y_{0}^{m} y_{1}^{m} \ldots y_{n}^{m}\right]\right) \geq \tilde{I}(z)
$$

$\mathrm{Ou}$

$$
-\limsup _{m \rightarrow \infty} \sup _{z_{m} \in\left[x_{0} \ldots x_{m}\right]} \lim _{n \rightarrow \infty} \lim _{\beta_{i} \rightarrow \infty} \frac{1}{\beta_{i}} \log \left(\mu_{\beta_{i}}\left[x_{0} \ldots x_{m} y_{0}^{m} y_{1}^{m} \ldots y_{n}^{m}\right]\right) \geq \tilde{I}(z)
$$

$\mathrm{Ou}$

$$
\limsup _{m \rightarrow \infty} \sup _{z_{m} \in\left[x_{0} \ldots x_{m}\right]} \lim _{n \rightarrow \infty} \lim _{\beta_{i} \rightarrow \infty} \frac{1}{\beta_{i}} \log \left(\mu_{\beta_{i}}\left[x_{0} \ldots x_{m} y_{0}^{m} y_{1}^{m} \ldots y_{n}^{m}\right]\right) \leq-\tilde{I}(z) .
$$

Assim provar que $\tilde{I}$ é semi-contínua inferiormente é equivalente a provar (4.13). Deste modo concluímos a prova observando que

$$
\begin{aligned}
& \limsup _{m \rightarrow \infty} \sup _{z_{m} \in\left[x_{0} \ldots x_{m}\right]} \lim _{n \rightarrow \infty} \lim _{\beta_{i} \rightarrow \infty} \frac{1}{\beta_{i}} \log \left(\mu_{\beta_{i}}\left[x_{0} \ldots x_{m} y_{0}^{m} y_{1}^{m} \ldots y_{n}^{m}\right]\right) \\
& \leq \limsup _{m \rightarrow \infty} \sup _{z_{m} \in\left[x_{0} \ldots x_{m}\right]} \lim _{n \rightarrow \infty} \lim _{\beta_{i} \rightarrow \infty} \frac{1}{\beta_{i}} \log \left(\mu_{\beta_{i}}\left[x_{0} \ldots x_{m}\right]\right) \\
& \leq \limsup _{m \rightarrow \infty} \lim _{\beta_{i} \rightarrow \infty} \frac{1}{\beta_{i}} \log \left(\mu_{\beta_{i}}\left[x_{0} \ldots x_{m}\right]\right) \\
& =\lim _{m \rightarrow \infty} \lim _{\beta_{i} \rightarrow \infty} \frac{1}{\beta_{i}} \log \left(\mu_{\beta_{i}}\left[x_{0} \ldots x_{m}\right]\right) \\
& =-\tilde{I}(z) .
\end{aligned}
$$

\section{Prova de 2}

$$
\lim _{\beta_{i} \rightarrow \infty} \frac{1}{\beta_{i}} \log \mu_{\beta_{i}}(C)=-\inf _{x \in C} \tilde{I}(x) .
$$

Seja $x \in C=\left[x_{0}, \ldots, x_{l}\right]$, então

$$
\begin{aligned}
\lim _{\beta_{i} \rightarrow \infty} \frac{1}{\beta_{i}} \log \mu_{\beta_{i}}(C) & =\lim _{n \rightarrow \infty} \lim _{\beta_{i} \rightarrow \infty} \frac{1}{\beta_{i}} \log \mu_{\beta_{i}}(C) \\
& \geq \lim _{n \rightarrow \infty} \lim _{\beta_{i} \rightarrow \infty} \frac{1}{\beta_{i}} \log \mu_{\beta_{i}}\left(\left[x_{0} \ldots x_{n}\right]\right) \\
& =-\tilde{I}(x),
\end{aligned}
$$

$\operatorname{logo}-\tilde{I}(x) \leq \lim _{\beta_{i} \rightarrow \infty} \frac{1}{\beta_{i}} \log \mu_{\beta_{i}}(C)$ assim $\sup _{x \in C}-\tilde{I}(x) \leq \lim _{\beta_{i} \rightarrow \infty} \frac{1}{\beta_{i}} \log \mu_{\beta_{i}}(C)$, portanto

$$
\lim _{\beta_{i} \rightarrow \infty} \frac{1}{\beta_{i}} \log \mu_{\beta_{i}}(C) \geq-\inf _{x \in C} \tilde{I}(x) .
$$

Agora provamos a outra desigualdade. Com efeito escrevemos $C=\left[x_{0} \ldots x_{m}\right]$ para algum $m \in \mathbb{N}$. 
Da equação (2.6) segue que

$$
e^{\eta_{1} \beta} e^{\left.\beta \inf f\right|_{[i]}} \leq \mu_{\beta}\left[x_{0} \ldots x_{m} i\right] \leq e^{\eta_{2} \beta} e^{\left.\beta \sup f\right|_{[i]}} e^{-(m+2) P(\beta f)} .
$$

Onde

$$
\begin{gathered}
\eta_{1}:=-4 \operatorname{Var}(f)+\left.\inf f\right|_{\left[x_{0}\right]}+\cdots+\left.\inf f\right|_{\left[x_{m}\right]}-(m+2) P(f) . \\
\eta_{2}:=4 \operatorname{Var}(f)+\left.\sup f\right|_{\left[x_{0}\right]}+\cdots+\sup f_{\left[x_{m}\right]} .
\end{gathered}
$$

Considere $\nu \in \mathcal{M}_{\sigma}\left(\Sigma_{A}(\mathbb{N})\right)$ qualquer medida tais que $L:=\int f d \nu$ é finita (poderia tomar por exemplo $\nu$ suportada em uma órbita periódica). Assim

$$
P(\beta f)-\beta L=P(\beta(f-L)) \geq \int \beta(f-L) d \nu+h(\nu) \geq 0 .
$$

Logo

$$
\begin{aligned}
e^{\eta_{2} \beta} e^{\left.\beta \sup f\right|_{[i]}} e^{-(m+2) P(\beta f)} & =e^{\eta_{2} \beta} e^{\beta\left(\left.\sup f\right|_{[i]}-(m+2) L\right)} e^{-(m+2) P(\beta(f-L))} \\
& \leq e^{\eta_{2} \beta} e^{\beta\left(\left.\sup f\right|_{[i]}-(m+2) L\right)} .
\end{aligned}
$$

Logo a equação (4.14) se transforma em:

$$
e^{\eta_{1} \beta} e^{\left.\beta \inf f\right|_{[i]}} \leq \mu_{\beta}\left[x_{0} \ldots x_{m} i\right] \leq e^{\eta_{2} \beta} e^{\beta\left(\left.\sup f\right|_{[i]}-(m+2) L\right)} .
$$

Já que $\sum_{i=0}^{\infty} e^{\left.\sup f\right|_{[i]}}<\infty$, existe $J$ (suficientemente grande) tal que

$$
\sum_{i \geq J} e^{\eta_{2}} e^{\left.\sup f\right|_{[i]}-(m+2) L} \leq e^{\eta_{1}} e^{\left.\inf f\right|_{[0]}}
$$

Portanto para $\beta>1$ obtemos

$$
\begin{aligned}
\sum_{i \geq J} \mu_{\beta}\left[x_{0} \ldots x_{m} i\right] & \leq \sum_{i \geq J} e^{\eta_{2} \beta} e^{\beta\left(\left.\sup f\right|_{[i]}-(m+2) L\right)} \\
& \leq\left(\sum_{i \geq J} e^{\eta_{2}} e^{\left.\sup f\right|_{[i]}-(m+2) L}\right)^{\beta} \\
& \leq e^{\eta_{1} \beta} e^{\left.\beta \inf f\right|_{[0]}} \\
& \leq \mu_{\beta}\left[x_{0} \ldots x_{m} 0\right] \\
& \leq \sum_{i=0}^{J-1} \mu_{\beta}\left[x_{0} \ldots x_{m} i\right] .
\end{aligned}
$$

Deste modo temos que

$$
\begin{aligned}
\mu_{\beta}\left[x_{0} \ldots x_{m}\right] & =\sum_{i=0}^{\infty} \mu_{\beta}\left[x_{0} \ldots x_{m} i\right] \\
& \leq 2 \sum_{i=0}^{J-1} \mu_{\beta}\left[x_{0} \ldots x_{m} i\right]
\end{aligned}
$$

portanto

$$
\lim _{\beta \rightarrow \infty} \frac{1}{\beta} \log \mu_{\beta}\left[x_{0} \ldots x_{m}\right] \leq \lim _{\beta \rightarrow \infty} \frac{1}{\beta} \log \sum_{i=0}^{J-1} \mu_{\beta}\left[x_{0} \ldots x_{m} i\right] .
$$


E usando a concavidade da função logaritmo obtemos que

$$
\lim _{\beta \rightarrow \infty} \frac{1}{\beta} \log \mu_{\beta}\left[x_{0} \ldots x_{m}\right] \geq \lim _{\beta \rightarrow \infty} \frac{1}{\beta} \log \sum_{i=0}^{J-1} \mu_{\beta}\left[x_{0} \ldots x_{m} i\right] .
$$

Em consequência

$$
\begin{aligned}
\lim _{\beta \rightarrow \infty} \frac{1}{\beta} \log \mu_{\beta}\left[x_{0} \ldots x_{m}\right] & =\max \left\{\lim _{\beta \rightarrow \infty} \frac{1}{\beta} \log \sum_{i \geq J} \mu_{\beta}\left[x_{0} \ldots x_{m} i\right], \lim _{\beta \rightarrow \infty} \frac{1}{\beta} \log \sum_{i=0}^{J-1} \mu_{\beta}\left[x_{0} \ldots x_{m} i\right]\right\} \\
& =\lim _{\beta \rightarrow \infty} \frac{1}{\beta} \log \sum_{i=0}^{J-1} \mu_{\beta}\left[x_{0} \ldots x_{m} i\right] \\
& =\max _{0 \leq i<J}\left\{\lim _{\beta \rightarrow \infty} \frac{1}{\beta} \log \mu_{\beta}\left[x_{0} \ldots x_{m} i\right]\right\} .
\end{aligned}
$$

Em particular, existe algum $x_{m+1} \in \mathbb{N}$ tais que

$$
\lim _{\beta \rightarrow \infty} \frac{1}{\beta} \log \mu_{\beta}\left[x_{0} \ldots x_{m}\right]=\lim _{\beta \rightarrow \infty} \frac{1}{\beta} \log \mu_{\beta}\left[x_{0} \ldots x_{m} x_{m+1}\right] .
$$

Desta maneira usando um argumento indutivo podemos construir uma sequência de símbolos $y_{0}, y_{1}, \ldots$ tais que

$$
\begin{aligned}
\lim _{\beta_{i} \rightarrow \infty} \frac{1}{\beta_{i}} \log \mu_{\beta_{i}}(C) & =\lim _{\beta_{i} \rightarrow \infty} \frac{1}{\beta_{i}} \log \mu_{\beta_{i}}\left(\left[x_{0} \ldots x_{m} y_{0}\right]\right) \\
& =\lim _{\beta_{i} \rightarrow \infty} \frac{1}{\beta_{i}} \log \mu_{\beta_{i}}\left(\left[x_{0} \ldots x_{m} y_{0} y_{1}\right]\right)=\cdots
\end{aligned}
$$

Seja $z$ o ponto de interseção do cilindro $\left[x_{0} \ldots x_{m} y_{0} \ldots y_{n}\right], n \geq 1$. Então, quando $n \rightarrow \infty$

$$
\begin{aligned}
\lim _{\beta_{i} \rightarrow \infty} \frac{1}{\beta_{i}} \log \mu_{\beta_{i}}(C) & =\lim _{n \rightarrow \infty} \lim _{\beta_{i} \rightarrow \infty} \frac{1}{\beta_{i}} \log \mu_{\beta_{i}}\left(\left[x_{0} \ldots x_{m} y_{0} \ldots y_{n}\right]\right) \\
& =-\tilde{I}(z) \\
& \leq-\inf _{z \in C} \tilde{I}(z) .
\end{aligned}
$$

\section{Prova de 3}

Para cada $n$

$$
\begin{aligned}
\lim _{\beta_{i} \rightarrow \infty} \frac{1}{\beta_{i}} \log \left(\mu_{\beta_{i}}\left[x_{0} \ldots x_{n}\right]\right) & \leq \lim _{\beta_{i} \rightarrow \infty} \frac{1}{\beta_{i}} \log \mu_{\beta_{i}}\left(\sigma^{-1}\left[x_{1} \ldots x_{n}\right]\right) \\
& =\lim _{\beta_{i} \rightarrow \infty} \frac{1}{\beta_{i}} \log \mu_{\beta_{i}}\left(\left[x_{1} \ldots x_{n}\right]\right) .
\end{aligned}
$$

Na igualdade usamos que $\mu_{\beta}$ é uma medida $\sigma$-invariante. Deste modo

$$
\begin{aligned}
\tilde{I}(x):=-\lim _{n \rightarrow \infty} \lim _{\beta_{i} \rightarrow \infty} \frac{1}{\beta_{i}} \log \left(\mu_{\beta_{i}}\left[x_{0} \ldots x_{n}\right]\right) & \geq-\lim _{n \rightarrow \infty} \lim _{\beta_{i} \rightarrow \infty} \frac{1}{\beta_{i}} \log \left(\mu_{\beta_{i}}\left[x_{1} \ldots x_{n}\right]\right) \\
& =: \tilde{I}(\sigma(x)) .
\end{aligned}
$$


Proposição 10. Se $\lim _{\beta \rightarrow \infty} \frac{g_{\beta}(x)}{\beta}=-R(x)$ então

$$
\lim _{n \rightarrow \infty} \lim _{\beta \rightarrow \infty} \frac{1}{\beta} \log \frac{\mu_{\beta}\left[x_{1} \ldots x_{n}\right]}{\mu_{\beta}\left[x_{0} \ldots x_{n}\right]}=R(x)
$$

Demonstração. Seja $\epsilon>0$ dado. Aplicando a Proposição 4 à função $g_{\beta}$, segue que

$$
\left(\inf _{x \in\left[x_{0} \ldots x_{n}\right]} e^{-g_{\beta}(x)}\right) \mu_{\beta}\left(\left[x_{0} \ldots x_{n}\right]\right) \leq \mu_{\beta}\left(\left[x_{1} \ldots x_{n}\right]\right) \leq\left(\sup _{x \in\left[x_{0} \ldots x_{n}\right]} e^{-g_{\beta}(x)}\right) \mu_{\beta}\left(\left[x_{0} \ldots x_{n}\right]\right)
$$

isto é equivalente a

$$
\inf _{x \in\left[x_{0} \ldots x_{n}\right]}-\frac{g_{\beta}(x)}{\beta} \leq \frac{1}{\beta} \log \frac{\mu_{\beta}\left[x_{1} \ldots x_{n}\right]}{\mu_{\beta}\left[x_{0} \ldots x_{n}\right]} \leq \sup _{x \in\left[x_{0} \ldots x_{n}\right]}-\frac{g_{\beta}(x)}{\beta}
$$

Seja $x$ o ponto de interseç̧ão dos cilindros $\left[x_{0} \ldots x_{n}\right]$ para todo $n$. Como $\frac{g_{\beta}}{\beta}$ tem a variação somável obtemos que

$$
\sup _{z \in\left[x_{0} \ldots x_{n}\right]}-\frac{g_{\beta}(z)}{\beta} \leq-\frac{g_{\beta}(x)}{\beta}+\operatorname{Var}_{n+1}\left(\frac{g_{\beta}}{\beta}\right),
$$

e

$$
\inf _{z \in\left[x_{0} \ldots x_{n}\right]}-\frac{g_{\beta}(z)}{\beta} \geq-\frac{g_{\beta}(x)}{\beta}-\operatorname{Var}_{n+1}\left(\frac{g_{\beta}}{\beta}\right),
$$

assim obtemos

$$
-\operatorname{Var}_{n+1}\left(\frac{g_{\beta}}{\beta}\right)-\frac{g_{\beta}(x)}{\beta} \leq \frac{1}{\beta} \log \frac{\mu_{\beta}\left[x_{1} \ldots x_{n}\right]}{\mu_{\beta}\left[x_{0} \ldots x_{n}\right]} \leq \operatorname{Var}_{n+1}\left(\frac{g_{\beta}}{\beta}\right)-\frac{g_{\beta}(x)}{\beta}
$$

usando que $\frac{g_{\beta}}{\beta}$ tem variação somável temos que para $n$ suficientemente grande a equação acima se transforma em

$$
-\epsilon-\frac{g_{\beta}(x)}{\beta} \leq \frac{1}{\beta} \log \frac{\mu_{\beta}\left[x_{1} \ldots x_{n}\right]}{\mu_{\beta}\left[x_{0} \ldots x_{n}\right]} \leq \epsilon-\frac{g_{\beta}(x)}{\beta},
$$

e como o limite

$$
\lim _{\beta \rightarrow \infty} \frac{1}{\beta} \log \frac{\mu_{\beta}\left[x_{1} \ldots x_{n}\right]}{\mu_{\beta}\left[x_{0} \ldots x_{n}\right]} \quad \text { existe para alguma subsequência de }\left(\beta_{i}\right),
$$

logo tomando o limite quando $\epsilon \rightarrow 0, \beta \rightarrow \infty$ e $n \rightarrow \infty$ na equação (4.25) obtemos

$$
R \leq \liminf _{n \rightarrow \infty} \lim _{\beta \rightarrow \infty} \frac{1}{\beta} \log \frac{\mu_{\beta}\left[x_{1} \ldots x_{n}\right]}{\mu_{\beta}\left[x_{0} \ldots x_{n}\right]} \leq R
$$

assim

$$
\liminf _{n \rightarrow \infty} \lim _{\beta \rightarrow \infty} \frac{1}{\beta} \log \frac{\mu_{\beta}\left[x_{1} \ldots x_{n}\right]}{\mu_{\beta}\left[x_{0} \ldots x_{n}\right]}=R(x) .
$$

Analogamente obtemos que

$$
\limsup _{n \rightarrow \infty} \lim _{\beta \rightarrow \infty} \frac{1}{\beta} \log \frac{\mu_{\beta}\left[x_{1} \ldots x_{n}\right]}{\mu_{\beta}\left[x_{0} \ldots x_{n}\right]}=R(x) .
$$

Portanto

$$
\lim _{n \rightarrow \infty} \lim _{\beta \rightarrow \infty} \frac{1}{\beta} \log \frac{\mu_{\beta}\left[x_{1} \ldots x_{n}\right]}{\mu_{\beta}\left[x_{0} \ldots x_{n}\right]}=R(x) .
$$


Corolário 5 (J. Mengue, [Men10]). Se há dois limites:

$$
\frac{g_{\beta_{i}}}{\beta_{i}} \rightarrow-R_{1} \quad e \frac{g_{\beta_{j}}}{\beta_{j}} \rightarrow-R_{2}
$$

com $R_{1} \neq R_{2}$. Então existem cilindros para os quais o limite

$$
\lim _{\beta \rightarrow \infty} \frac{1}{\beta} \log \left(\mu_{\beta}(C)\right)
$$

não existe.

Demonstração. Fixado $x \in \Sigma_{A}(\mathbb{N})$ e $\epsilon>0$ tal que $R_{1}(x)+\epsilon<R_{2}(x)$, pela proposição acima, existe $n$ suficientemente grande tal que

$$
\limsup _{\beta_{i} \rightarrow \infty} \frac{1}{\beta_{i}} \log \frac{\mu_{\beta_{i}}\left[x_{0} \ldots x_{n}\right]}{\mu_{\beta_{i}}\left[x_{1} \ldots x_{n}\right]}<R_{1}(x)+\frac{\epsilon}{2},
$$

$\mathrm{e}$

$$
\liminf _{\beta_{j} \rightarrow \infty} \frac{1}{\beta_{j}} \log \frac{\mu_{\beta_{j}}\left[x_{0} \ldots x_{n}\right]}{\mu_{\beta_{j}}\left[x_{1} \ldots x_{n}\right]}>R_{2}(x)-\frac{\epsilon}{2}>R_{1}(x)+\frac{\epsilon}{2} .
$$

Isto mostra que não existe para ambos cilindros

$$
\lim _{\beta \rightarrow \infty} \frac{1}{\beta} \log \mu_{\beta}\left[x_{0} \ldots x_{n}\right] \text { e } \lim _{\beta \rightarrow \infty} \frac{1}{\beta} \log \mu_{\beta}\left[x_{1} \ldots x_{n}\right] .
$$

Observação 8. Note que se $V_{1}$ e $V_{2}$ sao duas sub-ações calibradas diferentes, então definimos

$$
\begin{aligned}
& R_{1}(x)=f(x)+V_{1}(x)-V_{1} \circ \sigma(x)-m(f) \\
& R_{2}(x)=f(x)+V_{2}(x)-V_{2} \circ \sigma(x)-m(f)
\end{aligned}
$$

e usando a Proposição 7 segue que

$$
\begin{aligned}
V_{1}(x) & =V_{1}(a)+S_{f}(a, x) \\
V_{1}(\sigma(x)) & =V_{1}(a)+S_{f}(a, \sigma(x))
\end{aligned}
$$

$e$

$$
\begin{aligned}
V_{2}(x) & =V_{2}(a)+S_{f}(a, x) \\
V_{2}(\sigma(x)) & =V_{2}(a)+S_{f}(a, \sigma(x))
\end{aligned}
$$

assim

$$
V_{1}(x)-V_{1}(\sigma(x))=V_{2}(x)-V_{2}(\sigma(x)) .
$$

portanto se duas sub-ações diferem por uma constante concluímos que $R_{1}=R_{2}$.

Proposição 11. Se $\sum_{j=0}^{\infty} R\left(\sigma^{j}(x)\right)<\infty$ e $p \in \operatorname{supp}(\mu)$ então p é um ponto de acumulação de $\sigma^{n}(x)$.

Demonstração. Suponha que $\sum_{j=0}^{\infty} R\left(\sigma^{j}(x)\right)<\infty$, definimos para cada $n \in \mathbb{N}$ a medida de probabilidade

$$
\mu_{n}=\frac{1}{n} \sum_{j=0}^{n-1} \delta_{\sigma^{j}(x)} .
$$


Usando que $f$ é coerciva e $V$ é limitada obtemos que $-R=f+V-V \circ \sigma-m(f)$ é coerciva. E usando que $\sum_{j=0}^{\infty} R\left(\sigma^{j}(x)\right)<\infty$ concluímos que para $x=x_{0} x_{1} \ldots$ todos os símbolos $x_{i}$ pertencem a um alfabeto finito $B$. Isto mostra que $\mu_{n}$ tem o suporte contido em um conjunto compacto. Em particular existem medidas de acumulação na topologia fraca* para $\mu_{n}$.

Afirmação 5. Qualquer medida $\nu$ tal que $\lim _{n \rightarrow \infty} \mu_{n}=\nu$ é medida $f$-maximizante.

Demonstração. Seja $f_{k}:=\max (f,-k)$ é uma sequência de funções contínuas, limitadas e $f_{k} \searrow f$. Deste modo

$$
\begin{aligned}
\int f_{k} d \mu & =\lim _{n \rightarrow \infty} \frac{1}{n} \sum_{j=0}^{n-1} f_{k}\left(\sigma^{j}(x)\right) \\
& =\lim _{n \rightarrow \infty} \int f_{k} d \mu_{n} \\
& =\int f_{k} d \nu
\end{aligned}
$$

$\operatorname{logo} \lim _{k \rightarrow \infty} \int f_{k} d \nu=\lim _{k \rightarrow \infty} \int f_{k} d \mu$ e usando o teorema da convergência monótona obtemos que

$$
\int f d \nu=\int f d \mu=m(f)
$$

Assim a afirmação está provada.

Desta maneira concluímos que para qualquer ponto $p \in \operatorname{supp}(\mu)$ obtemos que

$$
\mu_{n}\left(\left\{x \in \Sigma_{A}(\mathbb{N}) ; d(x, p) \leq \epsilon\right\}\right)
$$

é positiva para $n$ suficientemente grande, portanto $p$ é um ponto de acumulação de $\sigma^{n}(x)$.

Observação 9. É natural supor que (ainda no caso da probabilidade maximizante não ser única) se existe o limite $\lim _{\beta \rightarrow \infty} \frac{1}{\beta} \log h_{\beta}=V$, então definindo $R=V \circ \sigma-V-f+m(f)$ (onde $\mu$ é qualquer medida maximizante) será possivel obter um princípio dos grandes desvios da forma da equação (4.1)? A resposta é não, A. T. Baraviera, A. O. Lopes, J. K. Mengue mostraram em [BLM13] que no caso de um shift de Bernoulli $X=\{0,1\}^{\mathbb{N}}$ para um potencial satisfazendo à condição de somabilidade de Walters tirando a hipótese da maximizante ser única, não é possivel obter um princípio dos grandes desvios da forma da equação (4.1).

Agora, provaremos que a função $\tilde{I}$ definida no Teorema 17 é igual à função taxa $I$ definida no Teorema 16:

\section{Proposição 12.}

$$
\tilde{I}(x)=\lim _{n \rightarrow \infty} \sum_{j=0}^{n-1} R\left(\sigma^{j}(x)\right) .
$$

Demonstração. Da Proposição 10 sabemos que

$$
\lim _{n \rightarrow \infty} \lim _{\beta_{i} \rightarrow \infty} \frac{1}{\beta_{i}} \log \frac{\mu_{\beta_{i}}\left[x_{1} \ldots x_{n}\right]}{\mu_{\beta_{i}}\left[x_{0} \ldots x_{n}\right]}=R(x),
$$

donde a sequência $\left(\beta_{i}\right)$ satisfaz (4.10). Assim a equação acima é equivalente a

$$
R=\lim _{n \rightarrow \infty}\left(\lim _{\beta_{i} \rightarrow \infty} \frac{1}{\beta_{i}} \log \mu_{\beta_{i}}\left[x_{1} \ldots x_{n}\right]-\lim _{\beta_{i} \rightarrow \infty} \frac{1}{\beta_{i}} \log \mu_{\beta_{i}}\left[x_{0} \ldots x_{n}\right]\right)
$$


portanto

$$
\tilde{I}(x)=R(x)+\tilde{I}(\sigma(x))
$$

Então para cada $n$

$$
\tilde{I}(x)=S_{n} R(x)+\tilde{I}\left(\sigma^{n}(x)\right),
$$

logo, fazendo $n \rightarrow \infty$ e usando o Teorema 17 parte 3 , obtemos

$$
\tilde{I}(x)=\sum_{j=0}^{\infty} R\left(\sigma^{j}(x)\right)+\tilde{I}_{0}(x) .
$$

Queremos provar que

$$
\tilde{I}(x)=\sum_{j=0}^{\infty} R\left(\sigma^{j}(x)\right) .
$$

Note que $\tilde{I}(x)=\sum_{j=0}^{\infty} R\left(\sigma^{j}(x)\right)+\tilde{I}_{0}(x) \geq \sum_{j=0}^{\infty} R\left(\sigma^{j}(x)\right)$. Por outro lado, se $\sum_{j=0}^{\infty} R\left(\sigma^{j}(x)\right)<\infty$, então qualquer $y \in \operatorname{supp}(\mu)$ pertence ao $\omega$-limite ${ }^{1}$ de $x$ (veja proposição 11). Seja $x=x_{0} x_{1} x_{2} \ldots$ fixo e $y \in \operatorname{supp}(\mu)$. Observe que, se $y \in \operatorname{supp}(\mu)$ então $\tilde{I}(y)=0$. Com efeito, para qualquer cilindro $C$ contendo o ponto limite temos que

$$
\lim _{\beta \rightarrow \infty} \frac{1}{\beta} \log \mu_{\beta}(C)=0 .
$$

Pois, da definição do suporte sabemos que $\mu(C)>0$ daí

$$
\lim _{\beta \rightarrow \infty} \log \mu_{\beta}(C)=\log \mu(C)=C T E,
$$

por conseguinte

$$
\tilde{I}(y)=-\lim _{n \rightarrow \infty} \lim _{\beta \rightarrow \infty} \frac{1}{\beta} \log \mu_{\beta}(C)=0 .
$$

Portanto, segue que

$$
\tilde{I}\left(x_{0} \ldots x_{n-1} y\right)=S_{n} R\left(x_{0} \ldots x_{n-1} y\right)+\tilde{I}(y)=S_{n} R\left(x_{0} \ldots x_{n-1} y\right) .
$$

Usando que $\tilde{I}$ é semi contínua inferiormente, temos que quando $n \rightarrow \infty$,

$$
\tilde{I}(x) \leq \liminf _{n \rightarrow \infty}\left(S_{n} R\left(x_{0} \ldots x_{n-1} y\right)\right) .
$$

Como $y$ pertence a $\omega$-limite de $x$ então fixado $m$, existe um $n$ tal que

$$
x_{n} x_{n+1} \ldots x_{n+m-1}=y_{0} y_{1} \ldots y_{m-1},
$$

e usando que $R$ tem variação somável, resulta que

$$
\begin{aligned}
\tilde{I}(x) & \leq S_{n} R\left(x_{0} \ldots x_{n-1} \ldots x_{n+m} y_{m} \ldots\right) \\
& \leq S_{n} R(x)+\operatorname{Var}_{m+n+1}(R)+\cdots+\operatorname{Var}_{m+1}(R) \\
& \leq \sum_{j=0}^{\infty} R\left(\sigma^{j}(x)\right)+\operatorname{Var}_{m+n+1}(R)+\cdots+\operatorname{Var}_{m+1}(R) .
\end{aligned}
$$

Assim fazendo $m \rightarrow \infty$ temos

$$
\tilde{I}(x) \leq \sum_{j=0}^{\infty} R\left(\sigma^{j}(x)\right) .
$$

\footnotetext{
${ }^{1} \omega(x)=\left\{y \mid \exists n_{i} \nearrow \infty \operatorname{com} \sigma^{n_{i}}(x) \rightarrow y\right\}$
} 
Portanto

$$
\tilde{I}(x)=\sum_{j=0}^{\infty} R\left(\sigma^{j}(x)\right)
$$

Deste modo, segue que para qualquer cilindro $C$ de $\Sigma_{A}(\mathbb{N})$, temos

$$
\lim _{\beta_{i} \rightarrow \infty} \frac{1}{\beta_{i}} \log \mu_{\beta_{i}}(C)=-\inf _{x \in C} \sum_{j=0}^{\infty} R\left(\sigma^{j}(x)\right) .
$$

Note que podemos repetir este processo para qualquer sequência $\left(\beta_{j}\right)$ tal que $\lim _{\beta_{j} \rightarrow \infty} \frac{1}{\beta_{j}} \log \mu_{\beta_{j}}$ exista, isto é, qualquer ponto de acumulação da sequência $\frac{1}{\beta_{j}} \log \mu_{\beta_{j}}$ é igual ao mesmo valor $-\inf _{x \in C} \sum_{j=0}^{\infty} R\left(\sigma^{j}(x)\right)$, em consequência

$$
\lim _{\beta \rightarrow \infty} \frac{1}{\beta} \log \mu_{\beta}(C)=-\inf _{x \in C} \sum_{j=0}^{\infty} R\left(\sigma^{j}(x)\right) .
$$

Usando algumas propriedades da Barreira de Peierls e o Potencial de Mañé encontramos a seguinte proposição que relaciona o Aubry e a função taxa $I$. De fato, já sabíamos que a função taxa $I$ se anulava nos pontos pertencentes ao $\operatorname{supp}(\mu)$. Veremos depois, na seção 3.2, que existe um conjunto denso de potenciais onde o $\operatorname{Aubry~é~o~} \operatorname{supp}(\mu)$ coincidem, assim para ditos potenciais vale que $I(x)=0$ se e somente se $x \in \operatorname{supp}(\mu)$.

Definimos

$$
\mathcal{R}:=\left\{f \mid \mathcal{M}_{\max }(f)=\{\mu\}, \Omega(f, \sigma)=\operatorname{supp}(\mu)\right\} .
$$

Proposição 13. Seja $\left(\Sigma_{A}(\mathbb{N}), \sigma\right)$ um shift finitamente primitivo. Seja $f: \Sigma_{A}(\mathbb{N}) \rightarrow \mathbb{R}$ com variação somável, $P(f)<\infty$ e $f \in \mathcal{R}$. E seja $V$ uma sub-ação calibrada limitada com variação somável, então:

$$
\Omega(f, \sigma)=\{x \mid I(x)=0\} .
$$

Demonstração. Vamos seguir as ideas de [CI99]. Sem perda de generalidade assumimos que $m(f)=0$. Primeiro provaremos que $\Omega(f, \sigma) \subset\{x \mid I(x)=0\}$. Como efeito, seja $x_{0} \in \Omega(f, \sigma)$ e $V$ uma sub-ação calibrada. Pela Proposição 6.(h) segue que $S_{f}\left(x_{0}, x_{0}\right)=0$, então existe $x_{k} \rightarrow x_{0}$, $n_{k} \uparrow \infty, \sigma^{n_{k}}\left(x_{k}\right)=x_{0}$ tal que $\lim _{k \rightarrow \infty} \sum_{j=0}^{n_{k}-1} f\left(\sigma^{j}\left(x_{k}\right)\right)=0$. Note também que

$$
\sum_{j=m+1}^{n_{k}+m-1} f\left(\sigma^{j}\left(x_{k}\right)\right)=\sum_{j=m+1}^{n_{k}+m-1}(f+V-V \circ \sigma)\left(\sigma^{j}\left(x_{k}\right)\right)-V\left(\sigma^{m+1}\left(x_{k}\right)\right)+V\left(\sigma^{m}\left(x_{0}\right)\right) .
$$

e

$$
\sum_{j=0}^{n_{k}-1} f\left(\sigma^{j}\left(x_{k}\right)\right)-\sum_{j=m+1}^{n_{k}+m-1} f\left(\sigma^{j}\left(x_{k}\right)\right)+f\left(\sigma^{m}\left(x_{0}\right)\right) \leq \sum_{j=0}^{m}\left|f\left(\sigma^{j}\left(x_{k}\right)\right)-f\left(\sigma^{j}\left(x_{0}\right)\right)\right| .
$$

Portanto usando equações (4.38) e (4.39) junto com o fato que que $f+V-V \circ \sigma \leq 0$ segue que 
para $m \in \mathbb{N}$

$$
\begin{aligned}
V\left(\sigma^{m+1}\left(x_{0}\right)\right) & \geq V\left(\sigma^{m}\left(x_{0}\right)\right)+f\left(\sigma^{m}\left(x_{0}\right)\right) \\
& \geq V\left(\sigma^{m+1}\left(x_{k}\right)\right)+\sum_{j=m+1}^{n_{k}+m-1} f\left(\sigma^{j}\left(x_{k}\right)\right)+f\left(\sigma^{m}\left(x_{0}\right)\right) \\
& \geq V\left(\sigma^{m+1}\left(x_{k}\right)\right)+\sum_{j=0}^{n_{k}-1} f\left(\sigma^{j}\left(x_{k}\right)\right)-\sum_{j=0}^{m}\left|f\left(\sigma^{j}\left(x_{k}\right)\right)-f\left(\sigma^{j}\left(x_{0}\right)\right)\right|
\end{aligned}
$$

Quando $k \rightarrow \infty$ o lado direito da equação (4.40) converge a $V\left(\sigma^{m+1}\left(x_{0}\right)\right)$, e aqui todas as desigualdades são igualdades. Deste modo, $R\left(\sigma^{m}\left(x_{0}\right)\right)=0$ para todo $m$ e aqui $I\left(x_{0}\right)=0$.

Provaremos agora a outra implicação. Com efeito, seja $x_{0} \in\{x \mid I(x)=0\}$. Pela Proposição 11 , existem $n_{k} \rightarrow \infty$ e $b \in \operatorname{supp} \mu$ tais que os limites $\lim _{k \rightarrow \infty} \sigma^{n_{k}}\left(x_{0}\right)=b \in \Sigma_{A}(\mathbb{N})$ e $\lim _{k \rightarrow \infty} \mu_{k}=\mu \in$ $\mathcal{M}_{\sigma}\left(\Sigma_{A}(\mathbb{N})\right)$ existem, aqui $\mu_{k}:=\frac{1}{n_{k}} \sum_{j=0}^{n_{k}-1} \delta_{\sigma^{j}\left(x_{0}\right)}$. Pela Proposição 6.(h), segue que $b \in \Omega(f, \sigma)$. Seja $V: \Sigma_{A}(\mathbb{N}) \rightarrow \mathbb{R}, V(x):=S_{f}(b, x)$, lembre que $V$ é uma sub-ação calibrada (veja a Proposição 6.(f)). Por conseguinte para $n_{k} \geq n$, segue que

$$
\begin{aligned}
V\left(\sigma^{n}\left(x_{0}\right)\right)+S_{f}\left(\sigma^{n}\left(x_{0}\right), \sigma^{n_{k}}\left(x_{0}\right)\right) & \geq V\left(\sigma^{n}\left(x_{0}\right)\right)+\sum_{j=n}^{n_{k}-1} f\left(\sigma^{j}\left(x_{0}\right)\right) \\
& =V\left(\sigma^{n_{k}}\left(x_{0}\right)\right) \quad\left(S_{f}(b, x)\right. \text { é uma sub-ação calibrada) } \\
& \geq V\left(\sigma^{n}\left(x_{0}\right)\right)+S_{f}\left(\sigma^{n}\left(x_{0}\right), \sigma^{n_{k}}\left(x_{0}\right)\right) \quad \text { (veja (3.30)). }
\end{aligned}
$$

Então todas as desigualdades são igualdades, portanto para $n_{k} \geq n$, temos

$$
S_{f}\left(\sigma^{n}\left(x_{0}\right), \sigma^{n_{k}}\left(x_{0}\right)\right)=\sum_{j=n}^{n_{k}-1} f\left(\sigma^{j}\left(x_{0}\right)\right) .
$$

E fazendo $n=0$ na equação (4.41) obtemos

$$
V\left(\sigma^{n_{k}}\left(x_{0}\right)\right)=V\left(x_{0}\right)+S_{f}\left(x_{0}, \sigma^{n_{k}}\left(x_{0}\right)\right)
$$

assim

$$
S_{f}\left(b, \sigma^{n_{k}}\left(x_{0}\right)\right)=S_{f}\left(b, x_{0}\right)+S_{f}\left(x_{0}, \sigma^{n_{k}}\left(x_{0}\right)\right)
$$

Pela Proposição 6.(j), tomando o limite em $k$ obtemos

$$
0=S_{f}(b, b)=S_{f}\left(b, x_{0}\right)+S_{f}\left(x_{0}, b\right)=0,
$$

desta forma

$$
0 \geq S_{f}\left(x_{0}, x_{0}\right) \geq S_{f}\left(x_{0}, b\right)+S_{f}\left(b, x_{0}\right)=0 .
$$

Portanto concluímos que $x_{0} \in \Omega(f, \sigma)$.

Corolário 6. Sob as mesmas hipóteses da proposição acima. Temos que:

1. Se $x \notin \Omega(f, \sigma)$ então $I(x)>0$.

2. Se $x \notin \Omega(f, \sigma)$ e $\sigma(x) \in \Omega(f, \sigma)$, então $R(x)>0$.

3. Para todo cilindro $C$ tal que $C \cap \operatorname{supp}(\mu)=\emptyset \Rightarrow \inf _{x \in C} I(x)>0$. 
Demonstração. 1. Pela proposição acima, sabemos que $\Omega(f, \sigma)=\{x \mid I(x)=0\}$. Como $I \geq 0$, isto prova item 1.

2. Já que $\sigma(x) \in \Omega(f, \sigma)$, resulta

$$
I(\sigma(x))=R(\sigma(x))+R\left(\sigma^{2}(x)\right)+\cdots=0,
$$

e como $x \notin \Omega(f, \sigma)$, por item $1, I(x)>0$, em consequência $R(x)>0$.

3. Suponha que existe um cilindro $C=\left[x_{0}, \ldots, x_{n}\right]$ tal que $C \cap \operatorname{supp}(\mu)=\emptyset \mathrm{e}$

$$
\inf _{x \in\left[x_{0} \ldots x_{n}\right]} I(x)=0 .
$$

Agora fixemos um ponto $x=x_{0} x_{1} \ldots$ fora do suporte e seja $z_{l} \in\left[x_{0} \ldots x_{n}\right]$, da equação (4.12) $\liminf _{l \rightarrow \infty} \inf _{z_{l} \in\left[x_{0} \ldots x_{n}\right]} I\left(z_{l}\right) \geq I(x)$, então usando (4.42) temos que $\inf _{z_{l} \in\left[x_{0} \ldots x_{n}\right]} I\left(z_{l}\right)=0$ portanto $I(x) \leq 0$ o qual é absurdo (veja item 1).

\subsection{Unicidade da medida maximizante}

Nesta seção construímos espaços de potenciais adequadas onde genericamente a medida maximizante é única e, a propriedade $\Omega(f, \sigma)=\operatorname{supp}(\mu)$ é densa. A construção é inspirada nas ideias de [DUZ07].

Como consequência do resultado de R. Bissacot e R. Freire [RJ14] temos o seguinte lema:

Lema 13. Seja $\left(\Sigma_{A}(\mathbb{N}), \sigma\right)$ um shift topologicamente transitivo, $\left(f_{k}\right)_{k}$ com variação somável e coercivo, $k \geq 1$. Seja $\left(\mu_{k}\right)_{k}$ uma sequência de medidas $f_{k}$-maximizante. Então existe um conjunto finito $\mathcal{A} \subset \mathbb{N}$ tais que $\operatorname{supp}\left(\mu_{k}\right) \subset \Sigma_{A}(\mathcal{A}), \forall k \geq 1$.

Observação 10. Dada uma sequência de medidas $\left(\mu_{k}\right)$ em $\mathcal{M}_{\sigma}\left(\Sigma_{A}(\mathbb{N})\right) \operatorname{com} \operatorname{supp}\left(\mu_{k}\right) \subseteq \Sigma_{A}(N)$, podemos construir uma nova sequência de medidas $\left(\lambda_{k}\right) \in \mathcal{M}_{\sigma}\left(\Sigma_{A}(N)\right)$. Com efeito, seja

$$
\lambda_{k}(B):=\mu_{k}\left(B \cap \operatorname{supp}\left(\mu_{k}\right)\right) \text { para todo conjunto de Borel } B \in \Sigma_{A}(N) .
$$

Portanto $\left(\lambda_{k}\right)$ tem subsequência convergente, digamos $\left(\lambda_{k_{j}}\right)$, isto é

$$
\lim _{j \rightarrow \infty} \lambda_{k_{j}}(B)=\lim _{j \rightarrow \infty} \mu_{k_{j}}\left(B \cap \operatorname{supp}\left(\mu_{k_{j}}\right)\right)=\lambda(B) .
$$

Em consequência $\left(\mu_{k}\right)$ tem uma subsequência convergente $\left(\lambda_{k_{j}}\right)$.

Lema 14. Sejam $\left(\Sigma_{A}(\mathbb{N}), \sigma\right)$ topologicamente transitivo e $f, f_{k}$ com variação somável e coercivos. Seja $\lim _{k \rightarrow \infty} f_{k}=f$ (com a norma do supremo), $\mu_{k}$ uma medida maximizante para $f_{k}$ e $\mu$ um ponto de acumulação para $\mu_{k}$, para todo $k \geq 1$, então

$$
\lim _{k \rightarrow \infty} m\left(f_{k}\right)=m(f)
$$

e $\mu$ é uma medida f-maximizante.

Demonstração. Seja $\mu$ uma medida de probabilidade $\sigma$-invariante qualquer, então

$$
\int f_{k} d \mu \leq m\left(f_{k}\right)
$$

por hipóteses, $\left\|f_{k}-f\right\|_{\infty} \rightarrow 0$, então

$$
\int f d \mu \leq \liminf _{k \rightarrow \infty} m\left(f_{k}\right),
$$


portanto

$$
m(f) \leq \liminf _{k \rightarrow \infty} m\left(f_{k}\right)
$$

Por outro lado, temos que

$$
\begin{aligned}
m\left(f_{k}\right)=\int f_{k} d \mu_{k} & =\int\left(f_{k}-f\right) d \mu_{k}+\int f d \mu_{k} \\
& \leq\left\|f_{k}-f\right\|_{\infty}+m(f),
\end{aligned}
$$

assim

$$
\limsup _{k \rightarrow \infty} m\left(f_{k}\right) \leq m(f) .
$$

Por conseguinte

$$
\lim _{k \rightarrow \infty} m\left(f_{k}\right)=m(f) .
$$

Agora como $\mu$ é um ponto de acumulação para $\left(\mu_{k}\right)$ segue que $\lim _{k \rightarrow \infty} \int f_{k} d \mu_{k}=\int f d \mu$, assim concluímos que $\mu$ é uma medida $f$-maximizante.

Observação 11. Lembre que o espaço $U C B\left(\Sigma_{A}(\mathbb{N})\right)$ é um espaço de Banach (veja Corolário 7 ), ademais as funçôes dependendo de um número finito de coordenadas são densas em $\operatorname{UCB}\left(\Sigma_{A}(\mathbb{N})\right)$ (veja Lema 16). Ainda mais, se escolhemos um conjunto de funçôes dependo de um número finito de coordenadas com valores racionais, segue que $U C B\left(\Sigma_{A}(\mathbb{N})\right)$ é um espaço de Banach separável (veja Lema 17). Considere agora $Y$ um espaço métrico fechado qualquer de $U C B\left(\Sigma_{A}(\mathbb{N})\right)$, assim $Y$ é um espaço métrico separável e Banach. Considere o espaço

$$
X:=f+Y=\{h=f+g ; g \in Y\} .
$$

Como $Y$ é um espaço métrico separável de Banach então $X$ é um espaço métrico separável de Banach, também.

Teorema 18. Sob as hipóteses do Lema 14 genericamente em X existe uma única medida maximizante.

Demonstração. Seja $\left(\psi_{n}\right)$ uma sequência densa em $X$. Duas medidas diferentes, digamos $\mu$ e $\nu \in$ $\mathcal{M}_{\max }(f)$, devem dar valores diferentes para alguma $\psi_{n}$. Isto é

$$
\begin{aligned}
\left\{f \in X: \mathcal{M}_{\max }(f)>1\right\} & =\left\{f \in X: \exists n \exists \mu, \nu \in \mathcal{M}_{\max }(f), \int \psi_{n} d \nu \neq \int \psi_{n} d \mu\right\} \\
& =\bigcup_{n}\left\{f \in X: \exists \mu, \nu \in \mathcal{M}_{\max }(f), \int \psi_{n} d \nu \neq \int \psi_{n} d \mu\right\} \\
& =\bigcup_{n} \bigcup_{m}\left\{f \in X: \exists \mu, \nu \in \mathcal{M}_{\max }(f),\left|\int \psi_{n} d \nu-\int \psi_{n} d \mu\right| \geq \frac{1}{m}\right\} .
\end{aligned}
$$

Definimos

$$
F_{n, m}:=\left\{f \in X: \exists \mu, \nu \in \mathcal{M}_{\max }(f),\left|\int \psi_{n} d \nu-\int \psi_{n} d \mu\right| \geq \frac{1}{m}\right\} .
$$

Agora, mostraremos que o conjunto $F_{n, m}$ é fechado em $X$. Com efeito, considere uma sequência $\left(f_{k}\right)$, com $P\left(f_{k}\right)<\infty, k \geq 1$ tais que $f_{k} \rightarrow f$ (com a norma do supremo), já que $f_{k} \in F_{n, m}$, existem duas seqüências de medidas $f_{k}$-maximizantes, $\left(\mu_{k}\right)$ e $\left(\nu_{k}\right)$ cumprindo

$$
\left|\int \psi_{n} d \mu_{k}-\int \psi_{n} d \nu_{k}\right| \geq \frac{1}{m}
$$


Escolhemos $\left(\mu_{k_{j}}\right)$ subsequência de $\left(\mu_{k}\right)$ e $\left(\nu_{k_{j}}\right)$ subsequência de $\left(\nu_{k}\right)$, com limites $\mu$ e $\nu$ respectivamente (veja Lema 13 e Observação 10). Do Lema 14 sabemos que, $\mu$ e $\nu$, são $f$-maximizantes. Ademais satisfazem

$$
\left|\int \psi_{n} d \mu-\int \psi_{n} d \nu\right| \geq \frac{1}{m}
$$

Portanto $f \in F_{n, m}$, assim $F_{n, m}$ é fechado.

Agora, mostraremos que os conjuntos $F_{n, m}$ tem interior vazio. Fixando $f$, afirmamos que a função $\varepsilon \rightarrow m\left(f+\varepsilon \psi_{n}\right)$ é convexa mas não diferenciável em $\varepsilon=0$. Com efeito, assuma $\mu$ e $\nu$ são $f$-maximizantes e

$$
\int \psi_{n} d \mu \geq \int \psi_{n} d \nu+\frac{1}{m}
$$

então, a derivada direita é maior que $\int \psi_{n} d \mu$, e a derivada esquerda é menor que $\int \psi_{n} d \nu$. É sabido que uma função convexa é diferenciável Lebesgue quase em toda parte (em realidade quase em toda parte excepto em um conjunto enumerável), isto prova que existem infinitos $\varepsilon$ se acumulando em 0 tal que $f+\varepsilon \psi_{n}$ não pode estar em $F_{n, m}$. Por conseguinte $F_{n, m}$ tem interior vazio, portanto $F_{n, m}$ é um conjunto magro. Lembre do Teorema de Baire, que o complementar de um magro é um conjunto denso.

Definimos $\mathcal{F}$ como o espaço das funções com variação somável satisfazendo a seguinte condição:

$$
\forall K \subset \Sigma_{A}(\mathbb{N}) \text { compacto } \exists \psi \in C^{0} \text { tal que } \psi \leq 0,\{x \mid \psi(x)=0\}=K .
$$

Proposição 14. Seja $\left(\Sigma_{A}(\mathbb{N}), \sigma\right)$ um shift finitamente primitivo. Seja $f: \Sigma_{A}(\mathbb{N}) \rightarrow \mathbb{R}$ com variação somável e $P(f)<\infty$, então

$$
\mathcal{R}:=\left\{f \in \mathcal{F} \mid \mathcal{M}_{\max }(f)=\{\mu\}, \Omega(f, \sigma)=\operatorname{supp}(\mu)\right\}
$$

é um conjunto denso em $X$.

Demonstração. O conjunto $\mathcal{D}:=\left\{f \mid \mathcal{M}_{\max }(f)=\{\mu\}\right\}$ é denso em $X$ (veja Teorema 18). Dado $f \in$ $\mathcal{D}$ com $\mathcal{M}_{\max }(f)=\{\mu\}$ e $\epsilon>0$, seja $\psi \in C^{0}$ tal que $\|\psi\|_{0}<\epsilon, \psi \leq 0$ e $\{x \mid \psi(x)=0\}=\operatorname{supp}(\mu)$. Observe que $\mathcal{M}_{\max }(f)=\mathcal{M}_{\max }(f+\psi)$. Com efeito, como $\psi \leq 0$, então $\mathcal{M}_{\max }(f+\psi) \leq \mathcal{M}_{\max }(f)$. Por outro lado note que

$$
\mathcal{M}_{\max }(f)=\int_{\operatorname{supp}(\mu)} f d \mu=\int_{\operatorname{supp}(\mu)}(f+\psi) d \mu \leq \mathcal{M}_{\max }(f+\psi) .
$$

Seja $x_{0} \notin \operatorname{supp}(\mu)$. Dado $\delta>0$, e $z \in \Sigma_{A}(\mathbb{N})$ tal que $\sigma^{n}(z)=x_{0}$ e $d\left(x_{0}, z\right)<\delta$, então

$$
\begin{aligned}
\sum_{j=0}^{n-1}(f+\psi)\left(\sigma^{j}(z)\right) & \leq S_{f}^{\delta}\left(x_{0}, x_{0}\right)+\sum_{j=0}^{n-1} \psi\left(\sigma^{j}(z)\right) \\
& \leq S_{f}^{\delta}\left(x_{0}, x_{0}\right)+\psi(z) .
\end{aligned}
$$

Tomando o supremo sobre todos os $n \geq 1$ e $z$ tais que $\sigma^{n}(z)=x_{0}$ e $d\left(x_{0}, z\right)<\delta$, e fazendo $\delta \rightarrow 0$, obtemos

$$
S_{f+\psi}\left(x_{0}, x_{0}\right) \leq S_{f}\left(x_{0}, x_{0}\right)+\psi\left(x_{0}\right) \leq \psi\left(x_{0}\right)<0 .
$$

Portanto $x_{0} \notin \Omega(f+\psi, \sigma)$. Pela proposição 6.(h), sabemos que $\operatorname{supp}(\mu) \subset \Omega(f+\psi, \sigma)$, assim concluímos que $\Omega(f+\psi, \sigma)=\operatorname{supp}(\mu)$ e portanto $f+\psi \in \mathcal{R}$.

Assim provamos que, dado $f \in \mathcal{D}$ e $\epsilon>0 \exists f+\psi \in \mathcal{R}$ tal que $\|f-(f+\psi)\|_{0}=\|\psi\|_{0}<\epsilon$. Em consequência $\mathcal{D} \subset \overline{\mathcal{R}} \operatorname{logo} \overline{\mathcal{D}} \subset \overline{\overline{\mathcal{R}}}=\overline{\mathcal{R}}$ então $\mathcal{R}$ é um conjunto denso em $X$. 


\subsection{Pesquisas futuras}

Dados os resultados até aqui, as seguintes questões são naturais:

1. Estender os resultados obtidos na tese para o caso de um shift BIP e potencial Walters. Nesta linha o que falta é analiticidade da pressão, já que todos os cálculos feitos nesta tese passariam também para o caso Walters assumindo a analiticidade da pressão. Em [CS09] O. Sarig prova que a analiticidade da pressão está ligada ao fato que o potencial tenha a propriedade do gap espectral. Mais precisamente, ele encontra condições suficientes e necessárias para que o operador de Ruelle de um potencial localmente Hölder contínuo sobre um shift de Markov topologicamente mixing atue com gap espectral sobre algum espaço de Banach. Como Y. Daon já estendeu boa parte dos resultados para shifts transitivos e com potencias Walters em [Dao12], é natural esperar que essa propriedade também se estenda.

2. O caso topologicamente mixing e não BIP está em aberto e provavelmente será necessário novas ideias. As dificuldades deste caso são: a automedida não é de probabilidade e autofunção não está uniformemente afastada de zero e de infinito.

Teorema 19 (O. Sarig, [Sar01]). Seja $\Sigma_{A}(\mathbb{N})$ um Renewal shift e f localmente Hölder continua tais que $\sup f<\infty$. Então existe uma constante $\beta_{c} \in(0, \infty]$ tais que

- Para $0<\beta<\beta_{c}$ existe uma probabilidade de equilíbrio $\mu_{\beta}$ corresponde a $\beta f$. Para $\beta>\beta_{c}$ não existe medida de equilíbrio correspondente a $\beta f$.

- $P(\beta f)$ é analítica real em $\left(0, \beta_{c}\right)$ e linear em $\left(\beta_{c}, \infty\right)$. Em $\beta_{c}, P(\beta f)$ é contínua mas não analítica.

Teorema 20 (G. Iommi, [Iom07]). Seja $\Sigma_{A}(\mathbb{N})$ um Renewal shift e $f$ localmente Hölder continua com $\sup f<\infty$. Então

- Para $\beta_{c}=\infty$, então existe uma medida $f$-maximizante.

- Se $\beta_{c}<\infty$, então não existe medida $f$-maximizante.

Combinando esse resultado com o trabalho de R. Bissacot e R. Freire (veja o Teorema 13), onde eles provam existência de medidas maximizantes para o caso de um shift transitivo e potencial de variação somável e coercivo, (Renewal é um exemplo de um shift topologicamente mixing e portanto transitivo), concluímos que a pressão é sempre analítica. Portanto é de esperar que neste caso também exista um princípio dos grandes desvios.

3. Provar um princípio dos grandes desvios tirando a hipótese da medida maximizante ser única. Nesta linha a função taxa não seria a a mesma da forma da equação (4.2), pois A. T. Baraviera, A. O. Lopes, J. K. Mengue mostraram em [BLM13] que no caso de um shift de Bernoulli $X=\{0,1\}^{\mathbb{N}}$ para um potencial satisfazendo a condição de somabilidade de Walters tirando a hipótese da maximizante ser única, não é possível obter um princípio dos grandes desvios como a mesma função taxa. 


\section{Apêndice A}

\section{Espaços de Potenciais:caso não-compacto}

O espaço $C_{b}\left(\Sigma_{A}(\mathbb{N})\right)$ de funções contínuas e limitadas de valor real será equipada com a norma uniforme $d(\varphi, \psi)=\sup _{x \in \Sigma_{A}(\mathbb{N})}|(\varphi-\psi)(x)|$, qual torna ele num espaço de Banach. Seja $U C B\left(\Sigma_{A}(\mathbb{N})\right)$ denota o espaço de funções uniformemente contínuas e limitadas de valor real. Esse é um subespaço fechado de $C_{b}\left(\Sigma_{A}(\mathbb{N})\right)$, então ele mesmo é um espaço de Banach equipado com norma uniforme.

Lema 15. $U C B\left(\Sigma_{A}(\mathbb{N})\right)$ é um subespaço fechado de $C_{b}\left(\Sigma_{A}(\mathbb{N})\right)$.

Demonstração. Seja $f_{n}$ uma sequência de funções em $\operatorname{UCB}\left(\Sigma_{A}(\mathbb{N})\right)$ qual converge a $f$ (ou seja $\left.\left\|f_{n}-f\right\|_{\infty} \rightarrow 0\right)$.

Mostraremos que $f$ é (uniformemente) contínua, isto é, dado $\epsilon>0$ existe $\delta>0$ tal que $\mid f(x)-$ $f\left(x^{\prime}\right) \mid<\epsilon$ se $d\left(x, x^{\prime}\right)<\delta$.

Com efeito, seja $\epsilon>0$ dado e seja $N$ tal que $\left\|f_{n}-f\right\|_{\infty}<\epsilon / 4$ para todo $n \geq N$, isto significa $\left|f_{n}(t)-f(t)\right|<\epsilon / 4$ para todo $t \in \Sigma_{A}(\mathbb{N})$ e todo $n \geq N$.

Como $f_{N}$ é (uniformemente) contínua existe $\delta>0$ tal que $\left|f_{N}(x)-f_{N}\left(x^{\prime}\right)\right|<\epsilon / 2$ sempre que $d\left(x, x^{\prime}\right)<\delta$.

Para $x, x^{\prime}$ temos também que

$$
\begin{aligned}
\left|f(x)-f\left(x^{\prime}\right)\right| & \leq\left|f(x)-f_{N}(x)\right|+\left|f_{N}(x)-f_{N}\left(x^{\prime}\right)\right|+\left|f_{N}\left(x^{\prime}\right)-f\left(x^{\prime}\right)\right| \\
& \leq 2\left\|f-f_{N}\right\|_{\infty}+\left|f_{N}(x)-f_{N}\left(x^{\prime}\right)\right|<2 \epsilon / 4+\epsilon / 2=\epsilon .
\end{aligned}
$$

Corolário 7. $U C B\left(\Sigma_{A}(\mathbb{N})\right)$ é um espaço de Banach.

Demonstração. Lembre que $C_{b}\left(\Sigma_{A}(\mathbb{N})\right)$ é um espaço de Banach.

Lema 16. As funções dependendo de finitas coordenadas são densas em $\operatorname{UCB}\left(\Sigma_{A}(\mathbb{N})\right)$.

Demonstração. Seja $f$ em $\operatorname{UCB}\left(\Sigma_{A}(\mathbb{N})\right)$, provaremos que, existe $\left(f_{n}\right)$ dependendo de finitas coordenadas tal que $f_{n} \rightarrow f$ (com a norma uniforme). Com efeito

Seja $x \in \Sigma_{A}(\mathbb{N})$ qualquer, como $f \in U C B\left(\Sigma_{A}(\mathbb{N})\right)$, sabemos que dado $\epsilon>0, \exists \delta>0$ tal que

$$
|f(x)-f(z)|<\epsilon \text { sempre que } d(x, z)<\delta .
$$

Agora, para cada $n \geq 1$, defina $f_{n}(x):=\sup _{x \in\left[x_{0} \ldots x_{n-1}\right]} f(x)$. Assim usando (A.1) concluímos que dado $\epsilon>0$ existe $n$ suficientemente grande tais que

$$
\left|f_{n}(x)-f(x)\right|<\epsilon
$$

Lema 17. $U C B\left(\Sigma_{A}(\mathbb{N})\right)$ é um espaço de Banach separável. 
Demonstração. Do Corolário 7 sabemos que $U C B\left(\Sigma_{A}(\mathbb{N})\right)$ é um espaço de Banach; e do Lema 16 as funções dependendo de finitas coordenadas são densas em $U C B\left(\Sigma_{A}(\mathbb{N})\right)$, assim para concluir que $U C B\left(\Sigma_{A}(\mathbb{N})\right)$ é separável é suficiente tomar um conjunto de funções dependendo de finitas coordenadas assumindo valores racionais.

Lema 18. Seja $B V\left(\Sigma_{A}(\mathbb{N})\right)=\left\{g: \Sigma_{A}(\mathbb{N}) \rightarrow \mathbb{R}, \sum_{n=0}^{\infty} \operatorname{Var}_{n}(g)<\infty\right\}$ é um espaço separável e Banach.

Demonstração. Vejamos que $B V\left(\Sigma_{A}(\mathbb{N})\right)$ é Banach com a norma $\|\cdot\|$ dada por

$$
\|g\|=\|g\|_{\infty}+\sum_{k=1}^{\infty} \operatorname{Var}_{k}(g) .
$$

Com efeito, Seja $\left(f_{n}\right)_{n}$ uma sequência de cauchy em $B V$. Então dado $\epsilon>0 \exists N \in \mathbb{N}$ tal que

$$
\left\|f_{n}-f_{m}\right\|=\left\|f_{n}-f_{m}\right\|_{\infty}+\sum_{k=1}^{\infty} \operatorname{Var}_{k}\left(f_{n}-f_{m}\right)<\epsilon \quad \forall m, n \geq N .
$$

Assim que $\left\|f_{n}-f_{m}\right\|_{\infty} \leq\left\|f_{n}-f_{m}\right\|<\epsilon \forall n, m \geq N$ então $\left(f_{n}\right)_{n}$ é uma sequência de cauchy em $\|\cdot\|_{\infty}$, assim existe $f \in U C B\left(\Sigma_{A}(\mathbb{N})\right)$ tais que $f_{n} \rightarrow f$ (uniformemente).

\section{Afirmação 6.}

(i) Se $\lim _{n \rightarrow \infty} \sup _{x}\left|\left(f_{n}-f\right)(x)\right|=0$ então $\lim _{n \rightarrow \infty} \operatorname{Var}_{k}\left(f_{n}-f\right)=0$. (Em particular $\lim _{n \rightarrow \infty} \operatorname{Var}_{k}\left(f_{n}\right)=$ $\left.\operatorname{Var}_{k}(f)\right)$.

(ii) $\left\|f_{n}-f\right\| \rightarrow 0$.

(iii) $f \in B V\left(\Sigma_{A}(\mathbb{N})\right)$.

Com efeito,

(i)

$$
\begin{aligned}
\operatorname{Var}_{k}\left(f_{n}-f\right) & =\sup \left\{\left|\left(f_{n}-f\right)(x)-\left(f_{n}-f\right)(y)\right| ; x_{0}=y_{0}, \ldots, x_{k-1}=y_{k-1}\right\} \\
& \leq \sup _{x}\left|\left(f_{n}-f\right)(x)\right|+\sup _{y}\left|\left(f_{n}-f\right)(y)\right| \leq 2\left\|f_{n}-f\right\|_{\infty} \rightarrow 0 .
\end{aligned}
$$

(ii) Usando (A.2) sabemos que

$$
\sum_{k=1}^{\infty} \operatorname{Var}_{k}\left(f_{n}-f_{m}\right)<\epsilon \forall n, m \geq N
$$

assim

$$
\liminf _{n \rightarrow \infty} \sum_{k=1}^{\infty} \operatorname{Var}_{k}\left(f_{n}-f_{m}\right)<\epsilon \forall n, m \geq N
$$

Para $m$ fixo, definimos $f_{n}(k):=\operatorname{Var}_{k}\left(f_{n}-f_{m}\right)$, e note que $\sum_{k=1}^{\infty} f_{n}(k)=\int f_{n}(k) d \mu(k)$, onde $\mu$ é a medida de contagem sobre $\mathbb{N}$. Assim usando o lema de Fatou sabemos $\int \liminf _{n \rightarrow \infty} f_{n}(k) d \mu(k) \leq$ $\liminf _{n \rightarrow \infty} \int f_{n}(k) d \mu(k)$. Portanto temos que

$$
\sum_{k=1}^{\infty} \liminf _{n \rightarrow \infty} \operatorname{Var}_{k}\left(f_{n}-f_{m}\right) \leq \liminf _{n \rightarrow \infty} \sum_{k=1}^{\infty} \operatorname{Var}_{k}\left(f_{n}-f_{m}\right)<\epsilon \forall n, m \geq N
$$


Assim usando o item $(i)$ resulta

$$
\sum_{k=1}^{\infty} \lim _{n \rightarrow \infty} \operatorname{Var}_{k}\left(f_{n}-f_{m}\right)<\epsilon \forall n, m \geq N
$$

assim

$$
\sum_{k=1}^{\infty} \operatorname{Var}_{k}\left(f_{m}-f\right)<\epsilon \quad \forall m \geq N
$$

(iii) Aqui $\operatorname{Var}(f) \leq \operatorname{Var}\left(f_{n}-f\right)+\operatorname{Var}\left(f_{n}\right)$. Seja $N$ tais que $\operatorname{Var}\left(f_{m}-f_{n}\right) \leq 1$ se $m, n \geq N$. Assim para cada $n$

$$
\operatorname{Var}\left(f_{n}\right) \leq \max \left\{1+\operatorname{Var}\left(f_{N}\right), \operatorname{Var}\left(f_{1}\right), \ldots, \operatorname{Var}\left(f_{N-1}\right)\right\}
$$

e usando o item (ii) concluímos que $\operatorname{Var}(f)<\infty$.

Similarmente à prova do Lema 16 podemos verificar que a sequência $f_{n}(x):=\sup _{x \in\left[x_{0} \ldots x_{n-1}\right]} f(x)$ é densa em $B V\left(\Sigma_{A}(\mathbb{N})\right)$.

Seja $X$ um espaço topológico separável. $f: X \rightarrow \mathbb{R}$. Para cada subconjunto compacto $K$ de $X$, podemos definir a seminorma $\|\cdot\|_{C^{0}(K)}$ sobre $C^{0}(K)$ por $\|f\|_{C^{0}(K)}:=\sup _{x \in K}|f(x)|$. A topologia gerada por todas estas seminormas é chamada a topologia de convergência em compactos (ou algumas vezes "topologia de convergência uniforme sobre conjuntos compactos"). Aqui as "bolas"deste espaço $\left(C^{0}(X),\|\cdot\|_{C^{0}(K)}\right)$ estão dadas por

$$
B_{K}(f, \epsilon):=\left\{g\left|\sup _{x \in K}\right| f(x)-g(x) \mid<\epsilon\right\} .
$$

A razão para a escolha desta terminologia vem do seguinte teorema, cuja prova é imediata.

Teorema 21. Uma sequência $f_{n} \in C^{0}(X)$ converge a $f \in C^{0}(X)$ na topologia de convergência em compactos se e somente se para cada conjunto compacto $K$ de $X$, a sequência $\left.f_{n}\right|_{K}$ converge uniformemente a $\left.f\right|_{K}$.

Proposição 15. Seja $X$ um espaço topológico separável, e seja $\left(f_{n}\right)$ uma sequência equicontínua de $C^{0}(X)$. Assuma que existe alguma $f \in C^{0}(X)$ e algum subconjunto denso $D$ de $X$ tal que $\lim _{n \rightarrow \infty} f_{n}(x)=f(x)$ para cada $x \in D$. Entẫo $\left(f_{n}\right)$ converge uniformemente a $f$ em qualquer subconjunto compacto $K \subset X$.

Demonstração. Seja $\epsilon>0$. Pela eqüicontinuidade de $\left(f_{n}\right)$ e a continuidade de $f$, para cada $x \in X$, existe alguma vizinhança $V_{x}$ de $x$ tal que $\left|f_{n}(y)-f_{n}(x)\right|<\epsilon$ para todo $y \in V_{x}$ e todo $n$; e

$|f(y)-f(x)|<\epsilon$ para todo $y \in V_{x}$.

Pela compacidade de $K$, existem $z_{1}, \ldots, z_{k} \in K$ tal que $K \subset \bigcup_{i=1}^{k} V_{z_{i}}$. Agora, seja $y \in V_{z_{i}}$. Escolher $x \in D \cap V_{z_{i}}$, e algum $m_{i}$ com

$\left|f_{n}(x)-f(x)\right|<\epsilon$ para todo $n>m_{i}$. Note que

$$
|f(x)-f(y)| \leq\left|f(x)-f\left(z_{i}\right)\right|+\left|f\left(z_{i}\right)-f(y)\right|<2 \epsilon .
$$

Desta maneira

$$
\begin{aligned}
& \left|f_{n}(y)-f(y)\right| \\
& \leq\left|f_{n}(y)-f_{n}\left(z_{i}\right)\right|+\left|f_{n}\left(z_{i}\right)-f_{n}(x)\right|+\left|f_{n}(x)-f(x)\right|+|f(x)-f(y)| \\
& <5 \epsilon
\end{aligned}
$$


para todo $y \in V_{z_{i}}$ e $n>m_{i}$.

Finalmente, colocar $m=\max \left\{m_{i}: 1 \leq i \leq k\right\}$, e notar que $\left|f_{n}(y)-f(y)\right|<5 \epsilon$ para todo $y \in K$ e todo $n>m$. 


\section{Apêndice B}

\section{Grandes Desvios na Probabilidade}

Um principio dos grandes desvios caracteriza o comportamento limite, quando $n \rightarrow \infty$ de uma familia de medidas de probabilidades $\left(\mu_{n}\right)_{n}$ em termos de uma função taxa:

Definição 26. Seja $\left(\mu_{n}\right)_{n}$ uma sequência de medidas de probabilidade sobre os subconjuntos de Borel $\mathcal{B}\left(\Sigma_{A}\right)$ de $\Sigma_{A}$. A função $I: \Sigma_{A} \rightarrow[0, \infty]$ é chamada função taxa se:

1. I é semicontinua inferiormente.

2. Para cada número real $b>0$ o conjunto $\left\{x \in \Sigma_{A}: I(x) \leq b\right\}$ é um subconjunto fechado de $\Sigma_{A}$.

Definição 27. A sequência $\left(\mu_{n}\right)_{n}$ satisfaz um Princípio dos Grandes Desvios com função taxa I(·) se:

1. Para cada conjunto aberto $O$ de $\Sigma_{A}$

$$
\liminf _{n \rightarrow \infty} \frac{1}{n} \log \mu_{n}(O) \geq-\inf _{x \in O} I(x),
$$

2. Para cada conjunto fechado $G$ de $\Sigma_{A}$

$$
\limsup _{n \rightarrow \infty} \frac{1}{n} \log \mu_{n}(G) \leq-\inf _{x \in G} I(x) .
$$

Agora, para qualquer sequência $\left(\mu_{n}\right)$ cumprindo a definição de acima temos

$$
-\inf _{x \in B^{\circ}} I(x) \leq \liminf _{n \rightarrow \infty} \frac{1}{n} \log \mu_{n}(B) \leq \limsup _{n \rightarrow \infty} \frac{1}{n} \log \mu_{n}(B) \leq-\inf _{x \in \bar{B}} I(x)
$$

para cada conjunto de Borel $B \subseteq \Sigma_{A}$.

Em particular

$$
\lim _{n \rightarrow \infty} \frac{1}{n} \log \mu_{n}(B)=-\inf _{x \in B} I(x)
$$

sempre que $\inf _{x \in B^{\circ}} I(x)=\inf _{x \in \bar{B}} I(x)$.

Unicidade de função taxa

Sejam $I_{1}$ e $I_{2}$ satisfazendo a Definição 27 e seja $x \in \Sigma_{A}$. Usando a equação (B.2) para $I_{1}$ e $(B .1)$ para $I_{2}$ obtemos:

$$
\begin{aligned}
-I_{2}(x) \leq-\inf _{y \in B_{\delta}(x)} I_{2}(y) & \leq \liminf _{n \rightarrow \infty} \frac{1}{n} \log \mu_{n}\left(B_{\delta}(x)\right) \\
& \leq \limsup _{n \rightarrow \infty} \frac{1}{n} \log \mu_{n}\left(\bar{B}_{\delta}(x)\right) \leq \inf _{y \in \bar{B}_{\delta}(x)} I_{1}(y) .
\end{aligned}
$$


Observe que, não é difícil verificar que a semi-continuidade de $I$ implica que o termo da direita tende a $-I_{1}(x)$ quando $\delta \downarrow 0$, assim $I_{1}(x) \leq I_{2}(x)$. Revertendo os papéis de $I_{1}$ e $I_{2}$, obtemos $I_{1}=I_{2}$. 


\section{Referências Bibliográficas}

$\left[\mathrm{BCL}^{+} 11\right]$ A. Baraviera, L. Cioletti, A. O. Lopes, J. Mohr e R. Souza. On the general onedimensional XY model: positive and zero temperature, selection and non-selection. Reviews in Mathematical Physics., 23:1063-1113, 2011. 19

[BLL12] A. Baraviera, R. Leplaideur e A. O. Lopes. Selection of ground states in the zerotemperature limit for a one-parameter family of potentials. SIAM J. Appl. Dyn. Syst, $11: 243-260,2012$.

[BLL13] A. Baraviera, R. Leplaideur e A. O. Lopes. Ergodic Optimization, zero temperature limits and the max-plus algebra. 2013. 2, 23, 35

[BLM13] A. Baraviera, A. O. Lopes e J. K. Mengue. On the selection of subaction and measure for a subclass of potentials defined by P. Walters. Ergod. Th. and Dynam. Sys., 33:13381362, 2013. 57, 64

[BLT06] A. Baraviera, A. O. Lopes e P. Thieullen. A large deviation principle for the equilibrium states of Holder potentials:the zero temperature case. Stochastics and Dynamics, 6:7796, 2006. 2,21

[Bou01] T. Bousch. La condition de Walters. Ann. Sci. Éc. Norm Supér, 34:287-311, 2001. 2, 26

[Bow08] R. Bowen. Equilibrium States and Ergodic Theory of Anosov Diffeomorphisms. Lect. Notes in Math. Springer-Verlag, 2nd ed edição, 2008. 1, 9

[Bré03] J. Brémont. Gibbs measures at temperature zero. Nonlinearity, 16:419-426, 2003. 19

[BS03] J. Buzzi e O. Sarig. Uniqueness of equilibrium measures for countable Markov shifts and multidimensional piecewise expanding maps. Ergod. Th. and Dynam. Sys., 23:1383-1400, 2003. 1,9

[Car09] T. Cardoso. Alguns resultados sobre otimização ergódica em espaços não compactos. Dissertação de Mestrado, Universidade de São Paulo, 2009.

[CGU11] J. R. Chazottes, J. M. Gambaudo e E. Ugalde. Zero-temperature limit of one-dimensional gibbs states via renormalization: the case of locally constant potentials. Ergod. Th. and Dynam. Sys., 31:1109-1161, 2011. 19

[CH10] J. R. Chazottes e M. Hochman. On the zero-temperature limit of Gibbs states. Comm. Math. Phys., 297:265-281, 2010. 19

[CI99] G. Contreras e R. Iturriaga. Global minimizers of autonomous Lagrangians. 1999. 32, 59

[CLO13] G. Contreras, A. O. Lopes e E. R. Oliviera. Ergodic Transport Theory, Periodic Maximizing Probabilities and the Twist Condition. Springer, 2013. 35 
[CLT01] G. Contreras, A. O. Lopes e P. Thieullen. Lyapunov minimizing measures for expanding maps of the circle. Ergod. Th. and Dynam. Sys., 21:1379-1409, 2001. 2, 23, 32, 35, 45

[Con14] G. Contreras. Ground states are generically a periodic orbit. Pré-publicação, 2014. 23

[CR14] D. Coronel e J. Rivera. Sensitive dependence of Gibbs measures. Pré-publicação, 2014. 19

[CS09] V. Cyr e O. Sarig. Spectral gap and transience for Ruelle operators on countable Markov shifts. Commun. Math. Phys, 292:637-666, 2009. 64

[Dao12] Y. Daon. Bernoullicity of equilibrium measures on countable Markov shifts. Prépublicação, 2012. 1, 9, 64

[DUZ07] A. M. Davie, M. Urbański e A. Zdunik. Maximizing measures on metrizable non- compact spaces. Proceedings of the Edinburgh Mathematical Society, 50:123-151, 2007. 2, 61

[DZ98] A. Dembo e O. Zeitouni. Large deviations techniques and applications. Springer-Verlag, 1998. 49

[El106] R. S. Ellis. Entropy, large deviations, and Statistical Mechanics. Springer, 2006. 49

[FFY02] D. Fiebig, U. R. Fiebig e M. Yuri. Pressure and equilibrium states for countable state Markov shifts. Israel Journal of Mathematics, 131:221-257, 2002.

[Gar06] E. Garibaldi. Otimização Ergódica: da maximização relativa aos homeomorfismos expansivos. Tese de Doutorado, Universidade Federal do Rio Grande do Sul, 2006. 25, 35

[Gar09] E. Garibaldi. Introduction to expanding Ergodic Optimization. 2009. 35

[Geo88] H. O. Georgi. Gibbs measures and phase transitions. Walter de Gruyter, Berlin-New york, 1988.

[GL08] E. Garibaldi e A. O. Lopes. On the Aubry-Mather theory for symbolic dynamics. Ergod. Th. and Dynam. Sys., 28:791-815, 2008. 32, 35

[GLT09] E. Garibaldi, A. O. Lopes e P. Thieullen. On calibrated and separating sub-actions. Bull. Braz. Math. Soc., 40:577-602, 2009. 32, 35

[IJ13] G. Iommi e T. Jordan. Phase transitions for suspension flows. Commun. Math. Phys, $320: 475-498,2013.2$

[Iom07] G. Iommi. Ergodic Optimization for Renewal type shifts. Monatsh. Math., 150:91-95, 2007. $2,8,64$

[Jen06] O. Jenkinson. Ergodic Optimization. Discrete contin. Dyn. Syst. Ser A, 15:197-224, 2006. $1,2,23$

[JMU05] O. Jenkinson, R. D. Mauldin e M. Urbański. Zero temperature limits of Gibbsequilibrium states for countable alphabet subshifts of finite type. Journal of Statistical Physics, 119:765-776, 2005. 2, 19, 25, 31

[JMU06] O. Jenkinson, R. D. Mauldin e M. Urbański. Ergodic Optimization for countable alphabet subshifts of finite type. Ergod. Th. and Dynam. Sys., 26:1791-1803, 2006. 2, 20, 23, $24,25,31,41$

[JMU07] O. Jenkinson, R. D. Mauldin e M. Urbański. Ergodic Optimization for non-compact dynamical systems. Dynamical Systems, 22:379-388, 2007. 23, 25 
[Kem11a] T. Kempton. Thermodynamic Formalism for symbolic dynamical systems. Tese de Doutorado, The University of Warwick, 2011.

[Kem11b] T. Kempton. Zero temperature limits of Gibbs equilibrium states for countable Markov shifts. Journal of Statistical Physics, 143:795-806, 2011. 19

[LC14] A. O. Lopes e L. Cioletti. Interactions, specifications, DLR probabilities and the Ruelle operator in the one-dimensional lattice. Pré-publicação, 2014. 12

[Lep05] R. Leplaideur. A dynamical proof for the convergence of Gibbs measures at temperature zero. Nonlinearity, 18:2847-2880, 2005. 19

[LM10] A. O. Lopes e J. K. Mengue. Zeta measures and Thermodynamic Formalism for temperature zero. Bull. Braz. Math. Soc, 41:449-480, 2010.

[LM14] A. O. Lopes e J. K. Mengue. Selection of measure and a large deviation principle for the general one-dimensional XY model. Dynamical Systems., 29:24-39, 2014.

[LMST09] A. O. Lopes, J. Mohr, R. Souza e P. Thieullen. Negative entropy, pressure and zero temperature: a l.d.p. for stationary Markov chains on $[0,1]$. Bull. Braz. Math. Soc., 40:1-52, 2009.

[Mañ96] R. Mañé. Generic properties and problems of minimizing measures of Lagragian systems. Nonlinearity, 9:273-310, 1996. 32

[Men10] J. K. Mengue. Zeta medidas e princípio dos grandes desvios. Tese de Doutorado, Universidade Federal do Rio Grande do Sul, 2010. 56

[Mor] I. D. Morris. Entropy for zero-temperature limits of Gibbs equilibrium states for countable alphabet subshifts of finite type. Journal of Statistical Physics, 126:315-324. 2, 20

[Mor07] I. D. Morris. A sufficient condition for the subordination principle in Ergodic Optimization. Bulletin of the London Mathematical Society, 39:214-220, 2007. 25

[MU01] D. Mauldin e M. Urbański. Gibbs states on the symbolic space over an infinite alphabet. Israel Journal of Mathematics, 125:93-130, 2001. 11

[MU03] R. D. Mauldin e M. Urbański. Graph directed Markov systems: geometry and dynamics of limit sets. Cambridge University Press, 2003. 1, 11

[OV04] E. Olivieri e M. E. Vares. Large deviations and metastability. Cambridge University Press, 2004. 49

[Pes14] Y. Pesin. On the work of Sarig on countable Markov chains and Thermodynamic Formalism. Journal of Modern Dynamics, 8:1-14, 2014. 1

[PP90] W. Parry e M. Pollicott. Zeta functions and the periodic orbit structure of hyperbolic dynamics. Astérisque, 1990.

[QS12] A. Quas e J. Siefken. Ergodic Optimization of super-continuous functions. Ergodic Theory Dynamical Systems, 32:2071-2082, 2012. 2, 23

[RG10] R.Bissacot e E. Garibaldi. Weak KAM methods and ergodic optimal problems for countable Markov shifts. Bull. Braz. Math. Soc, 41:321-338, 2010. 20, 23, 25, 26, 41

[RJ14] R.Bissacot e R. Freire Jr. On the existence of maximizing measure for irreducible countable Markov shift: a dynamical proof. Ergod. Th. and Dynam. Sys, 34:1103-1115, 2014. $20,23,25,41,61$ 
[Rue68] D. Ruelle. Statistical Mechanics of a one-dimensional lattice gas. Commun. Math. Phys, 9:267-278, 1968.

[Rue78] D. Ruelle. Thermodynamic Formalism. Encyclopedia of Mathematics and its Applications. Addison-Wesley, 1978. 1, 9

[Sar99] O. Sarig. Thermodynamic Formalism for countable Markov shift. Ergodic Theory and Dynamical Systems, 19:1565-1593, 1999. 1, 9, 10, 12

[Sar00] O. Sarig. On an example with topological pressure which is not analytic. C. R. Acad.Sci. Paris série I, 330:311-315, 2000. 1

[Sar01] O. Sarig. Phase transitions for countable Markov shifts. Commun. Math. Phys, 217:555$577,2001.1,8,19,64$

[Sar03] O. Sarig. Existence of Gibbs measures for countable Markov shifts. Proc. of AMS, 131:1751-1758, 2003. 1, 2, 12

[Sar09] O. Sarig. Lectures notes on Thermodynamic Formalism for topological Markov shifts. 2009. $1,9,10,12$

[TB02] O. Jenkinson T. Bousch. Cohomology classes of dynamically non-negative $c^{k}$ functions. Invent. math, páginas 207-217, 2002.

[vEW07] A. C. D. van Enter e W. M. Ruszel. Chaotic temperature dependence at zero temperature. J. Stat. Phys, 127:567-573, 2007. 19

[VJ67] D. Vere-Jones. Ergodic properties of nonnegative matrices -I. Pac. J. Math, 22:361-385, 1967. 8

[VJ15] V. A. Vargas e R. Freire Jr. Equilibrium states and zero temperature limit for summable Markov potentials on topologically mixing shifts. Pré-publicação, 2015. 11, 19

[Wal78] P. Walters. Invariant measures and equilibrium states for some mappings which expand distances. Trans. American Math. Soc, 236, 1978. 6

[Wal82] P. Walters. An introduction to Ergodic Theory. Springer-Verlag, 1982. 9, 13, 14, 50 